\title{
Semigroups of Linear Operators in a Banach Space
}

By

\author{
Shinnosuke OHARU*
}

This paper concerns the construction of the solution of an abstract Cauchy problem and the generation of semigroups of bounded linear operators in a Banach space.

Let $A$ be a closed linear operator in a Banach space $X$ and let us consider a differential equation $(d / d t) u(t)=A u(t)$ in $X$. Our first problem is to find the solution of this equation associated with the given initial value $u(0)=x$, under some additional conditions on $A$. The additional conditions on $A$ are stated roughly as follows:

(1) The resolvent set $\rho(A)$ of $A$ contains a half real line $(\omega, \infty)$; and hence for each nonnegative integer $n, D\left(A^{n}\right)$.can be regarded as a Banach space with respect to the graph norm; this is condition $(I ; \omega)$.

(2) There is a nonnegative integer $k$ such that the operators $\xi^{n} R(\xi$; $A)^{n}, \xi$ large and $n=1,2,3, \ldots$, map bounded sets in the Banach space $D\left(A^{k}\right)$ into bounded sets in $X$, where $R(\xi ; A)$ denotes the resolvent of $A$ at $\xi$; this is the idea behind condition $(I I ; k)$ or $\left(I I_{\exp } ; k\right)$ mentioned later.

Then, under these conditions there is a one-parameter family $\left\{U_{t}\right.$; $t \geqq 0\}$ of continuous linear operators from a Banach space $D\left(A^{m}\right)$ into $X$ and the family gives a unique solution operator of the Cauchy problem for $A$, where $m=2 k+1$ in general and $m=k$ if $A$ is densely defined. The proof given in this paper is based on that of the author [17]. We can also apply other methods which are analogous to Kato [9], Feller [6]

Received January 12, 1971.

Communicated by K. Yosida.

* Department of Mathematics, Waseda University, Tokyo, Japan. 
and Crandall-Liggett [2]. Hille-Yosida-Miyadera-Phillips' theorem states that a linear operator $A$ is the infinitesimal generator of a $\left(C_{0}\right)$-semigroup if and only if $A$ is a densely defined, closed linear operator satisfying $(I ; \omega)$ and $(I I ; 0)$; the corresponding $\left\{U_{t} ; t \geqq 0\right\}$ becomes the $\left(C_{0}\right)$-semigroup. In this sense the above result is an extension of the generation theorem of $\left(C_{0}\right)$-semigroups.

The solution operator $\left\{U_{t}\right\}$ is closely related to the notion of distribution semigroup. For instance, a linear operator $A$ is the infinitesimal generator of an exponential distribution semigroup if and only if $A$ is a densely defined, closed linear operator satisfying conditions $(I ; \omega)$ and $\left(I I_{\text {exp }} ; k\right)$ for some $\omega$ and $k$. Also, $\left\{U_{t}\right\}$ can be regarded as an $R$ semigroup which was studied by $\mathrm{Da}$ Prato [4]. We shall discuss some relationships among $\left\{U_{t}\right\}$ and these notions of semigroups. The results obtained will give some informations on the continuity at the origin $t=0$ and the regularity of exponential distribution semigroups.

The solution operator $\left\{U_{t}\right\}$ mentioned above can not necessarily be extended to a semigroup of bounded linear operators. In fact, in order to extend such a solution operator to a semigroup of bounded linear operators, it is required that $A$ be densely defined and that the solution of the Cauchy problem for $A$ depend continuously on initial data. Krein considered in $[11]$ the semigroup obtained by extending the solution operator, under the assumption that the problem be correctly posed. The condition of correct posedness is discussed by $\operatorname{Lax}$ [13] or [21] and it is in fact equivalent to a Feller type condition which is suggested by Hille-Phillips $[8 ;$ p. 373$]$, see also Feller $[6]$. In view of this, we obtain the following result:

If $A$ is a densely defined, closed linear operator in a Banach space $X$ satisfying conditions $(I ; \omega)$ and $(I I ; k)$ for some $\omega$ and $k$ (which guarantee the existence of a solution operator for a Cauchy problem formulated for $A$ ) and a condition of Feller type, then we obtain a semigroup $\left\{T_{t} ; t>0\right\}$ of bounded linear operators such that

$$
T_{t} x=\lim _{h \rightarrow+0}(I-h A)^{-[t / h]} x, \quad x \in D\left(A^{k}\right),
$$

where the convergence is uniform with respect to $t$ in every finite inter- 
val;

(ii) for every $x \in D\left(A^{k+1}\right), T_{t} x$ gives the solution of the abstract Cauchy problem for $A$ associated with the initial value $x$;

(iii) $D\left(A^{k}\right) \subset \Sigma$, where $\Sigma$ is the continuity set which is defined by $\Sigma=\left\{x \in X ; \lim _{t \rightarrow+0} T_{t} x=x\right\}$.

Our second problem is to extend well-known classes stated in HillePhillips $[8 ; \S 10.6]$ and study the fundamental structure of such semigroups. For this purpose, it is natural to classify the semigroups of bounded linear operators obtained as above in terms of the continuity set $\Sigma$. That is, for each nonnegative integer $k$, we consider a class of semigroups $\left\{T_{t}\right.$; $t>0\}$ such that $D\left(A^{k}\right) \subset \Sigma$, where $A$ is the closure of its infinitesimal generator. In this paper, such a class will be called class $\left(C_{(k)}\right)$. We shall characterize these classes.

The characterization of the semigroup of class $\left(C_{(k)}\right)$ given in this paper is in substance an extension of that of the semigroup of class $\left(C_{0}\right)$. However, it is another purpose to study the relationships among the classes $\left(C_{(k)}\right), k=0,1,2,3, \ldots$, and various well-known notions of semigroups. First, it can be shown that every $\left(C_{(k)}\right)$-semigroup can be extended to an exponential distribution semigroup. Conversely, every semigroup which can be extended to a regular distribution semigroup belongs to some class $\left(C_{(k)}\right)$. Class $\left(C_{(0)}\right)$ is the same thing as class $\left(C_{0}\right)$; class $(0, A)$ is an important subclass of class $\left(C_{(1)}\right)$; and class $(A)$ is a particular case of class $\left(C_{(2)}\right)$.

Finally, we shall discuss that the theory of semigroups of continuous linear operators in a locally convex space can be employed to construct semigroups of classes $\left(C_{(k)}\right)$.

In the present paper we restrict ourselves to the case in which the infinitesimal generator of the semigroups treated has a non-empty resolvent set. As for the case in which the resolvent set of the infinitesimal generator is empty, we shall publish it elsewhere.

Section 1 deals with the basic notions and some of their properties.

Section 2 concerns the abstract Cauchy problem on a finite interval.

Section 3 concerns the construction of the semigroup solution of an 
abstract Cauchy problem in a Fréchet space.

Section 4 contains some results on the abstract Cauchy problem on a real half line.

Section 5 deals with some relations among the results of Section 4 and the notion of distribution semigroup. Also, in that section, we discuss a characterization of the semigroup of bounded linear operators which can be extended to a distribution semigroup.

Finally, Section 6 gives a characterization of class $\left(C_{(k)}\right)$. Also, in that section some generation theorems of well-known classes will be obtained.

The author wants to express his deep gratitude to Professor I. Miyadera, Professor H. Sunouchi, Mr. N. Okazawa and Mr. T. Ushijima for their many valuable suggestions.

\section{Preliminaries}

In this section, we introduce some basic notions and notations which will be used in this paper.

Let $X$ and $Y$ be (complex) Banach spaces. Let $A$ be a linear operator (or simply an operator) from $X$ into $Y$. We denote by $D(A)(\subset X)$ and $R(A)(\subset Y)$ the domain and range of $A$, respectively. We write $\mathbb{C}(X, Y)$ for the totality of closed operators $A$ with $D(A) \subset X$ and $R(A) \subset Y$. Also, we write $\mathfrak{B}(X, Y)$ for the totality of bounded operators on $X$ into $Y$. However, for brevity in notation, we write $\mathfrak{S}(X)$ and $\mathfrak{B}(X)$ for $\mathfrak{S}(X, X)$ and $\mathfrak{B}(X, X)$ respectively. Similarly, when $X$ and $Y$ are locally convex spaces, we write $\mathscr{L}(X, Y)$ and $\mathcal{L}(X)$ for the totality of continuous operators on $X$ into $Y$ and that of continuous operators on $X$ into itself, respectively.

Let $A$ be an operator from $X$ into itself, then we say simply that $A$ is an operator in $X ; \rho(A)$ denotes the resolvent set of $A$ and for $\lambda \in \rho(A)$ we assume that $R(\lambda ; A)$ means the resolvent of $A$ at $\lambda$. Let $A$ be an operator from $X$ into itself, then we mean by $N(A)$ the null space of $A$. If $N(\lambda-A)=\{0\}$, then $(\lambda-A)^{-1}$ is defined as an operator from $Y$ into $X$; we use the notation $J_{\lambda}$ which stands for $(I-\lambda A)^{-1}$, when $A$ is fixed 
and $N\left(\lambda^{-1}-A\right)=\{0\}$.

Let $S \subset X$, then $\bar{S}$ means the closure of $S$. Accordingly, we denote by $\bar{A}$ the closure of a closable operator $A$. Let $S \subset X$ and $A$ be an operator from $X$ into $Y$, then we write $A \mid S$ for the restriction of $A$ to $D(A)$ $\cap S$. For any closable operator $A$ such that $\bar{A}=B$, its domain $D(A)$ is called a core of $B$. In other words, a linear manifold $D(\subset D(B))$ is a core of $B$, if $D$ is dense in $D(B)$ with respect to the graph norm of $B$.

We use the following abbreviations: Let $X_{1}$ be a linear manifold in $X$ and $A$ be an operator from $X$ into $Y$. When we consider $A$ as an operator from $X_{1}$ into $Y$ (i.e., $A \mid X_{1}$ ), we say simply that $A$ is an operator from $X_{1}$ into $Y$. Accordingly, if $X_{1}$ is a Banach space with respect to a certain norm, then $A \in \mathfrak{B}\left(X_{1}, Y\right)$ means that $A \mid X_{1} \in \mathfrak{B}\left(X_{1}, Y\right)$.

Now, assume that $A \in \mathfrak{S}(X)$, then we may regard $D\left(A^{n}\right)$ as a Banach space with respect to the norm $\|x\|+\|A x\|+\cdots+\left\|A^{n} x\right\|$; we write $\|x\|_{n}$ for the norm and $\left[D\left(A^{n}\right)\right]$ for the Banach space. We note that if $A, A^{2}$, $\ldots, A^{n} \in \mathfrak{S}(X)$ then the above-mentioned norm $\|x\|_{n}$ is equivalent to the graph norm of $A^{n}$.

Let $U \in \mathfrak{B}\left(\left[D\left(A^{n}\right)\right], X\right)$, then we denote by $\|U\|_{n}$ for the operator norm of $U$. We shall abbreviate $\mathfrak{B}\left(\left[D\left(A^{n}\right)\right], X\right)$ by $\mathfrak{B}\left(D\left(A^{n}\right), X\right)$. Also, in this paper, we let $A^{0}=I ; I$ denotes the identity operator and we assume that $\left[D\left(A^{0}\right)\right]=X$. Let $U \in \mathfrak{B}(X)$, then $U \in \mathfrak{B}\left(D\left(A^{n}\right), X\right)$; we shall write $\|U\|_{n}$ for $\left\|U \mid D\left(A_{n}\right)\right\|_{n}$, for brevity in notation.

Throughout this paper, we write $\mathbf{R}=(-\infty, \infty), \mathbf{R}_{+}=(0, \infty), \overline{\mathbf{R}}_{+}=$ $[0, \infty)$, and $\mathbf{Z}_{+}$for the totality of nonnegative integers.

Let $X$ be a Banach space. We write $\lim x_{n}=x$ or $x_{n} \rightarrow x$ as $n \rightarrow \infty$, if a sequence $\left\{x_{n}\right\} \subset X$ converges to some $x \in X$ strongly. Let $\left\{U_{n}\right\} \subset \mathfrak{B}$ $(X)$. We then write $s$-lim $U_{n}=U$, if $\left\{U_{n}\right\}$ converges to some $U \in \mathfrak{B}(X)$ in the sense of the strong operator topology.

Now, we introduce the notion of an (abstract) Cauchy problem, $A C P$. Let $X$ be a Banach space and $A$ be an operator in $X$, and then let us consider the differential equation

$$
(d / d t) u(t)=A u(t)
$$

where $(d / d t)$ means the differentiation in the sense of the strong topology. 
In this setting, we formulate the following problem:

$A C P$. Given a positive number $T$ and an element $x \in X$, find a function $u(t) \equiv u(t ; x)$ such that

(i) $u(t)$ is strongly absolutely continuous and strongly continuously differentiable in $[0, T]$ (or $(0, T])$;

(ii) for each $t \in(0, T], u(t) \in D(A)$ and $u(t)$ satisfies (1.1);

(iii) $\lim _{t \rightarrow+0} u(t)=x$.

This problem is called the (abstract) Cauchy problem, ACP, formula ted for an operator $A$ on $[0, T]$ and the $X$-valued function $u(t ; x)$ satisfying (i), (ii) and (iii) is called the solution associated with the initial value $x$. There are two alternatives in condition (i); the corresponding problems will be denoted by $A C P_{1}$ and $A C P_{2}$ respectively. Similarly, we can formulate $A C P_{i}, i=1,2$, for an operator $A$ on $[0, \infty)$ (on $(0, \infty)$ ); the solution $u(t)$ of $A C P_{1}$ for $A$ on $[0, \infty)$ (resp. $A C P_{2}$ for $A$ on $(0, \infty)$ ) is that of $A C P_{1}$ for $A$ on $(0, T]$ (resp. $A C P_{2}$ for $A$ on $(0, T]$ ) if $u(t)$ is restricted to a finite interval $[0, T]$ (resp. $(0, T]$ ).

In the following, we state some notions of semigroups of operators in a Banach space $X$.

A one-parameter family $\left\{T_{t} ; t>0\right\} \subset \mathfrak{B}(X)$ is called a semigroup (of bounded operators), if it has the following properties:

$$
\begin{array}{ll}
T_{t+s}=T_{t} T_{s}, & t, s>0, \\
s-\lim _{t \rightarrow t_{0}} T_{t}=T_{t_{0}}, & t_{0}>0 .
\end{array}
$$

We define the infinitesimal generator $A_{0}$ by $A_{0} x=\lim _{h \rightarrow+0} A_{h} x, A_{h}=h^{-1}$ $\left[T_{h}-I\right]$, whenever the limit exists, and the type $\omega_{0}$ by $\omega_{0}=\lim _{t \rightarrow+\infty} t^{-1}$ $\log \left\|T_{t}\right\|_{\text {. }}$

The $\omega_{0}$ is always defined and $\omega_{0}<+\infty$, see Hille-Phillips [8; Theorem 7.6.1]. Also, according to Feller [6], we call the set $\Sigma=$ $\left\{x \in X ; \lim _{t \rightarrow+0} T_{t} x=x\right\}$ the continuity set of $\left\{T_{t}\right\}$. We define

$$
R_{0}(\lambda) x=\int_{0}^{\infty} e^{-\lambda t} T_{t} x d t
$$

for $\lambda \in \mathbb{C}$ and $x \in X$, whenever the integral makes sense. It is easily seen 
that $D\left(R_{0}(\lambda)\right) \supset \Sigma$ provided that $\operatorname{Re}(\lambda)>\omega_{0}$.

Here, we state some definitions of well-known classes of semigroups. A semigroup $\left\{T_{t}\right\}$ is said to be of class $(A)$, if $X_{0}=\bigcup T_{t>0}[X]$ is dense in $X$ and if there exists an $\omega_{1}>\omega_{0}$ such that for each $\lambda$ with $\operatorname{Re}(\lambda)>\omega_{1}$, there exists an $R(\lambda) \in \mathfrak{B}(X)$ with the properties

$$
\begin{aligned}
& R(\lambda) x=R_{0}(\lambda) x, \quad \text { for } x \in X_{0} ; \\
& \sup \left\{\|R(\lambda)\| ; \operatorname{Re}(\lambda)<\omega_{1}\right\}<+\infty ; \\
& s-\lim _{\lambda \rightarrow+\infty} \lambda R(\lambda)=I .
\end{aligned}
$$

If furthermore,

$$
\int_{0}^{1}\left\|_{1} T_{t} x\right\| d t<+\infty, \text { for } x \in X \quad \text { or }(\mathrm{A} .4)^{\prime} \int_{0}^{1}\left\|T_{t}\right\| d t<+\infty,
$$

then such a semigroup $\left\{T_{t}\right\}$ is said to be of class $(0, A)$ or $(1, A)$ respectively. The infinitesimal generator $A_{0}$ of a semigroup of either class is densely defined and closable; $A=\bar{A}_{0}$ is called the complete infinitesimal generator. If $A$ is the complete infinitesimal generator of an $(A)$-semigroup, then $\left\{\lambda ; \operatorname{Re}(\lambda)>\omega_{1}\right\} C \rho(A)$ and $R(\lambda)=R(\lambda ; A)$ for $\operatorname{Re}(\lambda)>\omega_{1}$. If $\left\{T_{t}\right\}$ is a $(0, A)$ - or $(1, A)$-semigroup, then we can take $\omega_{1}=\omega_{0}$ and the relation (A.1) holds for all $x \in X$. Finally, a semigroup $\left\{T_{t}\right\}$ is said to be of class $\left(C_{0}\right)$, if $\Sigma=X$. For details, see Hille-Phillips $[8 ; \S 10.6]$.

$\mathrm{J}$. Lions introduced in [12] the notion of distribution semigroup. Let $D(\mathbf{R})$ and $D\left(\mathbf{R}_{+}\right)$be the Schwartz spaces corresponding to $\mathbf{R}$ and $\mathbf{R}_{+}$ respectively. Let $D^{\prime}(\mathfrak{B}(X))=\mathfrak{L}(D(\mathbf{R}), \mathfrak{B}(X))$ be the class of $\mathfrak{B}(X)$-valued distributions and $D_{+}^{\prime}(\mathfrak{B}(X))$ be the subclass of $D^{\prime}(\mathfrak{B}(X))$ which consists of the elements whose supports are contained in $\overline{\mathbf{R}}_{+}$. A $\mathfrak{B}(X)$-valued distribution $T \in D_{+}^{\prime}(\mathfrak{B}(X))$ is called a regular distribution semigroup (R.D.S.G.) on a Banach space $X$, if the following conditions are satisfied:

$$
T(\phi * \psi)=T(\phi) T(\psi) \quad \text { for } \phi, \phi \in D\left(\mathbf{R}_{+}\right)
$$

(D.2) $\bigcap_{\phi \in D\left(\mathbf{R}_{+}\right)} N(T(\phi))=\{0\} \quad$ and $\Re=\operatorname{sp}\left[\bigcup_{\phi \in D\left(\mathbf{R}_{+}\right)} R(T(\phi))\right]$ is dense in $X$;

(D.3) for every $x \in \Re$, there is an $X$-valued function $x(t)$ such that 
a) $x(t)=0$ for $t<0$ and $x(0)=x$,

b) $\quad x(t)$ is strongly continuous in $t \geqq 0$,

c) $T(\phi) x=\int_{0}^{\infty} \phi(t) x(t) d t \quad$ for $\phi \in D\left(\mathbb{R}_{+}\right)$.

If furthermore,

(D.4) there exists a real number $\omega$ such that $e^{-\lambda t} T$ is a tempered distribution (i.e., $e^{-\lambda t} T \in \mathfrak{S}^{\prime}(\mathfrak{B}(X))$ for every $\lambda>\omega$,

then $T$ is called an exponential distribution semigroup (E.D.S.G.).

Let $T$ be an R.D.S.G. For each $\mathbb{R}$-valued distribution $F$ with compact support contained in $\overline{\mathbb{R}}_{+}$, we can define a uniquely determined, densely defined and closable operator in $X$, denoted $T(F)$, by the relation

$$
T(F) x=\sum_{j=1}^{n} T\left(F * \phi_{j}\right) y_{j} \quad \text { for } x=\sum_{j=1}^{n} T\left(\phi_{j}\right) y_{j} \in \Re,
$$

see Peetre [19]. $\overline{T\left(-\delta^{\prime}\right)}$ is called the infinitesimal generator of $T$.

Da Prato $[3,4]$ extended the notion of semigroup of bounded operators and introduced the notion of $R$-semigroup. A one-parameter family $\left\{H_{t} ; t \geqq 0\right\} \subset \mathfrak{B}(X)$ is called an $R$-semigroup, if

$$
\begin{aligned}
& H_{t} H_{s}=H_{s} H_{t}=H_{t+s} H_{0} \quad \text { for } t, s \in \overline{\mathbf{R}}_{+}, \\
& N\left(H_{0}\right)=\{0\} \text { and } \overline{R\left(H_{0}^{n}\right)}=X \text { for } n \in \mathbb{Z}_{+}, \\
& H_{t} x \text { is strongly continuous in } t \geqq 0 \text { for } x \in X .
\end{aligned}
$$

If furthermore, there exist numbers $M>0$ and $\omega \in \mathbb{R}$ such that

$$
\left\|H_{t}\right\| \leqq M e^{\omega t} \quad \text { for } t \in \overline{\mathbf{R}}_{+} \text {, }
$$

then we say that $\left\{H_{t}\right\}$ is of exponential growth.

For instance, let $T$ be an E.D.S.G., $A$ the infinitesimal generator, and $p(\xi)$ be a polynomial of degree $n$ with nonnegative coefficients such that $\|R(\lambda ; A)\| \leqq p(|\lambda|)$ for $\operatorname{Re}(\lambda)>\omega$ and some $\omega \in \mathbf{R}$, then it is proved that $H_{t} \equiv T\left(\delta_{t}\right) R\left(\lambda_{0} ; A\right)^{n+2}, t \geqq 0$, define an $R$-semigroup, where $\operatorname{Re}\left(\lambda_{0}\right)>\omega$ and $\delta_{t}$ denotes the point mass concentrated at $t$. Let $D_{0}=\left\{x \in X ; \exists H_{0}^{\prime} x=\lim _{h \rightarrow+0}\right.$ 
$\left.h^{-1}\left[H_{h}-H_{0}\right] x\right\}$, then the infinitesimal generator $A_{0}$ of $\left\{H_{t}\right\}$ is defined by the relation

$$
A_{0} x=H_{0}^{-1} H_{0}^{\prime} x \quad \text { for } x \in D\left(A_{0}\right)=\left\{x \in D_{0} ; H_{0}^{\prime} x \in R\left(H_{0}\right)\right\} \text {. }
$$

It is proved [3] that $A_{0}$ is closable. Da Prato introduced the notion of generalized resolvent of $A$ and gave in [4] a characterization of an $R$ semigroup in terms of the generalized resolvent.

Finally, we state the notion of a locally equicontinuous semigroup which was recently studied by $\mathrm{T}$. Kōmura in [10]. Let $Y$ be a locally convex linear topological space. Then a one-parameter family $\left\{T_{t} ; t \geqq 0\right\}$ $\mathcal{C}(Y)$ is called a locally equicontinuous semigroup, if

$$
T_{0}=I, \quad T_{t} T_{s}=T_{t+s} \quad \text { for } t, s \geqq 0,
$$

(L.2) for every $y \in Y, T_{t} y$ is strongly continuous in $t \geqq 0$,

(L.3) for every continuous seminorm $p$ on $Y$ and $T>0$, there exists a continuous seminorm $q$ on $Y$ such that $p\left(T_{t} y\right) \leqq q(y)$ for $t \in[0, T]$ and $y \in Y$.

It is proved that if $Y$ is tonnelé, then every semigroup $\left\{T_{t} ; t \geqq 0\right\}$ $(C \&(Y)$ ) satisfying (L.2) is a locally equicontinuous semigroup. The infinitesimal generator is defined by $A x=\lim _{h \rightarrow+0} A_{h} x$ in $Y, A_{h}=h^{-1}\left[T_{h}-I\right]$, whenever the limit exists in the strong topology. Let $\left\{T_{t}\right\}$ be a semigroup of continuous operators on a locally convex, sequentially complete space $Y$ for which conditions (L.1) and (L.2) hold, then the infinitesimal generator is densely defined in $Y$. Also, the infinitesimal generator of a locally equicontinuous semigroup in a locally convex space is closed. For details, see Kōmura [10].

\section{Construction of the Solution of $A C P$ on a Finite Interval}

In this section we are concerned with the construction of the solution of $A C P$. Let $A \in \mathfrak{S}(X), k \in \mathbb{Z}_{+}, \omega \in \mathbb{R}$ and $T>0$, and let us consider the following conditions: 
$(I ; \omega) \quad\{\xi ; \xi \in \mathbf{R}, \xi>\omega\} \subset \rho(A)$,

$\left(I I_{T} ; k\right)$ there exists a number $M$ such that

$$
\left\|\xi^{n} R(\xi ; A)^{n}\right\|_{k} \leqq M \quad \text { for } \xi>\omega \text { and } n / \xi \in[0, T] \text {. }
$$

We then denote by $\mathcal{B S}_{1}(\omega, k, T)$ the family of all closed operators in $X$ satisfying conditions $(I ; \omega)$ and $\left(I I_{T} ; k\right)$. The purpose of this section is to discuss the construction of the solution of $A C P$ formulated for an operator belonging to this class $\mathscr{B}_{1}(\omega, k, T)$.

Proposition 2.1. Let $A \in \subseteq(X), k \in \mathbf{Z}_{+}, \omega \in \mathbf{R}$ and $T>0$, then the following conditions are equivalent:

(i) $A \in \mathscr{B}_{1}(\omega, k, T)$,

(ii) a) for each $\xi>\omega, \xi-A$ is an algebraic isomorphism of $D(A)$ onto $X$;

b) for $x \in D\left(A^{k}\right), \quad\left\{\xi^{n}(\xi-A)^{-n} x ; \xi>\omega, n / \xi \in[0, T]\right\} \quad$ is bounded in $X$,

(iii) $\rho(A) \neq \varnothing, \quad N(\xi-A)=\{0\}, \quad R(\xi-A) \supset D\left(A^{k}\right)$ for $\xi>\omega$ and condition (ii-b) holds.

Proof. (i) $\Rightarrow$ (ii) and (i) $\Rightarrow$ (iii) are evident.

(ii) $\Rightarrow(\mathrm{i})$ : For $\xi>\omega,(\xi-A)^{-1}$ is defined as a closed operator defined on $X$, and so $(\xi-A)^{-1} \in \mathfrak{B}(X)$ by the closed graph theorem. This means that $(I ; \omega)$ holds. Since $\rho(A) \neq \varnothing$, it follows (Dunford-Schwartz $[5 ;$ Th. VII 9.7]) that $A^{n} \in \mathfrak{S}(X)$ for $n \in \mathbb{Z}_{+}$. Hence, for each $n \in \mathbf{Z}_{+}, D\left(A^{n}\right)$ is a Banach space with respect to the norm \|\|$_{n}$. Thus, for any $n \in \mathbb{Z}_{+}$and $l \geqq k, R(\xi ; A)^{n} \in \mathfrak{B}\left(D\left(A^{l}\right), X\right)$. Hence, (ii-b) and the resonance theorem imply that $M=\sup \left\{\left\|\xi^{n} R(\xi ; A)^{n}\right\|_{k} ; \xi>\omega, n / \xi \in[0, T]\right\}<+\infty$.

(iii) $\Rightarrow$ (i): Let $\lambda_{0} \in \rho(A)$ and $x \in X$, then $R\left(\lambda_{0} ; A\right)^{k} x \in D\left(A^{k}\right)$. Let $\xi>\omega$ and $z=(\xi-A)^{-1} R\left(\lambda_{0} ; A\right)^{k} x$, then $z \in D\left(A^{k+1}\right)$ and $x=\left(\lambda_{0}-A\right)^{k}(\xi$ $-A) z=(\xi-A)\left(\lambda_{0}-A\right)^{k} z \in R(\xi-A)$. This means that $R(\xi-A)=X$. Since $A$ is closed, the closed graph theorem yields that $\xi \in \rho(A)$ provided $\xi>\omega$. Thus, $(I ; \omega)$ is satisfied. Hence, by the same argument as in the proof of (ii) $\Rightarrow(\mathrm{i})$, we have that $A \in \mathbb{S}_{1}(\omega, k, T)$.

Q.E.D.

Lemma 2.2. Let $A \in \mathscr{S}_{1}(\omega, k . T)$, then $D\left(A^{n}\right)$ is dense in $D\left(A^{2 k+1}\right)$ with respect to the norm \|\|$_{k}$ for $n \geqq 2 k+1$. 
Proof. Let $p \geqq 2 k+1$ and $x \in D\left(A^{p}\right)$, then $J_{h} x=(I-h A)^{-1} x \in D\left(A^{p \perp 1}\right)$ for $h$ sufficiently small and $J_{h} x-x=h J_{h} A x$. Thus, $\left\|J_{h} x-x\right\| \leqq h M\|A x\|_{k}$ and $\left\|A^{l} J_{h} x-A^{l} x\right\| \leqq h M\left\|A^{l+1} x\right\|_{k}$ for $l=1,2, \ldots, k$. This means that $D\left(A^{p+1}\right)$ is dense in $D\left(A^{p}\right)$ with respect to the norm \|\|$_{k}$ for $p \geqq 2 k+1$. Therefore, by induction, we have the assertion.

Q.E.D.

Our main theorem of this section is the following:

Theorem 2.3. Let $A \in \mathbb{S}_{1}(\omega, k, T)$ for some $\omega \in \mathbf{R}, k \in \mathbb{Z}_{+}$and $T>0$. Let $m=2 k+1$. Then there is a one-parameter family $\left\{U_{t} ; t \in[0, T]\right\} \subset \mathfrak{B}$ $\left(D\left(A^{m}\right), X\right)$ such that

(a) for every $x \in D\left(A^{m}\right)$ and $t \in[0, T]$,

$$
U_{t} x=\lim _{h \rightarrow+0}(I-h A)^{-[t / h]} x
$$

and the convergence is uniform with respect to $t \in[0, T]$;

(b) $\quad\left\|U_{t} x\right\| \leqq M\|x\|_{k} \quad$ for $t \in[0, T]$ and $x \in D\left(A^{m}\right)$,

(c) for every integer $p \geqq 1$, each $U_{t}$ maps $D\left(A^{m+p}\right)$ into $D\left(A^{p}\right)$ and

$$
A^{p} U_{t} x=U_{t} A^{p} x \quad \text { for } t \in[0, T] \text { and } x \in D\left(A^{m+p}\right) \text {; }
$$

(d) for $x \in D\left(A^{2 m}\right)$ and $t, s \in[0, T]$ with $t+s \in[0, T], U_{t+s} x$ $=U_{t} U_{s} x$

(e) for every $p \in \mathbb{Z}_{+}$and $x \in D\left(A^{m+1+p}\right)$, there is a number $\beta_{x, p}>0$ such that $\left\|_{\mid} U_{t} x-U_{s} x\right\|_{p} \leqq \beta_{x, p}|t-s|$ for $t, s \in[0, T]$, and furthermore, for every $x \in D\left(A^{m+1}\right)$ and $t \in[0, T]$,

$$
U_{t} x-x=\int_{0}^{t} U_{s} A x d s=\int_{0}^{t} A U_{s} x d s .
$$

Proof. (a) Let $x \in D\left(A^{2 k+2}\right)$ and $t \in[0, T]$. Let $\sigma^{-1} \geqq h^{-1}>$ $\max \{0, \omega\}$, and let us denote $J_{h}=(I-h A)^{-1}, h^{-1}>\max \{0, \omega\}$. Then we can write

$$
\left\|J_{\sigma}^{[t / \sigma]} x-J_{h}^{[t / h]} x\right\| \leqq\left\|J_{\sigma}^{[t / \sigma]} x-J_{\sigma}^{n_{\nu}} x\right\|+\left\|J_{\sigma}^{n_{\nu}} x-J_{h}^{\nu} x\right\|+\left\|J_{h}^{\nu} x-J_{h}^{[t / h]} x\right\| .
$$

Now, assume that $n \sigma \leqq h$ and $h \nu \in[0, T]$, then we have 


$$
\begin{aligned}
& J_{\sigma}^{n \nu} x-J_{h}^{\nu} x=-\sum_{i=0}^{\nu-1}\left\{J_{h}^{\nu-i} J_{\sigma}^{n i} x-J_{h}^{\nu-i-1} J_{\sigma}^{n(i+1)} x\right\}, \text { and } \\
& J_{h}^{\nu-i} J_{\sigma}^{n i} x-J_{h}^{\nu-i-1} J_{\sigma}^{n(i+1)} x=h J_{h}^{\nu-i} J_{\sigma}^{n(i+1)} A x-J_{h}^{\nu-i}\left\{J_{\sigma}^{n(i+1)} x-J_{\sigma}^{n i} x\right\} \\
= & (h-n \sigma) J_{h}^{\nu-i} J_{\sigma}^{n(i+1)} A x+J_{h}^{\nu-i}\left\{n \sigma J_{\sigma}^{n(i+1)} A x-\sigma \sum_{p=1}^{n} J_{\sigma}^{n i+p} A x\right\} \\
= & (h-n \sigma) J_{h}^{\nu-i} J_{\sigma}^{n(i+1)} A x+J_{h}^{\nu-i} \sigma^{2} \sum_{p=1}^{n-1} \sum_{q=p}^{n-1} J_{\sigma}^{n i+q+1} A^{2} x=I_{1}(\nu, i)+I_{2}(\nu, i) .
\end{aligned}
$$

Taking $\sigma=2^{-r}, h=2^{-r^{\prime}}, n=2^{r-r^{\prime}}$ and $\nu=\left[t 2^{r^{\prime}}\right]$, we note that $\mid[t / \sigma]-n$ $[t / h] \mid \leqq n$ and $h-n \sigma=0$. Also, using $\left(I I_{T} ; k\right)$ we see that

$$
\begin{aligned}
& N(A x) \equiv \sup \left\{\left\|J_{\sigma}^{j} A x\right\|_{k} ; \sigma>\omega, 0 \leqq j \sigma \leqq T\right\}<+\infty \\
& N\left(A^{2} x\right) \equiv \sup \left\{\left\|J_{\sigma}^{j} A^{2} x\right\|_{k} ; \sigma>\omega, 0 \leqq j \sigma \leqq T\right\}<+\infty
\end{aligned}
$$

Hence, we have that $\left\|I_{1}(\nu, i)\right\|=0$ and

$$
\left\|I_{2}(\nu, i)\right\| \leqq\left\|J_{h}^{\nu-i}\right\|_{k} \sigma^{2} \sum_{p=1}^{n-1} \sum_{q=p}^{n-1}\left\|J_{\sigma}^{n i+q+1} A^{2} x\right\|_{k} \leqq M h^{2} N\left(A^{2} x\right)
$$

and so, $\left\|J_{\sigma}^{n_{\nu}} x-J_{h}^{\nu} x\right\| \leqq M T N\left(A^{2} x\right) h$. Consequently, (2.1) is estimated as

$$
\left\|J_{\sigma}^{[t / \sigma]} x-J_{h}^{[t / h]} x\right\| \leqq n \sigma N(A x)+M T N\left(A^{2} x\right) h \leqq \operatorname{const}(x) \cdot h
$$

for $\sigma=2^{-r}$ and $h=2^{-r^{\prime}}$ with $r \geqq r^{\prime}$. This means that

$$
U_{t} x=\lim _{r \rightarrow \infty}\left(I-2^{-r} A\right)^{-\left[t 2^{r}\right]} x
$$

exists uniformly for $t \in[0, T]$. By using $\left(I I_{T} ; k\right)$ and Lemma 2.2, this convergence holds for every $x \in D\left(A^{2 k+1}\right)$. Now, we show that the limit is independent of the sequence chosen. Let $x \in D\left(A^{2 k+1}\right)$. Take any $\varepsilon>0$ and let $0 \leqq t \leqq T-\varepsilon$ and $0<\sigma \leqq h<\varepsilon$. Taking, this time, $\nu=[t / h]+1$ and $n=[[t / \sigma] / \nu]$, we observe that

$$
\left\{\begin{array}{l}
h \nu \leqq t+h, n \sigma \leqq h,|t-n \sigma \nu| \leqq 2 \sigma+T \sigma / h \\
|[t / \sigma]-n \nu| \sigma \leqq \sigma+T \sigma / h, \nu|n \sigma-h| \leqq h+2 \sigma+T \sigma / h
\end{array}\right.
$$

In fact, $h \nu=([t / h]+1) h \leqq t+h, n \sigma \leqq[t / \sigma] \sigma / \nu \leqq[t / \sigma] \sigma h / t \leqq h, n \nu=[[t /$ $\sigma] / \nu] \nu \leqq[t / \sigma], \quad 0 \leqq[t / \sigma]-n \nu \leqq[t / \sigma]-([t / \sigma] / \nu-1) \nu=[t / h]+1$, and 
$0 \leqq \nu h-\nu n \sigma=\nu h-t+t-n \nu \sigma \leqq h+2 \sigma+T \sigma / h$. Similarly, as above, taking $\sigma=2^{-r}$, then letting $r \rightarrow \infty$, we see using (2.2), (2.3) and (2.4) that

$$
\begin{aligned}
\| U_{t} x- & J_{h}^{[t / h]} x\left\|\leqq \varlimsup_{r \rightarrow \infty}\right\| J_{2}^{\left[\frac{t 2}{r}{ }^{r}\right]} x-J_{2^{-r} x}^{n \nu} \| \\
& \quad+\varlimsup_{r \rightarrow \infty}\left\|J_{2^{-r}}^{n \nu} x-J_{h}^{\nu} x\right\|+\left\|J_{h}^{\nu} x-J_{h}^{[t / h]} x\right\| \leqq \operatorname{const}(x) \cdot h,
\end{aligned}
$$

note that in this case $\left\|I_{1}(\nu, i)\right\| \leqq \nu|h-n \sigma| M N(A x)$. Therefore, we see that $\lim _{h \rightarrow+0} \sup _{t \in\left[0, T^{-} \varepsilon\right]}\left\|U_{t} x-J_{h}^{[t / h]} x\right\|=0$, and hence we have the assertion (a).

(b) follows from $\left(I I_{T} ; k\right)$ and (2.3).

(c) For $t \in[0, T], p \in \mathbb{Z}_{+}$and $x \in D\left(A^{m+p}\right)$, we have that $A^{p} J_{h}^{[t / h]} x$ $=J_{h^{[t / h]}} A^{p} x$ for $h^{-1}>\max \{0, \omega\}$. Assume $p=1$, then $A x \in D\left(A^{m}\right)$. Since $A \in \mathfrak{S}(X)$, (a) implies that $U_{t} x \in D(A)$ and $U_{t} A x=A U_{t} x$. Assume $p=2$,

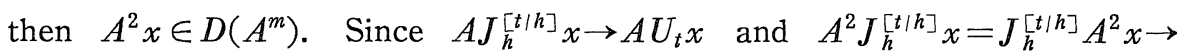
$U_{t} A^{2} x$, again the closedness of $A$ implies that $A^{2} U_{t} x=U_{t} A^{2} x$. Inductively, we can prove (c) for all $p \in \mathbb{Z}_{+}$.

(d) Let $x \in D\left(A^{2 m}\right)$ and $s, t, s+t \in[0, T]$. Then $\left(I I_{T} ; k\right)$ yields that $\left\|J_{h}^{[(s+t) / h]} x-J_{h}^{[s / h]+[t / h]} x\right\| \leqq M\|A x\|_{k} \cdot h$. Note that $U_{t} x \in D\left(A^{m}\right)$ for all $t \in[0, T]$ by (c) and that $\lim _{h \rightarrow+0}\left\|J_{h}^{[t / h]} x-U_{t} x\right\|_{k}=0$ by (a) and (c). Hence, (d) is proved by estimating

$$
\begin{aligned}
\left\|U_{s+t} x-U_{s} U_{t} x\right\|_{\|} \leqq \| U_{s+t} x & -J_{\bar{h}}^{[(s+t) \mid h]} x\|+M\| A x \|_{k} \cdot h \\
& +M\left\|J_{\bar{h}}^{[t / h]} x-U_{t} x\right\|_{k}+\left\|J_{h}^{[s / h]} U_{t} x-U_{s} U_{t} x\right\| .
\end{aligned}
$$

(e) Let $x \in D\left(A^{m+1}\right)$, then condition $\left(I I_{T} ; k\right)$ states that $J_{\bar{h}}^{[s / h]} A x$ are step functions on $[0, T]$ and are uniformly bounded with respect to $s \in$ $[0, T]$ and $h>0$ sufficiently small. Since

$$
\begin{gathered}
h \sum_{k=0}^{[t / h]-1} J_{h}^{h} A x=J_{h}^{\lfloor t / h]} x-x+h A x-h J_{h}^{[t / h]} A x, \\
J_{h}^{[t / h]} x-x=\int_{0}^{t} J_{h}^{[s / h]} A x d s-\int_{[t / h] h}^{t} J_{h}^{[s / h]} A x d s-h A x+h J_{h}^{[t / h]} A x .
\end{gathered}
$$

In view of this, (a), (c) and the dominated convergence theorem imply that

$$
U_{t} x-x=\int_{0}^{t} U_{s} A x d s=\int_{0}^{t} A U_{s} x d s
$$


Now, let $p \in \mathbf{Z}_{+}$and $x \in D\left(A^{m+p+1}\right)$. Since $A^{p} x \in D\left(A^{m+1}\right)$ and $U_{t} A^{p} x=$ $A^{p} U_{t} x$ for $t \geqq 0$, we see setting $\beta_{x, p}=\sup _{0 \leqq s \leqq T}\left\|U_{s} A x\right\|_{p}(<+\infty)$ that $\| U_{t} x$ $-U_{s} x \|_{p} \leqq \beta_{x, p}|t-s|$ for $t, s \in[0, T]$.

Q.E.D.

Lemma 2.4. Let $A \in \mathbb{S}(X)$. Assume that $\rho(A) \neq 0$ and that for some $m \in \mathbb{Z}_{+}$, there is a one-parameter family $\left\{U_{t} ; t \in[0, T]\right\} \subset \mathfrak{B}\left(D\left(A^{m}\right)\right.$, $X)$ with the properties (a)-(e) stated in Theorem 2.3. Then for $x \in D$ $\left(A^{m+1}\right), U_{t} x$ becomes a unique solution of $A C P_{1}$ formulated for $A$ on $[0$, $T]$ satisfying the initial condition $U_{0} x=x$.

Proof. In view of the property (e) stated in Theorem 2.3, for each $x \in D\left(A^{m+1}\right), u(t ; x)=U_{t} x$ becomes a solution of $A C P_{1}$ for $A$ associated with the initial value $x$. To prove the uniqueness of the solution, we employ Phillips' method: Let $t, t-s \in[0, T]$, then it follows from Theorem $2.3(\mathrm{c})$ and (e) that

$$
U_{t} A x=A U_{t} x \text { and }(\partial / \partial s) U_{t-s} x=-A U_{t-s} x \quad \text { for } x \in D\left(A^{m+1}\right) \text {. }
$$

Let $u(t)$ be another solution of $A C P_{1}$ for $A$ associated with the same initial value $x$ and then put $v(t)=U_{t} x-u(t), t \in[0, T]$; note that $v(0)$ $=0$. Now, let $\lambda_{0} \in \rho(A)$, then $R\left(\lambda_{0} ; A\right)^{m} v(s) \in D\left(A^{2 k+2}\right)$ and $(\partial / \partial s) U_{t-s} R$ $\left(\lambda_{0} ; A\right)^{m} v(s)=-A U_{t-s} R\left(\lambda_{0} ; A\right)^{m} v(s)+U_{t-s} R\left(\lambda_{0} ; A\right)^{m} A v(s)=-A U_{t-s} R$ $\left(\lambda_{0} ; A\right)^{m} v(s)+A U_{t-s} R\left(\lambda_{0} ; A\right)^{m} v(s)=0$ for $t, t-s \in[0, T]$. Therefore,

$$
0=\int_{0}^{t}(\partial / \partial s) U_{t-s} R\left(\lambda_{0} ; A\right)^{m} v(s) d s=U_{0} R\left(\lambda_{0} ; A\right)^{m} v(t)-U_{t} R\left(\lambda_{0} ; A\right)^{m} v(0)=R
$$

$\left(\lambda_{0} ; A\right)^{m} v(t)$ for $t \in[0, T]$. This means that $v(t) \equiv 0$.

Remark 2.5. The proof mentioned above shows that the family $\left\{U_{t}\right.$; $t \in[0, T]\}$ satisfying (a)-(e) of Theorem 2.3 is uniquely determined.

By virtue of Lemma 2.4, we obtain the following result on the existence of the solution of $A C P$.

Theorem 2.6. Let $A \in \mathbb{S}_{1}(\omega, k, T)$ for some $\omega \in \mathbf{R}, k \in \mathbf{Z}_{+}$and $T>0$. Then for $x \in D\left(A^{2 k+2}\right)$ there is a unique solution $u(t ; x)$ of $A C P_{1}$ formulated for $A$ on $[0, T]$ satisfying $u(0 ; x)=x$.

Next, we consider the case in which $A$ is densely defined.

Lemma 2.7. Let $A$ be a densely defined, closed operator in $X$ with non-empty resolvent set. Then for every pair of integers $m$ and $k$ with 
$m \geqq k \geqq 0, D\left(A^{m}\right)$ is dense in $\left[D\left(A^{k}\right)\right]$; that is, $D\left(A^{m}\right)$ is a core of $A^{k}$ for $m \geq k \geqq 0$.

Proof. Fix a $k \in \mathbf{Z}_{+}$. Since the lemma is evident in case of $m=k$, we assume that $m>k$. Let $m>n \geqq k$. We first show that $D\left(A^{n+1}\right)$ is dense in $D\left(A_{n}\right)$ with respect to the norm $\left.\right|_{1} \|_{1}$. Let $x \in D\left(A^{n}\right)$ and $\mu \in \rho$ $(A)$, then we can find a $y \in X$ such that $x=R(\mu ; A)^{n} y$. Since $\left.\overline{D(A}\right)=X$, there is a sequence $\left\{y_{p}\right\} \subset D(A)$ with $y_{p} \rightarrow y$. Hence, it follows that $R(\mu$; $A)^{n} y_{p} \in D\left(A^{n+1}\right), R(\mu ; A)^{n} y_{p} \rightarrow R(\mu ; A)^{n} y=x$, and also that $A^{l} R(\mu ; A)^{n}$ $y_{p}=[A R(\mu ; A)]^{l} R(\mu ; A)^{n-l} y_{p} \rightarrow[A R(\mu ; A)]^{l} R(\mu ; A)^{n-l} y=A^{l} x$ for $1 \leqq l$ $\leqq k$. This means that $D\left(A^{n+1}\right)$ is dense in $D\left(A^{n}\right)$ in the sense of \|\|$_{k^{-}}$ norm. Now, let $x \in D\left(A^{k}\right)$ and $\varepsilon>0$, then putting $n=k$ in the above argument we can find an $x_{k+1} \in D\left(A^{k+1}\right)$ such that $\left\|x-x_{k+1}\right\|_{k}<\varepsilon /(m-k)$. Next, letting $n=k+1$ we can find an $x_{k+2} \in D\left(A^{k+2}\right)$ with $\left\|x_{k+1}-x_{k+2}\right\|_{k}$ $<\varepsilon /(m-k)$. Inductively, a set of points $\left\{x_{n} ; k<n \leqq m\right\}$ can be found such that $x_{n} \in D\left(A^{n}\right)$ and $\left\|x_{n}-x_{n+1}\right\|_{k}<\varepsilon /(m-k)$ for $k<n<m$. Hence, $\left\|x-x_{m}\right\|_{k}<\varepsilon$. This means that $D\left(A^{m}\right)$ is dense in $D\left(A^{k}\right)$ in the sense of $\|\cdot\|_{k}$.

Q.E.D.

Theorem 2.8. Let $A \in \mathbb{S}_{1}(\omega, k, T)$ for some $\omega \in \mathbb{R}, k \in \mathbb{Z}_{+}$and $T>0$. If $A$ is densely defined, then there is a one-parameter family $\left\{U_{t}\right.$; $t \in[0, T]\} \subset \mathfrak{B}\left(D\left(A^{k}\right), X\right)$ such that (a)-(e) of Theorem 2.3 hold for $m=k$.

Proof. Since $D\left(A^{2 k+1}\right)$ is dense in $\left[D\left(A^{k}\right)\right]$ by Lemma 2.7 , condition $\left(I I_{T} ; k\right)$ implies that the convergence (2.3) holds for all $x \in D\left(A^{k}\right)$, uniformly for $t \in[0, T]$. Hence, it follows from $\left(I I_{T} ; k\right)$ that for every $x \in D\left(A^{k}\right)$ and $t \in[0, T], U_{t} x=\lim (I-h A)^{-[t / h]} x$ exists, and so $\left\|U_{t} x\right\|$ $\leqq M\|x\|_{k}$ for $x \in D\left(A^{k}\right)$ and $t \in[0, T]$. This means that $U_{t} \in \mathfrak{B}\left(D\left(A^{k}\right), X\right)$ for $t \in[0, T]$. Therefore, we may replace $m=2 k+1$ in the proofs of (c)-(e) of Theorem 2.3.

Q.E.D.

Corollary 2.9. If $A$ is a densely defined operator belonging to $\mathscr{S}_{1}$ $(\omega, k, T)$ for some $\omega \in \mathbb{R}, k \in \mathbb{Z}_{+}$and $T>0$, then for every $x \in D\left(A^{k+1}\right)$ there is a unique solution $u(t ; x)$ of $A C P_{1}$ formulated for $A$ on $[0, T]$ such that $u(0 ; x)=x$.

The proof follows from Theorem 2.8 and Lemma 2.4 . 
Corollary 2.10. Let $A$ be a densely defined, closed operator in $X$ for which there exist an $\omega \in \mathbb{R}$ and $k \in \mathbb{Z}_{+}$such that $N(\xi-A)=\{0\}$ and $R(\xi-A) \supset D\left(A^{k}\right)$ for $\xi>\omega$. If $\rho(A) \neq \varnothing$ and the set $\left\{\xi^{n} R(\xi ; A)^{n} x ; \xi>\omega\right.$, $n / \xi \in[0, T]\}$ is bounded for every $x \in D\left(A^{k}\right)$, then for every $x \in D\left(A^{k+1}\right)$, there exists a unique solution $u(t ; x)$ of $A C P_{1}$ for $A$ on $[0, T]$ satisfying the initial condition $u(0 ; x)=x$.

The proof follows from Proposition 2.1 and Corollary 2.9.

\section{Construction of the Solution in a Frécht Space}

In the preceding section we treated classes $\mathcal{S}_{1}(\omega, k, T), k \in \mathbb{Z}_{+}$. In this section we introduce another class $\mathcal{S}_{1}(\omega, \infty, T)$ of closed operators and discuss the construction of the solution of $A C P$ on a finite interval.

For given $\omega \in \mathbb{R}$ and $T>0$, we denote by $\mathscr{E S}_{1}(\omega, \infty, T)$ the class of all closed operators $A$ satisfying conditions $(I ; \omega)$ and

$\left(I I_{T} ; \infty\right)$ Let $Y=Y_{A}=\bigcap_{n \geqq 1} D\left(A^{n}\right)$; then there exist a $k=k_{A} \in \mathbb{Z}_{+}$and an $M=M_{A}>0$ such that

$$
\left\|\xi^{n} R(\xi ; A)^{n} y\right\| \leqq M\|y\|_{k} \quad \text { for } y \in Y, \xi>\omega \text { and } n / \xi \in[0, T] \text {. }
$$

Note that $\mathbb{S}_{1}(\omega, \infty, T) \supset \mathbb{S}_{1}(\omega, k, T)$ for every $k \in \mathbb{Z}_{+}$. Now, let $A \in$ $\mathcal{S}_{1}(\omega, \infty, T)$ and we introduce to $Y\left(=Y_{A}\right)$ a locally convex topology defined in the following lemma; we denote the space by the same symbol $Y$.

Lemma 3.1. Let $A \in \mathscr{S}_{1}(\omega, \infty, T)$. Then we have:

(a) $Y=\bigcap_{n \geqq 1} D\left(A^{n}\right)$ is a Fréchet space with respect to the seminorm system $\left\{p_{n}(x) \stackrel{n \geqq 1}{=}\left\|A^{n} x\right\| ; n \in \mathbb{Z}_{+}\right\}$;

(b) $A \mid Y \in \mathbb{R}(Y)$ and $R(\xi ; A) \mid Y=(\xi-A \mid Y)^{-1} \in \mathbb{R}(Y)$ for $\xi>\omega$;

(c) $\xi^{n} R(\xi ; A)^{n} \mid Y \in \mathbb{R}(Y)$ for $\xi>\omega$ and $n \in \mathbb{Z}_{+}$and the family $\left\{\xi^{n} R\right.$ $\left.(\xi ; A)^{n} \mid Y ; \xi>\omega, n / \xi \in[0, T]\right\}$ is equicontinuous on $Y$.

Proof. (a) $Y$ is a locally convex space with respect to the countable system of seminorms, $p_{n}, n \in \mathbb{Z}_{+}$, and hence $Y$ is an invariant metric space. Also, it is easily seen from the closedness of $A$ that $Y$ is complete with respect to the metric. 
(b) The first part is evident from the definition of the topology. To prove the second part, we note that $\xi-A$ maps $Y$ onto itself in a oneto-one manner for each $\xi>\omega$.

(c) The first part is clear from (b). For every $p_{m}$, condition $\left(I I_{T} ; \infty\right)$ states that $p_{m}\left(\xi^{n} R(\xi ; A)^{n} y\right)=\left\|\xi^{n} R(\xi ; A)^{n} A^{m} y\right\| \leqq M\left\|A^{m} y\right\|_{k} \leqq$ $M\|y\|_{k+m}$. This implies that for every continuous seminorm $p$ on $Y$ there exists a continuous seminorm $q$ on $Y$ such that $p\left(\xi^{n} R(\xi ; A)^{n} y\right) \leqq q(y)$ for $y \in Y, \xi>\omega, n \in \mathbb{Z}_{+}$and $n / \xi \in[0, T]$.

Remark 3.2. (a) In view of Lemma 3.1(b), we can regard $R(\xi ; A)$ as an element of $\mathfrak{L}(Y)$ when we treat it in the space $Y$.

(b) Condition $\left(I I_{T} ; \infty\right)$ is equivalent to $\left(I I_{T} ; \infty\right)^{\prime}$ for every $y \in Y, \sup \left\{\left\|\xi^{n} R(\xi ; A)^{n} y\right\| ; \xi>\omega, n / \xi \in[0, T]\right\}<+\infty$. In fact, if $\left(I I_{T} ; \infty\right)^{\prime}$ holds, then, in view of Lemma 3.1(a), we see applying the principle of uniform boundedness that (c) of Lemma 3.1 holds. But, by virtue of the definition of the topology of $Y$, this means that condition $\left(I I_{T} ; \infty\right)$ is satisfied.

Theorem 3.3. Let $A \in \mathbb{S}_{1}(\omega, \infty, T)$, then there exist $a k \in \mathbb{Z}_{+}$and a one-parameter family $\left\{U_{t} ; t \in[0, T]\right\} \subset \mathbb{S}(Y)$ such that

(a) for every $y \in Y, U_{t} y=\lim _{h \rightarrow+0}(I-h A)^{-[t / h]} y$ holds uniformly for $t \in[0, T]$ with respect to the topology of $Y$;

(b) the family $\left\{U_{t} ; t \in[0, T]\right\}$ is equicontinuous on $Y$;

(c) for every $l \in \mathbb{Z}_{+}, t \in[0, T]$ and $y \in Y, A^{l} U_{t} y=U_{t} A^{l} y$;

(d) for every $y \in Y$ and $t, s \in[0, T]$ with $t+s \in[0, T], U_{t+s} y$ $=U_{t} U_{s} y$;

(e) for every $p_{m}, p_{m}\left(U_{t} y-U_{s} y\right) \leqq|t-s| M\|y\|_{k+m+1}$ for $y \in Y$ and $t, s \in[0, T]$; and furthermore, for every $y \in Y$ and $t \in[0, T]$,

$$
U_{t} y-y=\int_{0}^{t} U_{s} A y d s=\int_{0}^{t} A U_{s} y d s
$$

Proof. First, by the same way as in the proof of Theorem 2.3(a), we obtain

$$
U_{t} y=\lim _{h \rightarrow+0}(I-h A)^{-[t / h]} y
$$


where the convergence holds uniformly for $t \in[0, T]$. Thus we can define a one-parameter family $\left\{U_{t} ; t \in[0, T]\right\}$ of operators from $Y$ into $X$. We demonstrate that this family has the properties (a)-(e) mentioned above. Since $A^{n} \in \mathcal{C}(X)$ and $A^{n}(Y) \subset Y$ for $n \in \mathbf{Z}_{+}$, it follows that $U_{t} y$ $\in D\left(A^{n}\right)$ and $U_{t} A^{n} y=A^{n} U_{t} y$ for $y \in Y, n \in \mathbb{Z}_{+}$and $t \in[0, T]$. Hence, $U_{t}$ maps $Y$ into itself and (c) holds. Therefore, we see using (3.1) that for every $p_{m}$ and $y \in Y, p_{m}\left(U_{t} y-J_{h}^{[t / h]} y\right)=\left\|U_{t} A^{m} y-J_{h}^{[t / h]} A^{m} y\right\| \rightarrow 0$ as $h \rightarrow+0$, uniformly for $t \in[0, T]$. This means that (a) holds. Also, condition $\left(I I_{T} ; \infty\right)$ states that $\left\|U_{t} y\right\| \leqq M \|_{k}$ for $y \in Y$ and $t \in[0, T]$. Hence, for every $p_{m}$ and $y \in Y, p_{m}\left(U_{t} y\right)=\left\|U_{t} A^{m} y\right\| \leqq M\|y\|_{k+m}$, which yields the property (b). (d) follows from (3.1) and (b), in a similar way to the proof of Theorem 2.3 (d). Finally, we show (e). Let $0 \leqq s<t \leqq T$, $0<h<t-s$, and $y \in Y$. Then, as mentioned in the proof of Theorem 2.3 (e), $J_{\bar{h}}^{[t / h]} y-J_{\bar{h}}^{[s / h]} y=\Sigma_{i=[s / h]+1}^{[t / h]} J_{h}^{i} A y$ and $h|[t / h]-[s / h]| \leqq t-s+h$; and hence for every $p_{m}$ and $y \in Y$,

$$
p_{m}\left(J_{\bar{h}}^{[t / h]} y-J_{\bar{h}}^{[s / h]} y\right) \leqq(t-s+h) M\left\|A^{m+1} y\right\|_{k} .
$$

Passing to the limit as $h \rightarrow+0$, we obtain the first assertion of (e). Hence, for every $y \in Y, U_{t} y$ is continuous on $[0, T]$ with respect to the topology of $Y$. Therefore, $U_{t} y$ is integrable over any interval $[0, t] C$ $[0, T]$ in the sense of Riemann. On the other hand, relation (2.6) and the Lebesgue convergence theorem imply that for every $y^{*} \in Y^{*}$,

$$
\begin{aligned}
& <U_{t} y-y, y^{*}>=\int_{0}^{t}<U_{s} A y, y^{*}>d s=<\int_{0}^{t} U_{s} A y d s y^{*}> \\
& =<\int_{0}^{t} A U_{s} y d s, y^{*}>.
\end{aligned}
$$

Hence, we have the last assertion of (e).

Q.E.D.

Remark 3.4. In view of Theorem 3.3 (e) and Lemma 3.1, we see that $U_{t} y$ is infinitely differentiable (with respect to both topologies of $X$ and $Y$ ) and $(d / d t)^{n} U_{t} y=A^{n} U_{t} y=U_{t} A^{n} y$ for $t \in[0, T]$.

Corollary 3.5. Let $A \in \mathbb{S}_{1}(\omega, \infty, T)$ for some $\omega \in \mathbf{R}, k \in \mathbb{Z}_{+}$and $T>0$. Then for every $y \in Y$, there is a unique solution $u(t ; y)$ of $A C P_{1}$ on $[0, T]$ for $A \mid Y$ in the Fréchet space $Y$ such that $u(0 ; y)=y$. Fur- 
thermore, this $u(t ; y)$ is also a unique solution of $A C P_{1}$ on $[0, T]$ for $A$ in $X$ with the initial value $y$.

Proof. By virtue of Theorem 3.3, $u(t ; y)=U_{t} y$ is the solution of $A C P_{1}$ formulated for $A \mid Y$ in $Y$. Since the topology of $Y$ is stronger than that of $X, u(t ; y)$ is also the solution of $A C P_{1}$ for $A$ in $X$. The unicity of the solution of each $A C P_{1}$ can be proved in a quite similar way to the proof of Lemma 2.4 .

Q.E.D.

Next, we consider the case in which $\bar{Y}=X$.

Lemma 3.6. Let $A$ be a closed operator with non-empty resolvent set. If $\bar{Y}=X$, then $Y$ is dense in $\left[D\left(A^{n}\right)\right]$ for each $n \in \mathbf{Z}_{+}$. Therefore, $Y$ is a core of $A^{n}, n \in \mathbf{Z}_{+}$.

Proof. For every $n \in \mathbf{Z}_{+}, x \in D\left(A^{n}\right)$ and $\lambda_{0} \in \rho(A)$, we can find a $y \in X$ with $x=R\left(\lambda_{0} ; A\right)^{n} y$. Since $\bar{Y}=X$, there is a sequence $\left\{y_{p}\right\} \subset Y$ such that $y_{p} \rightarrow y$ in $X$. Hence, $R\left(\lambda_{0} ; A\right)^{n} y_{p} \in Y$ and $R\left(\lambda_{0} ; A\right)^{n} y_{p} \rightarrow$ $R\left(\lambda_{0} ; A\right)^{n} y=x$. Also, $A^{l} R\left(\lambda_{0} ; A\right)^{n} y_{p}=\left[A R\left(\lambda_{0} ; A\right)\right]^{l} R\left(\lambda_{0} ; A\right)^{n-l} y_{p} \rightarrow[A R$ $\left.\left(\lambda_{0} ; A\right)\right]^{l} R\left(\lambda_{0} ; A\right)^{n-l} y=A^{l} x$ for $1 \leqq l \leqq n$. This means that $\| R\left(\lambda_{0} ; A\right)^{n} y_{p}$ $-x \|_{n} \rightarrow 0$. Therefore, $Y$ is dense in $\left[D\left(A^{n}\right)\right]$.

Q.E.D.

Theorem 3.7. Let $A \in \mathbb{S}_{1}(\omega, \infty, T)$ and $\left\{U_{t} ; t \in[0, T]\right\}$ be the corresponding one-parameter family obtained by Theorem 3.3. Assume that $\vec{Y}=X$ and that there exist a $k \in \mathbf{Z}_{+}$and $M>0$ such that $\left\|U_{t} y\right\| \leqq M\|y\|_{k}$ for $y \in Y$. Then $\left\{U_{t}\right\}$ can be extended to a one-parameter family $\left\{\widetilde{U}_{t}\right.$; $t \in[0, T]\} \subset \mathfrak{B}\left(D\left(A^{k}\right), X\right)$ such that (b)-(e) of Theorem 2.3 hold for $m=k$.

Proof. Let $x \in D\left(A^{k}\right)$. Since $Y$ is dense in $\left[D\left(A^{k}\right)\right]$ by Lemma 3.6, there is a sequence $\left\{y_{p}\right\} \subset Y$ such that $\left\|x-y_{p}\right\|_{k} \rightarrow 0$. Hence, $\left\|U_{t} y_{p}-U_{t} y_{p^{\prime}}\right\|$ $\leqq M\left\|y_{p}-y_{p}\right\|_{k} \rightarrow 0$. This means that $\widetilde{U}_{t} x=\lim _{p \rightarrow+\infty} U_{t} y_{p}$ exists uniformly for $t \in[0, T]$, note that the limit is independent of the sequence chosen. Hence, for each $t \in[0, T]$, we can define an operator $\tilde{U}_{t}$ on $D\left(A^{k}\right)$ in such a way that $\left\|\widetilde{U}_{t} x\right\| \leqq M\|x\|_{k}$ for $x \in D\left(A^{k}\right)$. We then show that (c)(e) of Theorem 2.3 hold for $m=k$. First, let $l \in \mathbf{Z}_{+}$and $x \in D\left(A^{k+l}\right)$, then we can find a sequence $\left\{y_{p}\right\} \subset Y$ such that $\left\|y_{p}-x\right\|_{k+l} \rightarrow 0$ as $p \rightarrow \infty$. Since $\left\|A^{l} y_{p}-A^{l} x\right\|_{k} \rightarrow 0$, we have that $\tilde{U}_{t} A^{l} x=\lim \tilde{U}_{t} A^{l} y_{p}=\lim U_{t} A^{l} y_{p}$ $=\lim A^{l} U_{t} y_{p}$. Since $U_{t} y_{p} \rightarrow \widetilde{U}_{t} x$, the closedness of ${ }^{p} A$ yields that $\tilde{U}_{t} A^{l} x=$ $A^{l} \stackrel{p}{\tilde{U}_{t}} x$. This implies (c). Next, let $x \in D\left(A^{2 k}\right)$, then there exists a sequence 
$\left\{y_{p}\right\} \subset Y$ such that $\left\|y_{p}-x\right\|_{2 k} \rightarrow 0$ as $p \rightarrow \infty$. Hence,

$$
\begin{aligned}
& \left\|\widetilde{U}_{t+s} x-\widetilde{U}_{t} \widetilde{U}_{s} x\right\| \leqq\left\|\widetilde{U}_{t+s} x-\widetilde{U}_{t+s} y_{p}\right\|+\left\|U_{t+s} y_{p}-U_{t} U_{s} y_{p}\right\| \\
& \quad+\left\|\widetilde{U}_{t} U_{s} y_{p}-\widetilde{U}_{t} \widetilde{U}_{s} x\right\| \leqq M\left\|x-y_{p}\right\|_{k}+M\left\|U_{s} y_{p}-\widetilde{U}_{s} x\right\|_{k} \\
& \leqq M(1+k M)\left\|x-y_{p}\right\|_{2 k} \quad \text { for all } p .
\end{aligned}
$$

This estimate yields (d). Finally, we prove (e). Let $x \in D\left(A^{k+1}\right)$. Then a sequence $\left\{y_{p}\right\} \subset Y$ can be found such that $\left\|x-y_{p}\right\|_{k+1} \rightarrow 0$. Hence, $\left\|A x-A y_{p}\right\|_{k} \rightarrow 0$, and so $A \widetilde{U}_{t} x=\widetilde{U}_{t} A x=\lim \tilde{U}_{t} A y_{p}=\lim A U_{t} y_{p}$ and the convergence holds uniformly for $t \in[0, T]^{p}$. The strong continuity of each $A U_{t} y_{p}$ with respect to $t$ implies that of $A \widetilde{U}_{t} x$. Since $U_{t} y_{p}-y_{p}=\int_{0}^{t} A U_{s} y_{p} d s$ for each $p$, passing to the limit as $p \rightarrow \infty$, we have that $\widetilde{U}_{t} x-x=$ $\int_{0}^{t} A \tilde{U}_{s} x d s$ for $t \in[0, T]$. This shows that (e) holds for $m=k$. Q.E.D.

Finally, we consider the relationship between $\mathscr{S}_{1}(\omega, \infty, T)$ and $\mathcal{S}_{1}(\omega, k, T)$.

Lemma 3.8. Let $A \in \mathfrak{C}(X), \overline{D(A)}=X$ and $Y=\bigcap_{n \geqq 1} D\left(A^{n}\right)$. Let $D_{T}$ be the set of all elements $x \in D(A)$ such that for each $x \in 1$ $X$-valued function $u(t ; x)$ satisfying

(i) $u(t ; x) \in D(A)$ for $t \in[0, T]$ and $A u(t ; x)$ is strongly continuous in $[0, T]$;

(ii) $u(t ; x)-x=\int_{0}^{t} A u(s ; x) d s$ for $t \in[0, T]$. Then, $\bar{D}_{T}=X$ implies $\bar{Y}=X$.

Proof. Let us denote by $D(0, T)$ the space of $\mathbf{R}$-valued $C^{\infty}$-functions whose supports are contained in $(0, T)$ and let $\mathfrak{D}_{T}=\left\{\int_{0}^{T} \phi(t) u(t ; x) d t\right.$; $\left.\phi \in D(0, T), x \in D_{T}\right\}$. We demonstrate that $\mathfrak{D}_{T} \subset Y$ and $\overline{\mathfrak{D}}_{T}=X$. Take $\phi \in$ $D(0, T)$ and $x \in D_{T}$ and then put $y=\int_{0}^{T} \phi(t) u(t ; x) d t$. Then $\phi^{(n)} \in D(0, T)$ for $n \in \mathbf{Z}_{+}$. Hence, we see using the closedness of $A$ that

$$
\begin{aligned}
& (-1)^{n} \int_{0}^{T} \phi^{(n)}(t) u(t ; x) d t=(-1)^{n-1} \int_{0}^{T} \phi^{(n-1)}(t) u^{\prime}(t ; x) d t \\
= & (-1)^{n-1} \int_{0}^{T} \phi^{(n-1)}(t) A u(t ; x) d t=A(-1)^{n-1} \int_{0}^{T} \phi^{(n-1)}(t) u(t ; x) d t,
\end{aligned}
$$

for $n \in \mathbf{Z}_{+} \backslash\{0\}$. This implies that $y \in D\left(A^{n}\right)$ and $A^{n} y=(-1)^{n} \int_{0}^{T} \phi^{(n)}(t) u$ 
$(t ; x) d t$ for $n \in \mathbf{Z}_{+}$. Hence, $\mathfrak{D}_{T} \subset Y$. Next, assume that $\overline{\mathfrak{D}}_{T} \varsubsetneqq X$, then there exists an $x_{0}^{*} \in X^{*} \backslash\{0\}$ such that $x_{0}^{*}\left[\mathfrak{D}_{T}\right]=\{0\}$. Thus, we obtain

$$
\int_{0}^{T} \phi(t)<u(t ; x), x_{0}^{*}>d t=<\int_{0}^{T} \phi(t) u(t ; x) d t, x_{0}^{*}>=0
$$

for $\phi \in D(0, T)$ and $x \in D_{T}$. But $\left\langle u(t ; x), x_{0}^{*}\right\rangle$ is continuous on $[0, T]$ and $\lim _{t \rightarrow+0} u(t ; x)=x$ for $x \in D_{T}$; and hence it follows that $\left\langle x, x_{0}^{*}\right\rangle=0$. Consequently, we obtain $x_{0}^{*}\left[D_{T}\right]=\{0\}$, contrary to the way in which $x_{0}^{*}$ was chosen.

Q.E.D.

Theorem 3.9. Let $A$ be a densely defined, closed operator belonging to $\mathbb{B}_{1}(\omega, \infty, T)$ and let $Y=\bigcap_{n \geqq 1} D\left(A^{n}\right)$. Then $A \in \mathcal{B}_{1}(\omega, k, T)$ for some $k \in \mathbf{Z}_{+}$if and only if $\bar{Y}=X$.

Proof. Assume that $\bar{Y}=X$. Then condition $\left(I I_{T} ; \infty\right)$ states that there exist a $k \in \mathbf{Z}_{+}$and an $M>0$ such that $\left\|\xi^{n} R(\xi ; A)^{n} y\right\| \leqq M\|y\|_{k}$ for $y \in Y, \xi>\omega$ and $n / \xi \in[0, T]$. On the other hand, $Y$ is dense in $\left[D\left(A^{k}\right)\right]$ by Lemma 3.6. Since $\xi^{n} R(\xi ; A)^{n} \in \mathfrak{B}(X)$ for $\xi>\omega$ and $n \in \mathbf{Z}_{+}$, we can extend the inequality for $y \in Y$ to that for $x \in D\left(A^{k}\right)$, in a similar way to the proof of Theorem 3.7. Therefore, $\left(I I_{T} ; k\right)$ is satisfied. Conversely, suppose that $A \in \mathcal{S}_{1}(\omega, k, T)$, then by Theorem 2.8 we can take $D_{T}=D$ $\left(A^{k+1}\right)$ in Lemma 3.8. Since $\overline{D\left(A^{k+1}\right)}=X$ by Lemma 2.7 , Lemma 3.8 yields that $\bar{Y}=X$.

Q.E.D.

Remark 3.10. By virtue of Theorem 3.9, we see that for every $A \in \mathcal{S}_{1}(\omega, \infty, T)$ such that $\bar{Y}=X$, the assumption of Theorem 3.7 is always satisfied for some $k \in \mathbf{Z}_{+}$. Hence, we have the following conclusion:

Theorem 3.7'. Let $A \in \mathbb{S}_{1}(\omega, \infty, T)$. If $\bar{Y}=X$, then there exist $a$ $k \in \mathbf{Z}_{+}$and a one-parameter family $\left\{U_{t} ; t \in[0, T]\right\} \subset \mathfrak{B}\left(D\left(A^{k}\right), X\right)$ such that (b)-(e) of Theorem 2.3 hold for $m=k$.

Remark 3.11. If $A \in \mathcal{S}_{1}(\omega, k, T)$ and if $\overline{D(A)}=X$, then $Y$ is a core of $A^{n}$ for each $n \in \mathbf{Z}_{+}$. This follows from Theorem 2.8, Lemma 3.8 and Lemma 3.6.

\section{Cauchy Problems on $[0, \infty)$}

In this section, we introduce some classes of closed operators and 
discuss the construction of the solution of $A C P$ formulated on $[0, \infty)$ for such an operator.

Let $A \in \mathbb{E}(X), \omega \in \mathbf{R}$ and $k \in \mathbf{Z}_{+}$. For this operator $A$ let us consider conditions $(I ; \omega)$ and

$(I I ; k)$ for every $T>0$, there is a number $M(T)$ such that

$$
\left\|\xi^{n} R(\xi ; A)^{n}\right\|_{k} \leqq M(T) \quad \text { for } \xi>\omega \text { and } 0 \leqq n / \xi \leqq T
$$

We denote by $\mathscr{S}_{1}(\omega, k)$ the collection of all closed operators in $X$ satisfying $(I ; \omega)$ and $(I I ; k)$ mentioned above. In view of proposition 2.1, condition $(I I ; k)$ is equivalent to the following condition:

$(I I ; k)^{\prime}$ for every $T>0$ and $x \in D\left(A^{k}\right),\left\{\xi^{n} R(\xi ; A)^{n} x ; \xi>\omega, 0 \leqq n / \xi \leqq T\right\}$ is bounded in $X$.

Remark 4.1. Later, we shall treat other two classes of closed operators: One of them is the class of closed operators $A$ in $X$ satisfying conditions $(I ; \omega)$ and

$\left(I I_{\text {exp }} ; k\right)$ there exist numbers $M>0$ and $\omega_{1} \geqq \omega$ such that

$$
\left\|R(\xi ; A)^{n}\right\|_{k} \leqq M\left(\xi-\omega_{1}\right)^{-n} \quad \text { for } \xi>\omega_{1} .
$$

We denote such a class by $\mathscr{B S}_{2}(\omega, k)$. Clearly, $\mathscr{S}_{1}(\omega, k) \supset \mathscr{F}_{2}(\omega, k)$. Another one is the class of closed operators $A$ in $X$ such that

$\left(I_{c} ; \omega\right) \quad\{\lambda \in \mathbf{C} ; \operatorname{Re}(\lambda)>\omega\} \subset \rho(A) ;$

$\left(I I_{\mathrm{pol}} ; k\right)$ there exists a number $M>0$ such that

$$
\|R(\lambda ; A)\| \leqq M(1+|\lambda|)^{k} \quad \text { for } \operatorname{Re}(\lambda)>\omega .
$$

We denote this class by $\mathbb{S S}_{3}(\omega, k)$. Note that condition $\left(I I_{\mathrm{pol}} ; k\right)$ is equivalent to

$\left(I I_{\text {pol }} ; k\right)^{\prime}$ there is a polynomial $p(\xi)$ of the degree $k \geqq 0$ with nonnegative coefficients such that $\|R(\lambda ; A)\| \leqq p(|\lambda|)$ for $\operatorname{Re}(\lambda)>\omega$.

First, as a direct consequence of Theorem 2.3, Lemma 2.4 and Theorem 2.8, we obtain the following:

Theorem 4.2. Let $A \in \mathbb{S}_{1}(\omega, k)$. Then for $m=2 k+1$ there is a uniquely determined one-parameter family $\left\{U_{t} ; t \geqq 0\right\} \subset \mathfrak{B}\left(D\left(A^{m}\right), X\right)$ such 
that

(a) for every $x \in D\left(A^{m}\right)$ and $t \geqq 0, U_{t} x=\lim _{h \rightarrow+0}(I-h A)^{-[t / h]} x$ exists and the convergence is uniform with respect to $t$ in every finite interval;

(b) for every $T>0,\left\|U_{t} x\right\| \leqq M(T)\|x\|_{k}$ for $t \in[0, T]$ and $x \in D\left(A^{m}\right)$, where $M(T)$ is the number given in condition $(I I ; k)$;

(c) for every integer $l \geqq 1$, each $U_{t}$ maps $D\left(A^{m+l}\right)$ into $D\left(A^{l}\right)$ and $A^{l} U_{t} x=U_{t} A^{l} x$ for $t \geqq 0$ and $x \in D\left(A^{m+l}\right)$;

(d) for every $x \in D\left(A^{2 m}\right)$ and $t, s \geqq 0, U_{t+s} x=U_{t} U_{s} x$;

(e) for every $T>0, l \in \mathbf{Z}_{+}$and $x \in D\left(A^{m+1+l}\right)$, there exists a positive number $\beta_{x, l, T}$ such that $\left\|U_{t} x-U_{s} x\right\|_{l} \leqq \beta_{x, l, T}|t-s|$ provided $t, s \in[0, T]$; and furthermore, $U_{t} x-x=\int_{0}^{t} U_{s} A x d s$ for $t \geqq 0$ and $x \in D\left(A^{m+1}\right)$.

If, in addition, $A$ is densely defined, then the assertions mentioned above hold for $m=k$.

Theorem 4.3. Let $A \in \mathscr{S}_{2}(\omega, k)$. Then for $m=2 k+1$ there exists a uniquely determined one-parameter family $\left\{U_{t} ; t \geqq 0\right\} \subset \mathfrak{B}\left(D\left(A^{m}\right), X\right)$ with the properties (a)-(e) stated in Theorem 4.2 and furthermore

(b) ${ }^{\prime} \quad\left\|U_{t} x\right\| \leqq M e^{\omega_{1} t}\|x\|_{k} \quad$ for $t \geqq 0$ and $x \in D\left(A^{m}\right)$;

(f) $\quad R(\lambda ; A) x=\int_{0}^{\infty} e^{-\lambda t} U_{t} x d t \quad$ for $\lambda \in \rho(A)$ with $\operatorname{Re}(\lambda)>\omega_{1}$ and $x \in D\left(A^{m}\right)$.

If, in addition, $\overline{D(A)}=X$, then the assertions mentioned above hold for $m=k$.

Proof. In view of $\left(I I_{\exp } ; k\right)$, Theorem 4.2 yields a unique oneparameter family $\left\{U_{t} ; t \geqq 0\right\} \subset \mathfrak{B}\left(D\left(A^{m}\right), X\right)$ with the properties (a)-(e) stated in Theorem 4.2. Hence, (b) follows from $\left(I I_{\text {exp }} ; k\right)$ and the convergence (a). We then show (f). Let $\lambda \in \rho(A), \operatorname{Re}(\lambda)>\omega_{1}$ and $x \in D\left(A^{m}\right)$, then we can put $y=R(\lambda ; A) x \in D\left(A^{m+1}\right)$. So that (e) implies that $(d / d t)$ $U_{t} y=A U_{t} y=U_{t} A y$, and also $(d / d t)\left[e^{-\lambda t} U_{t} y\right]=-\lambda e^{-\lambda t} U_{t} y+e^{-\lambda t} A U_{t} y=$ $-e^{-\lambda t} U_{t}(\lambda-A) y=-e^{-\lambda t} U_{t} x$. But, $\int_{0}^{T}(d / d t) e^{-\lambda t} U_{t} y d t=\left.e^{-\lambda t} U_{t} y\right|_{t=0} ^{t=T}=$ $e^{-\lambda T} U_{T} y-R(\lambda ; A) x$. Therefore, we have

$$
R(\lambda ; A) x-\int_{0}^{T} e^{-\lambda t} U_{t} x d t=e^{-\lambda T} U_{T} y .
$$


Consequently, using (b)' we have that

$$
\left\|R(\lambda ; A) x-\int_{0}^{T} e^{-\lambda t} U_{t} x d t\right\|=e^{-\operatorname{Re}(\lambda) T}\left\|U_{T} y\right\| \leqq M e^{-\left(\operatorname{Re}(\lambda)-\omega_{1}\right) T}\|y\|_{k}
$$

Hence, we have the assertion (f). The unicity of such a family $\left\{U_{t} ; t \geqq 0\right\}$ follows from (f). In fact, if there is another family $\left\{V_{t} ; t \geqq 0\right\} \subset \mathfrak{B}\left(D\left(A^{m}\right), X\right)$ with the properties (a)-(f), then, $R(\xi, A) x=\int_{0}^{\infty} e^{-\xi t} U_{t} x d t=\int_{0}^{\infty} e^{-\xi t} V_{t} x d t$ for $\xi>\omega_{1}$ and $x \in D\left(A^{m}\right)$. Thus, it follows that $U_{t} x=V_{t} x$ for $t>0$ and $x \in D\left(A^{m}\right)$. The last assertion follows from Lemma 2.7 .

Q.E.D.

Remark 4.4. Let $A$ be a densely defined operator belonging to $\mathbb{S}_{1}(\omega, k)$ and $\left\{U_{t}\right\} \subset \mathfrak{B}\left(D\left(A^{k}\right), X\right)$ be the one-parameter family obtained by Theorem 4.2. Let $\lambda_{0}>\omega$ and then set $H_{t}=U_{t} R\left(\lambda_{0} ; A\right)^{2 k}$ for $t \geqq 0$. Then $H_{t} \in \mathfrak{B}(X)$, $H_{t} H_{s}=H_{s} H_{t}=H_{t+s} H_{0}$ for $t, s \geqq 0$, and $H_{t} x$ is strongly continuous in $t \geqq 0$ for $x \in X$. Also, since $\overline{D\left(A^{n}\right)}=X$ for each $n$ by Lemma $2.7, \overline{R\left(H_{0}^{n}\right)}=X$ for each $n \in \mathbf{Z}_{+}$. Thus, $\left\{H_{t} ; t \geqq 0\right\}$ forms an $R$-semigroup in the sense of $\mathrm{Da}$ Prato [4]. If $A$ is a densely defined operator belonging to $\mathbb{S}_{2}(\omega, k)$ and $\left\{U_{t}\right\}$ is the corresponding one-parameter family obtained by Theorem 4.3, then $\left\|H_{t} x\right\|=\left\|U_{t} R\left(\lambda_{0} ; A\right)^{2 k} x\right\| \leqq M e^{\omega_{1} t}\left\|\left.R\left(\lambda_{0} ; A\right)^{2 k} x\right|_{i k} \leqq M_{1} e^{\omega_{1} t}\right\| x \|$ for some $M_{1}>0$ and $\omega_{1} \geqq \omega$; and hence $\left\{H_{t}\right\}$ becomes an $R$-semigroup of exponential growth.

In each case, $A$ is the infinitesimal generator of the corresponding $R$ semigroup. In fact, if $k=0$, then $H_{t}=U_{t}, t \geqq 0$, form a $\left(C_{0}\right)$-semigroup and $A$ is the infinitesimal generator. If $k \geqq 1$, then $2 k \geqq k+1$, and so it follows from Theorem 4.2 (e) that $U_{t} R\left(\lambda_{0} ; A\right)^{2 k} x$ is strongly continuously differentiable in $t \geqq 0$ for every $x \in X$. Thus, $H_{0}^{\prime} x=A R\left(\lambda_{0} ; A\right)^{2 k} x$ for all $x \in X$. But, noting that $H_{0}^{-1}=\left(\lambda_{0}-A\right)^{2 k}$, we see that $D\left(H_{0}^{-1} H_{0}^{\prime}\right)=D(A)$ and $H_{0}^{-1} H_{0}^{\prime} x=A x$ for $x \in D(A)$.

Lemma 4.5. Let $A \in \mathbb{S S}_{2}(\omega, k)$. If $\overline{D(A)}=X$ and if $\left(I I_{\exp } ; k\right)$ is satisfied for some $M>0$ and $\omega_{1} \geqq \omega$, then $\left(I_{c} ; \omega_{1}\right)$ is satisfied.

Proof. Let $\left\{U_{t} ; t \geqq 0\right\} \subset \mathfrak{B}\left(D\left(A^{k}\right), X\right)$ be the one-parameter family obtained by Theorem 4.3. Let $x \in D\left(A^{k+1}\right)$ and $\operatorname{Re}(\lambda)>\omega_{1}$, then we have that $(\lambda-A) U_{t} x=U_{t}(\lambda-A) x$ and $-(d / d t)\left[e^{-\lambda t} U_{t} x\right]=e^{-\lambda t}(\lambda-A) U_{t} x$. Hence, 


$$
\begin{aligned}
x & =-\int_{0}^{\infty}(d / d t)\left[e^{-\lambda t} U_{t} x\right] d t=\int_{0}^{\infty} e^{-\lambda t}(\lambda-A) U_{t} x d t \\
& =(\lambda-A) \int_{0}^{\infty} e^{-\lambda t} U_{t} x d t \in R(\lambda-A) .
\end{aligned}
$$

Since $D\left(A^{k+1}\right)$ is dense in $X$, it follows that $\overline{R(\lambda-A)}=X$. Now, let $\operatorname{Re}(\lambda)$ $>\xi_{0}>\omega_{1}$ and $v=(\lambda-A) x\left(\in D\left(A^{k}\right)\right)$, then

$$
\begin{aligned}
x & =\int_{0}^{\infty} e^{-\lambda t} U_{t} v d t=\int_{0}^{\infty} e^{-\lambda t}\left(\xi_{0}-A\right)^{k} U_{t}\left(\xi_{0}-A\right)^{-k} v d t \\
& =\int_{0}^{\infty} e^{-\lambda t} e^{\xi_{0} t}(-1)^{k}(d / d t)^{k} e^{-\xi_{0} t} U_{t}\left(\xi_{0}-A\right)^{-k} v d t \\
& =\sum_{l=0}^{k-1}\left(\xi_{0}-\lambda\right)^{l} R\left(\xi_{0} ; A\right)^{l+1} v+\left(\xi_{0}-\lambda\right)^{k} \int_{0}^{\infty} e^{-\lambda t} U_{t} R\left(\xi_{0} ; A\right)^{k} v d t .
\end{aligned}
$$

Therefore,

$$
\begin{aligned}
\|x\| & \leqq \sum_{l=0}^{k-1}\left|\xi_{0}-\lambda\right|^{l}\left\|R\left(\xi_{0} ; A\right)^{l+1}\right\|\|v\| \\
& +\left|\xi_{0}-\lambda\right|^{k} \int_{0}^{\infty} e^{-\operatorname{Re}(\lambda) t} e^{\omega_{1} t}\left\|e^{-\omega_{1} t} U_{t} R(\xi ; A)^{k}\right\|\|v\| d t \\
& \leqq \operatorname{const}\left(\xi_{0}, \lambda, \omega_{1}, k, M\right)\|(\lambda-A) x\| .
\end{aligned}
$$

Since $x$ was arbitrary in $D\left(A^{k}\right)$ and since $D\left(A^{k}\right)$ is a core of $A$ by Lemma 2.7 , we have that $\|x\| \leqq$ const. $\|(\lambda-A) x\|$ for $x \in D(A)$. This means that $(\lambda-A)^{-1}$ is defined as a closed operator with domain $R(\lambda-A)$ and $\left\|(\lambda-A)^{-1} x\right\| \leqq$ const. $\|x\|$ for $x \in R(\lambda-A)$. But, since $\overline{R(\lambda-A)}=X$, it follows that $(\lambda-A)^{-1} \in \mathfrak{B}(X)$, that is, $\lambda \in \rho(A)$. Therefore, $\{\lambda \in \mathbb{C} ; \operatorname{Re}(\lambda)$ $\left.>\omega_{1}\right\} \subset \rho(A)$.

Q.E.D.

Consequently, we obtain the following result which is an extension of the generation theorem of $\left(C_{0}\right)$-semigroups in the sense that $\left\{\xi^{n} R(\xi ; A)^{n}\right\}$ is equicontinuous from a Banach space $\left[D\left(A^{k}\right)\right]$ into $X$; the result for $k=0$ gives the generation theorem of $\left(C_{0}\right)$-semigroups.

Theorem 4.6. Let $A$ be a densely defined operator belonging to $\mathbb{S}_{2}$ $(\omega, k)$. Then $\left(I_{c} ; \omega_{1}\right)$ is satisfied for some $\omega_{1} \geqq \omega$ and there is a uniquely determined one-parameter family $\left\{U_{t} ; t \geqq 0\right\} \subset \mathfrak{B}\left(D\left(A^{k}\right), X\right)$ with the properties (a)-(f) stated in Theorem 4.3 for $m=k$ and furthermore

$$
\text { (f) } \quad R(\lambda ; A) x=\int_{0}^{\infty} e^{-\lambda t} U_{t} x d t \quad \text { for } \operatorname{Re}(\lambda)>\omega_{1} \text { and } x \in D\left(A^{k}\right) \text {; }
$$


(g) $\left\|R(\lambda ; A)^{n} x\right\| \leqq\left(\operatorname{Re}(\lambda)-\omega_{1}\right)^{-n} M\|x\|_{k}$ for $\operatorname{Re}(\lambda)>\omega_{1}$ and $x \in D\left(A^{k}\right)$.

Proof. Assume that $A$ satisfies $\left(I I_{\exp } ; k\right)$ for some $M>0$ and $\omega_{1} \geqq \omega$, then from Lemma 4.5 it follows that $A$ satisfies $\left(I_{c} ; \omega_{1}\right)$. Therefore, (f) is obtained from Theorem 4.3 ( $\mathrm{f})$. (g) is proved as follows: Since the resolvent equation yields that

$$
\begin{gathered}
R(\lambda ; A)^{n} x=(n-1) !^{-1} \int_{0}^{\infty} t^{n-1} e^{-\lambda t} U_{t} x d t, \quad \text { for } \operatorname{Re}(\lambda)>\omega_{1}, \\
\left\|R(\lambda ; A)^{n} x\right\| \leqq(n-1) !^{-1} M\|x\|_{k} \int_{0}^{\infty} t^{n-1} e^{-\left(\operatorname{Re}(\lambda)-\omega_{1}\right) t} d t \leqq\left(\operatorname{Re}(\lambda)-\omega_{1}\right)^{-n} M\|x\|_{k}
\end{gathered}
$$

Next, for an $\omega \in \mathbb{R}$ and a $k \in \mathbb{Z}_{+}$, let us consider class $\mathcal{S}_{3}(\omega, k)$, the class of closed operators $A$ in $X$ satisfying $\left(I_{c} ; \omega\right)$ and $\left(I I_{\mathrm{pol}} ; k\right)$ stated in Remark 4.1.

The relations among classes $\mathscr{B S}_{2}(\omega, k)$ and $\mathscr{B S}_{3}(\omega, k)$ can be stated as follows:

Theorem 4.7. Let $\omega \in \mathbb{R}$ and $k \in \mathbb{Z}_{+}$. Then we have:

(a) Let $\gamma>\max \{0, \omega\}$. Then $\mathbb{S}_{3}(\omega, k) \subset \mathbb{S}_{2}(\gamma, k+2)$.

(b) Conversely, let $A \in \mathbb{B}_{2}(\omega, k)$. If $A$ satisfies condition $\left(I_{c} ; \omega\right)$, then $A \in \mathbb{F}_{3}(\omega, 2 k+1)$. If $A$ is densely defined, then $A \in \mathcal{F}_{3}\left(\omega_{1}, k\right)$ for some $\omega_{1} \geqq \omega$.

Proof. (a) Let $A \in \mathbb{S}_{3}(\omega, k)$. If $x \in D\left(A^{k+2}\right)$, then we have

$$
R(\lambda ; A) x=\lambda^{-1} x+\lambda^{-2} A x+\cdots+\lambda^{-k-2} A^{k+1} x+\lambda^{-k-2} R(\lambda ; A) A^{k+2} x
$$

Let $\max \{0, \omega\}<\gamma^{\prime}<\gamma$, then by the calculation of residues and $\left(I I_{\mathrm{pol}} ; k\right)$,

$$
\left\{\begin{aligned}
& y(t ; x) \lim (2 \pi i)^{-1} \int_{\gamma^{\prime}-i \tau}^{\gamma^{\prime}+i_{\tau}} e^{\lambda t} R(\lambda ; A) x d \lambda \\
= & \sum_{j=1}^{k+2}(2 \pi i)^{-1} \int_{\gamma^{\prime}-i \infty}^{\gamma^{\prime}+i \infty} e^{\lambda t} \lambda^{-j} d \lambda A^{j-1} x \\
+ & (2 \pi i)^{-1} \int_{\gamma^{\prime}-i \infty}^{\gamma^{\prime}+i \infty} e^{\lambda t} R(\lambda ; A) A^{k+2} x \lambda^{-k-2} d \lambda \\
= & \sum_{j=1}^{k+2} \frac{t^{j-1}}{(j-1) !} A^{j-1} x+(2 \pi i)^{-1} \int_{\gamma^{\prime}-i \infty}^{\gamma^{\prime}+i \infty} e^{\lambda t} R(\lambda ; A) A^{k+2} x \lambda^{-k-2} d \lambda
\end{aligned}\right.
$$

By $\left(I I_{\text {pol }} ; k\right)$, the integrand of the above right side is estimated by 
$M^{\prime} e^{\gamma^{\prime} t}\|x\|_{k+2}$ for $\lambda$ sufficiently large and some $M^{\prime}>0$. Hence, it follows that there is a sufficiently large positive number $M$ such that $\|y(t ; x)\|$ $\leqq M e^{\gamma^{\prime} t}\|x\|_{k+2}$. Also, by the contour integration argument we see that the integral of the right side is 0 in case $t=0$, and so $y(0 ; x)=x$. Hence, $\int_{0}^{\infty} e^{-\lambda t} y(t ; x) d t$ is absolutely convergent for $\lambda$ with $\operatorname{Re}(\lambda)>\gamma^{\prime}$ and

$$
\begin{aligned}
& \int_{0}^{\infty} e^{-\lambda t} y(t ; x) d t=\sum_{j=1}^{k+2} \lambda^{-j} A^{j-1} x+(2 \pi i)^{-1} \int_{0}^{\infty} e^{-\lambda t} \int_{\gamma^{\prime}-i \infty}^{\gamma^{\prime+i \infty}} e^{\mu t} R(\mu ; A) A^{k+2} x \mu^{-k-2} \\
& d \mu d t \\
& =\sum_{j=1}^{k+2} \lambda^{-j} A^{j-1} x+(2 \pi i)^{-1} \int_{\gamma^{\prime}-i \infty}^{\gamma^{\prime}+i \infty}(\lambda-\mu)^{-1} R(\mu ; A) A^{k+2} x \mu^{-k-2} d \mu=R(\lambda ; A) x .
\end{aligned}
$$

Therefore, the resolvent equation yields that

$$
R(\lambda ; A)^{n} x=(n-1) !^{-1} \int_{0}^{\infty} t^{n-1} e^{-\lambda t} y(t ; x) d t .
$$

But, since $\sup \left\{\left\|e^{-\gamma^{\prime} t} y(t ; x)\right\| ; t \geqq 0\right\} \leqq M\|x\|_{k+2}$, we have

$$
\left\|R(\lambda ; A)^{n} x\right\| \leqq M\|x\|_{k+2}(n-1) !^{-1} \int_{0}^{\infty} t^{n-1} e^{-\left(\lambda-\gamma^{\prime}\right) t} d t \leqq\left(\lambda-\gamma^{\prime}\right)^{-n} M\|x\|_{k+2}
$$

for $\lambda>\gamma^{\prime}$. This means that $(I ; \gamma)$ and $\left(I I_{\exp } ; k+2\right)$ are satisfied. Therefore, $A \in \mathbb{S B}_{2}(\gamma, k+2)$.

(b) First, we assume that $A$ satisfies conditions $\left(I_{c} ; \omega\right)$ and $\left(I I_{\exp } ; k\right)$ for some $\omega_{1} \geqq \omega$ and $M>0$. Let $m=2 k+1, \lambda \in \rho(A)$ and $\lambda_{0}$ be a fixed number with $\operatorname{Re}\left(\lambda_{0}\right)>\omega$. Then by the resolvent equation we can write as

$$
R(\lambda ; A)=\sum_{i=0}^{m-1}\left(\lambda_{0}-\lambda\right)^{i} R\left(\lambda_{0} ; A\right)^{i+1}+\left(\lambda_{0}-\lambda\right)^{m} R(\lambda ; A) R\left(\lambda_{0} ; A\right)^{m} .
$$

On the other hand, we see from Theorem 4.3 that $R(\lambda ; A) x=\int_{0}^{\infty} e^{-\lambda t} U_{t} x d t$ for $\lambda$ with $\operatorname{Re}(\lambda)>\omega_{1}$ and $x \in D\left(A^{m}\right)$. Thus,

$$
R(\lambda ; A) R\left(\lambda_{0} ; A\right)^{m} x=\int_{0}^{\infty} e^{-\lambda t} U_{t} R\left(\lambda_{0} ; A\right)^{m} x d t, \quad \text { for } \operatorname{Re}(\lambda)>\omega_{1} \text { and } x \in X .
$$

Also, we have using Theorem 4.3 (b) that

$$
\left\|U_{t} R\left(\lambda_{0} ; A\right)^{m} x\right\|_{1} \leqq M e^{\omega_{1} t}\left\|R\left(\lambda_{0} ; A\right)^{m} x\right\|_{m} \leqq M e^{\omega_{1} t}\left\{\sum_{j=0}^{m}\left\|A^{j} R\left(\lambda_{0} ; A\right)^{m}\right\|\right\}\|x\| .
$$

Therefore, letting $\varepsilon>0$,

$$
\sup _{\operatorname{Re}(\lambda)>\omega_{1}+\varepsilon} \int_{0}^{\infty} \mid ! e^{-\lambda t} U_{t} R\left(\lambda_{0} ; A\right)^{m} \| d t=C\left(\lambda_{0}\right)<+\infty .
$$


Hence, $\|R(\lambda ; A)\| \leqq \sum_{i=1}^{m-1}\left|\lambda-\lambda_{0}\right|{ }^{i}\left\|R\left(\lambda_{0} ; A\right)^{i+1}\right\|+\left|\lambda-\lambda_{0}\right|{ }^{m} C\left(\lambda_{0}\right)$. This means that $\left(I I_{\mathrm{pol}} ; 2 k+1\right)$ is satisfied.

Next, assume that $A$ is a densely defined, closed operator satisfying $(I ; \omega)$ and $\left(I I_{\exp } ; k\right)$ for some $\omega_{1} \geqq \omega$ and $M>0$. Then, in view of Lemma $4.5,\left(I_{c} ; \omega_{1}\right)$ is satisfied. Hence, letting $m=k$ and repeating the same argument as above, we see that $A \in \mathcal{B S}_{3}\left(\omega_{1} ; k\right)$.

Q.E.D.

Remark 4.8. The complete infinitesimal generator of an $(A)$-semigroup of the type $\omega_{0}$ belongs to $\mathcal{S}_{3}\left(\omega_{1} ; 0\right)$ for some $\omega_{1}>\omega_{0}$. The complete infinitesimal generator of either $(0, A)$ - or $(1, A)$-semigroup of the type $\omega_{0}$ belongs to $\mathscr{S}_{2}\left(\omega_{0} ; 1\right)$. Furthermore, the infinitesimal generator of a $\left(C_{0}\right)$ semigroup of the type $\omega_{0}$ belongs to $\mathbb{S}_{2}\left(\omega_{0}, 0\right)$.

Proof. Assume that $A$ is the complete infinitesimal generator of an (A)-semigroup $\left\{T_{t}\right\}$ of type $\omega_{0}$; then by condition (A.2) stated in Section 1 there is an $\omega_{1}>\omega_{0}$ such that $\rho(A) \supset\left\{\lambda \in \mathbf{C} ; \operatorname{Re}(\lambda)>\omega_{1}\right\}$ and such that $\sup \left\{\|R(\lambda ; A)\| ; \operatorname{Re}(\lambda)>\omega_{1}\right\}<+\infty$. This means that $A \in \mathcal{B}_{3}\left(\omega_{1}, 0\right)$. Next, let $A$ be the complete infinitesimal generator of a $(0, A)$ - or $(1, A)$ semigroup $\left\{T_{t}\right\}$ of type $\omega_{0}$, then $N(x)=\sup \left\{\left\|e^{-\omega_{0} t} T_{t} x\right\| ; t>0\right\}<+\infty$ for each $x \in D(A)$ and $R(\lambda ; A) x=\int_{0}^{\infty} e^{-\lambda t} T_{t} x d t$ for $\operatorname{Re}(\lambda)>\omega_{0}$ and $x \in X$. Hence, $\sup _{t>0}\left\|e^{-\omega_{0} t} T_{t}\right\|_{1}=M<+\infty$ and hence by the same way as in the estimate of Theorem 4.7 (a), we see that $\left\|R(\lambda ; A)^{n} x\right\| \leqq M\|x\|_{1} /\left(\lambda-\omega_{0}\right)^{n}$. Therefore, $A \in \mathcal{S}_{2}\left(\omega_{0}, 1\right)$. Finally, it is well-known that a closed operator $A$ is the infinitesimal generator of a $\left(C_{0}\right)$-semigroup if and only if $A \in \mathscr{B}_{2}\left(\omega_{0}, 0\right)$ by Hille-Yosida-Miyadera-Phillips' theorem.

Remark 4.9. The following are well-known (see for example Krein [11]):

(a) If a closed operator $A$ satisfies $\left(I_{c} ; \omega\right)$ and $\left(I I_{\mathrm{pol}} ; k\right)$ for some $\omega$ and $k=-1$, then $A$ generates a holomorphic semigroup of class $\left(C_{0}\right)$; so $A \in \mathbb{B}_{2}\left(\omega_{0}, 0\right)$.

(b) If a closed operator $A$ satisfies $\left(I_{c} ; \omega\right)$ and $\left(I I_{\text {pol }} ; k\right)$ for some $\omega$ and $k$ with $-1<k<1 / 2$, then $A$ generates a $(1, A)$-semigroup $\left\{T_{t} ; t>0\right\}$ such that for each $x \in X, T_{t} x$ is of $C^{\infty}$ for $t>0$.

Finally, we consider some examples. Let us consider the system of partial differential equations 


$$
(\partial / \partial t) u(t, s)=P(D) u(t, s),(t, s) \in \overline{\mathbf{R}}_{+} \times \mathbb{R},
$$

where $P(\xi)$ is an $m \times m$-matrix of polynomials $p_{i j}(\xi)$ of $\xi$ with complex constant coefficients and $P(D)$ is defined by substituting $D=i \partial / \partial s$ into $\xi$, and $u(t, s)$ represents an $m$-vector of numerical functions $u_{i}(t, s)$ of two variables $(t, s)$. The Cauchy problem for the equation (4.4) is the problem to find the solution $u(t, s)$ of (4.4) associated with the given initial condition

$$
u(0, s)=u_{0}(s), \quad s \in \mathbf{R} .
$$

Now, we consider this problem over the space $\overbrace{L_{2}=L_{2}(\mathbf{R}) \times L_{2}(\mathbf{R}) \cdots \times L_{2}(\mathbf{R})}^{m}$ with the inner product

$$
<\phi, \psi>=\int_{-\infty}^{\infty} \sum_{i=1}^{m} \phi_{i}(s) \overline{\phi_{i}(s)} d s, \quad \phi, \phi \in L_{2} .
$$

Then, applying the Fourier transform, (4.4) is reduced to the following Cauchy problem for a system of ordinary differential equations with $\xi$ as a parameter:

$$
\begin{array}{ll}
(d / d t) \hat{u}(t, \xi)=P(\xi) \hat{u}(t, \xi), & t \in \overline{\mathbf{R}}_{+}, \\
\hat{u}(0, \xi)=\hat{u}_{0}(\xi), & \xi \in \mathbf{R},
\end{array}
$$

where $P(\xi)$ is the same matrix of polynomials and $\hat{u}_{0}(\xi)$ is the Fourier transform of $u_{0}(s) . P(\xi), \xi \in \mathbb{R}$, define an unbounded operator $P$ of multiplication in $L_{2}$ by the relation

$$
[P \phi](\xi)=P(\xi) \phi(\xi), \quad \xi \in \mathbb{R},
$$

where $[P \phi](\xi)$ means a representative function of $P \phi \in L_{2}$. The domain $D(P)$ of $P$ is regarded as the class of elements $\phi \in L_{2}$ such that $P \phi \in L_{2}$. It is well-known that $P(D)$ can be regarded as a closed operator in $L_{2}$ and $C_{0}^{\infty}$ can be regarded as a core of $P(D)$ in a natural way. We consider $A=P(D)$ as an example of the closed operator treated in this paper. Hence, in view of the Fourier-Plancherel theorem, it is sufficient to esti- 
mate the iterations of the resolvent of $P(\xi)$, in order to estimate those of $P(D)$.

The solution of the system (4.6) with the initial condition (4.7) is given by

$$
u(t ; \xi)=e^{t P(\xi)} \hat{u}_{0}(\xi), \quad \xi \in \mathbf{R} ;
$$

we denote by $\left[U_{t} u_{0}\right](s)$ the inverse Fourier transform of $e^{t P(\xi)} \hat{u}_{0}(\xi)$ if it makes sense.

When we consider examples in this paper, we consider unbounded operators $P$ of multiplication, defined in $L_{2}$ by $(4.8)$, such that

$$
P(\xi)=p E+q F, p=p(\xi), q=q(\xi),
$$

where $E$ denotes an $m \times m$-unit matrix and $F$ denotes an $m \times m$-nilpotent matrix such that only upper off-diagonal elements are 1 , and $p$ and $q$ are polynomials of $\xi$ with complex coefficients; hence each $P(\xi)$ is an upper bi-diagonal complex $m \times m$-matrix. For each $\xi$, the eigen-values of $P(\xi)$ are same and equal to $p(\xi)$, and so $\rho(P)$ is contained in the complement of $\sigma(P)=\{p(\xi) ; \xi \in \mathbb{R}\}$. By simple calculations, we have

$$
(\lambda-P(\xi))^{-1}=\sum_{j=0}^{m-1}(\lambda-p)^{-j-1} q^{j} F^{j},
$$

where $F^{0}=E$. Hence, if

$$
m \operatorname{deg}(p) \geqq(m-1) \operatorname{deg}(q)
$$

and $\lambda \notin \sigma(P),(\lambda-P(\xi))^{-1}, \xi \in \mathbb{R}$, define a bounded operator $(\lambda-P)^{-1}$ on $L_{2}$ by the relation

$$
\left[(\lambda-P)^{-1} \phi\right](\xi)=(\lambda-P(\xi))^{-1} \phi(\xi), \quad \phi \in L_{2},
$$

where $\left[(\lambda-P)^{-1} \phi\right](\xi)$ means a representative function of $(\lambda-P)^{-1} \phi \in L_{2}$. It is easy to see that $(\lambda-P)^{-1}$ is the resolvent of $P$ at $\lambda$. Also, we have

$$
e^{t P(\xi)}=e^{p t} \sum_{j=0}^{m-1}(j !)^{-1} t^{j} q^{j} F^{j}
$$

For each $t>0, e^{t P(\xi)}, \xi \in \mathbf{R}$, define an operator $e^{t P}$ in $L_{2}$ by the relation 


$$
\left[e^{t P} \phi\right](\xi)=e^{t P(\xi)} \phi(\xi), \quad \phi \in D\left(e^{t P}\right) .
$$

Now, we consider an example. As is seen from the definition, a sequence of classes $\mathscr{S}_{2}(\omega, k), k \in \mathbf{Z}_{+}$, is monotone increasing in the sense of inclusion. By the way, given a $k$ we can find an operator $P$, defined by (4.8), such that $P \in \mathbb{S}_{2}(\omega, k+1) \backslash \mathscr{B}_{1}(\omega, k)$. Hence, each class $\mathbb{S}_{2}(\omega, k)$ is properly contained in class $\mathcal{S S}_{2}(\omega, k+1)$. This also suggests that it will be necessary to consider classes $\mathcal{B S}_{j}(\omega, k), k$ large and $j=1,2,3$, as the number of equations in a system increases.

Example 4.10. Let $k$ be a positive integer and let us consider an $k+1$

unbounded operator $P$ of multiplication, defined in $L_{2}=L_{2}(R) \times \cdots \times L_{2}(R)$ by (4.8), such that $P(\xi)$ is a $(k+1) \times(k+1)$-matrix of the form $(4.10)$ with

$$
p=p(\xi)=i \xi^{d} \text { and } q=q(\xi)=\xi^{r}, \quad \xi \in \mathbf{R},
$$

where $d$ and $r$ are positive integers. The eigenvalue of $P(\xi)$ is $i \xi^{d}$. Hence, if $d(k+1) \geqq r k$ and $\operatorname{Re}(\lambda)>0$, then in view of (4.11), (4.12) and (4.13) the matrices $(\lambda-P(\xi))^{-1}, \xi \in \mathbf{R}$, define a bounded operator $(\lambda-P)^{-1}$ on $L_{2}$ which is the resolvent of $P$ at $\lambda$. We then assume that

$$
d<r \leqq k^{-1}(k+1) d,
$$

note that $r-d \leqq k^{-1} d$.

First we show that under condition (4.16) the matrices $e^{t P(\xi)}, \xi \in \mathbf{R}$, define a bounded operator $e^{t P}$ from $\left[D\left(P^{k+1}\right)\right]$ into $L_{2}$. Let $\phi=\left(\phi_{j}\right) \in D$ $\left(P^{k+1}\right)$. Then, in view of (4.14) for $m=k+1$, we see that $e^{t P} \phi \in L_{2}$ if we can prove that $q \phi_{j}, q^{2} \phi_{j}, \ldots, q^{j-1} \phi_{j} \in L_{2}(R)$ for $1 \leqq j \leqq k+1$. For the proof, it suffices to prove that $(1+|\xi|)^{d j} \phi_{j} \in L_{2}(R)$ for $1 \leqq j \leqq k+1$; if so, we see that $q^{j-1} \phi_{j} \in L_{2}(R)$ for $1 \leqq j \leqq k+1$, since $\operatorname{deg}\left(q^{j-1}\right)=k r+r(j-1-k)$ $\leqq d(k+1)+d(j-1-k)=\operatorname{deg}\left(p^{j}\right)$ by $(4.16)$. Since

$$
P(\xi)^{m}=\sum_{l=0}^{m}{ }_{m} C_{l} p^{m-l} q^{l} F^{l}, \quad 1 \leqq m \leqq k+1,
$$

the $m$-th element of $P^{m} \phi, 1 \leqq m \leqq k+1$, can be written as

$$
\left[P^{m} \phi\right]_{m}=p^{m} \phi_{m}+\sum_{l=1}^{m_{0}}{ }_{m} C_{l} p^{m-l} q^{l} \phi_{m+l}, \quad 1 \leqq m \leqq k+1,
$$


where $m_{0}=\min \{k+1-m, m\}$. First, since $\left[P^{k+1} \phi\right]_{k+1}=p^{k+1} \phi_{k+1} \in L_{2}(R)$, we have that $(1+|\xi|)^{d(k+1)} \phi_{k+1} \in L_{2}(R)$. Also, $\left[P^{k} \phi\right]_{k}=p^{k} \phi_{k}+k p^{k-1} q \phi_{k+1}$ $\in L_{2}(R)$ and $\operatorname{deg}\left(p^{k-1} q\right)=d(k-1)+r=d k+r-d \leqq d(k+1)=\operatorname{deg}\left(p^{k+1}\right)$; hence $p^{k-1} q \phi_{k+1} \in L_{2}(R)$. This implies that $p^{k} \phi_{k} \in L_{2}(R)$, Thus, it follows that $(1+|\xi|)^{d k} \phi_{k} \in L_{2}(R)$. Assume now that $(1+|\xi|)^{d m} \phi_{m} \in L_{2}(R)$ for $j+1 \leqq m \leqq k+1$. Then, in view of $(4.17)^{\prime}$ for $m=j$ and the relation deg $\left(p^{j-l} q^{l}\right)=d(j-l)+r l \leqq d(j+l)=\operatorname{deg}\left(p^{j+l}\right)$ for $1 \leqq l \leqq m_{0}$, we have that $p^{j-l} q^{l} \phi_{j+l} \in L_{2}(R)$, which means that $p^{j} \phi_{j} \in L_{2}(R)$ or $(1+|\xi|)^{d j} \phi_{j} \in L_{2}(R)$. In this manner, we obtain, by induction, that $q^{j-1} \phi_{j} \in L_{2}(R)$ for $1 \leqq j \leqq k$. Also, it is now clear that $q^{l} \phi_{j} \in L_{2}(R)$ for $1 \leqq l \leqq k-1$ and $1 \leqq j \leqq k+1$. Consequently, $e^{t P} \phi \in L_{2}$ for $\phi \in D\left(P^{k+1}\right)$.

By the way, we can show that $e^{t P} \in \mathfrak{B}\left(D\left(P^{k+1}\right), L_{2}\right)$ for $t>0$ as follows: Let $t>0, \phi^{(\nu)} \in D\left(P^{k+1}\right), \phi^{(\nu)} \rightarrow \phi$ in $\left[D\left(P^{k+1}\right)\right]$, and let $e^{t P} \phi^{(\nu)} \rightarrow \psi$ in $L_{2 .}$. Then, since $(q-i)^{-1} \in L_{\infty}(R)$ and $(q-i)^{-(k+1-j)}\left[e^{t P} \phi^{(\nu)}\right]_{j}=$ $e^{p t} \sum_{l=0}^{k+1-j}(l !)^{-1} t^{l}(q-i)^{-(k+1-j)} q^{l} \phi_{j+l}^{(\nu)} \rightarrow(q-i)^{-(k+1-j)} \psi_{j}$ in $L_{2}(R)$. But, $(q-i)^{-(k+1-j)} q^{l} \phi_{j+l}^{(\nu)} \rightarrow(q-i)^{-(k+1-j)} q^{l} \phi_{j+l}$ in $L_{2}(R)$ for $0 \leqq l \leqq k+1-j$; hence it follows that $\psi_{j}=\left[e^{t P} \phi\right] j, 1 \leqq j \leqq k+1$. This means that $e^{t P} \in \mathfrak{S}\left(D\left(P^{k+1}\right)\right.$, $L_{2}$ ) for $t>0$. So, the closed graph theorem implies that $e^{t P} \in \mathfrak{B}\left(D\left(P^{k+1}\right)\right.$, $L_{2}$ ) for $t>0$.

Also, noting that $\left\|\left[e^{t P} \phi\right]_{j}\right\|=\left\|e^{p t} \sum_{l=0}^{k+1-j}(l !)^{-1} t^{l} q^{l} \phi_{j+l}\right\| \leqq e^{t} \max _{l}\left\|q^{l} \phi_{j+l}\right\|$ and employing the resonance theorem, a large number $M$ can be found such that $\left\|e^{-t} e^{t P} \phi\right\| \leqq M\|\phi\|_{k+1}$ for $t>0$. On the other hand, for every $\xi \in \mathbf{R}, n \geqq 1$ and $\phi \in D\left(P^{k+1}\right)$,

$$
(\lambda-P(\xi))^{-n} \phi(\xi)=(n-1) !^{-1} \int_{0}^{\infty} e^{-\lambda t} t^{n-1} e^{t P(\xi)} \phi(\xi) d t .
$$

Hence, by Fubini's theorem

$$
\left\|(\lambda-P)^{-n} \phi\right\| \leqq(n-1) !^{-1} \int_{0}^{\infty} e^{-(\lambda-1) t} t^{n-1} d t M\|\phi\|_{k+1} .
$$

Therefore, from the Fourier-Plancherel theorem it follows that $P(D) \in$ $\mathbb{S}_{2}(1, k+1)$.

Next, we demonstrate that $P(D)$ treated above does not belong to $\left(\mathcal{S}_{1}(\omega, k)\right.$ if $d$ is sufficiently large. Under condition (4.16), assume that

$$
r-d=a+b
$$


where $a$ and $b$ are positive integers, note that $2 \leqq a+b \leqq k^{-1} d \leqq d$. Let

$$
\left\{\begin{array}{l}
\phi_{1}=\phi_{2}=\cdots=\phi_{k-1}=0, \\
\phi_{k}=(1+|\xi|)^{b-r k}\left(i \xi^{r-d}+1\right), \phi_{k+1}=k(1+|\xi|)^{b-r k} .
\end{array}\right.
$$

Then, in view of (4.17), we see that

$$
\left[P^{m} \phi\right]_{j}={ }_{m} C_{k-j} p^{m-(k-j)} q^{k-j} \phi_{k}+{ }_{m} C_{k+1-j} p^{m-(k+1-j)} q^{k+1-j} \phi_{k+1},
$$

for $1 \leqq j \leqq k+1$ and $m=k, k+1$. If $m=k$ and $2 \leqq j \leqq k+1$, then $\operatorname{deg}\left(p^{j} q^{k-j}\left(i \xi^{r-d}+1\right)\right)=\operatorname{deg}\left(p^{j-1} q^{k+1-j}\right)=r k+(d-r)(j-1) \leqq r k-b-a$, and so, it follows that $\left[P^{k} \phi\right]_{j} \in L_{2}(R)$ for $2 \leqq j \leqq k+1$. On the other hand,

$$
\begin{aligned}
{\left[P^{k} \phi\right]_{1} } & =k p q^{k-1} \phi_{k}+q^{k} \phi_{k+1}=k q^{k-1}(1+|\xi|)^{b-r k}\left(i \xi^{d}\left(i \xi^{r-d}+1\right)+\xi^{r}\right) \\
& =k p q^{k-1}(1+|\xi|)^{b-r k} \in L_{2}(R),
\end{aligned}
$$

and

$$
\begin{aligned}
& {\left[P^{k+1} \phi\right]_{1}=2^{-1}(k+1) k p^{2} q^{k-1} \phi_{k}+(k+1) p q^{k} \phi_{k+1} } \\
= & (k+1) p q^{k-1}(1+|\xi|)^{b-r k}\left(\left(1-2^{-1} k\right) q+2^{-1} k p\right) \notin L_{2}(R),
\end{aligned}
$$

note here that $\operatorname{deg}\left(p^{2} q^{k-1}\right)=r k+d+(d-r)=r k-b+d-a \geqq(r k-b)+b$. Consequently, we see that $\phi \in D\left(P^{k}\right) \backslash D\left(P^{k+1}\right)$.

Now, in view of (4.14), we have

$$
\left[e^{t P} \phi\right]_{1}=(k-1) !^{-1} e^{p t}\left[t^{k-1} q^{k-1} \phi_{k}+k^{-1} t^{k} q^{k} \phi_{k+1}\right] \text {. }
$$

Hence,

$$
\begin{aligned}
& \left\|(\lambda-P)^{-n} \phi\right\|^{2}=(n-1) !^{-2}\left\|\int_{0}^{\infty} e^{-\lambda t} t^{n-1} e^{t P} \phi d t\right\|^{2} \\
& \geqq(n-1) !^{-2}\left\|\int_{0}^{\infty} e^{-\lambda t} t^{n-1}\left[e^{t P} \phi\right]_{1} d t\right\|^{2} \\
& \geqq(k-1) !^{-2}\left\{(n-1) !^{-2} 2^{-1} k^{-2}\left\|\int_{0}^{\infty} e^{-\lambda t} e^{p t} t^{n+k-1} d t q^{k} \phi_{k+1}\right\|^{2}\right. \\
& \left.\quad-(n-1) !^{-2}\left\|\int_{0}^{\infty} e^{-\lambda t} e^{p t} t^{r+k-2} d t q^{k-1} \phi_{k}\right\|^{2}\right\} \\
& \equiv(k-1) !^{-2}\left\{2^{-1} k^{-2} J_{1}-J_{2}\right\},
\end{aligned}
$$


where we used the relation $\|x+y\|^{2} \geqq 2^{-1}\|x\|^{2}-\|y\|^{2}$. Now,

$$
\begin{aligned}
& J_{1}=((n+k-1) ! /(n-1) !)^{2}\left\|(\lambda-p)^{-(n+k)} q^{k} \phi_{k+1}\right\|^{2} \\
& \geqq \lambda^{-2 n}(n / \lambda)^{2 k}\left\|(1-p / \lambda)^{-(n+k)} q^{k} \phi_{k+1}\right\|^{2} \\
& \geqq \lambda^{-2 n}(n / \lambda)^{2 k} \int_{-\gamma}^{\gamma}\left|(1-p / \lambda)^{-(n+k)}\right|^{2}\left|q^{k} \phi_{k+1}\right|^{2} d \xi \quad \text { for every } \gamma>0,
\end{aligned}
$$

and

$$
\begin{aligned}
& J_{2} \leqq(n-1) !^{-2} \int_{-\infty}^{\infty}\left(\int_{0}^{\infty} e^{-\lambda t} t^{n+k-2} d t\right)^{2}\left|q^{k-1} \phi_{k}\right|^{2} d \xi \\
& =\left((n+k-2) ! /(n-1) ! \lambda^{n+k-1}\right)^{2}\left\|q^{k-1} \phi_{k}\right\|^{2} \\
& \leqq \lambda^{-2 n}((n+k) / \lambda)^{2(k-1)}\left\|q^{k-1} \phi_{k}\right\|^{2} .
\end{aligned}
$$

Since $(1-p / \lambda)^{-n-k} \rightarrow e^{p t}$ as $\lambda \rightarrow+\infty$ and $n / \lambda \rightarrow t>0$, uniformly for $\xi \in$ $[-\gamma, \gamma]$, we see that

$$
\begin{aligned}
& \lim _{\substack{\lambda \rightarrow+\infty \\
n / \lambda \rightarrow t}}\left\|\lambda^{n}(\lambda-P)^{-n} \phi\right\|^{2} \geqq(k-1) !^{-2}\left\{2^{-1} k^{-2} t^{2 k} \int_{-\gamma}^{\gamma}\left|q^{k} \phi_{k+1}\right|^{2} d \xi-t^{2 k-2}\left\|q^{k-1} \phi_{k}\right\|^{2}\right\} \\
& \text { for every } \gamma>0 \text {. This implies that } P(D) \notin \mathbb{S}_{1}(1, k) .
\end{aligned}
$$

Remark 4.11. Let $P(D)$ be the differential operator mentioned above. We have observed in Example 4.10 that $P(D) \in \mathbb{S}_{2}(1, k+1)$. Hence, the solution operator $\left\{U_{t}\right\}$ of $A C P_{1}$ for $P(D)$ can be constructed so well as $\left\{U_{t}\right\} \subset \mathfrak{B}\left(D\left(P^{k+1}\right), L_{2}\right)$. Since $U_{t}, t>0$, can not be extended to any bounded operators $T_{t}$ on $L_{2},\left\{U_{t}\right\}$ may be called the unbounded solution operator, so far as the problem is treated in the original $L_{2}$. By the way, as will be seen in the next section, a densely defined, closed operator $A$ in a Banach space $X$ is the infinitesimal generator of an E.D.S.G. $T$ if and only if $A$ belongs to class $\mathbb{G}_{2}(\omega, k)$ for some $\omega \in \mathbf{R}$ and $k \in \mathbf{Z}_{+}$and, in this case, the corresponding solution operator $\left\{U_{t}\right\}$ is related to the E.D.S.G. $T$ in the sense of the relation (5.1). Hence, the $P(D)$ is the infinitesimal generator of an E.D.S.G. Also, examples treated in the theory of distribution semigroup can be regarded as the examples for our argument. In this manner, the results in this section will be sometimes applicable, when we consider the system of partial differential equations or 
the partial differential equation of higher order.

Finally, to illustrate the treatment of higher order equations, let us consider the 1-dimentional wave equation $u_{t t}=u_{s s}, s \in \mathbb{R}$, with the initial condition $u(0, s)=u_{0}(s)$ and $u_{t}(0, s)=u_{1}(s)$. As is well-known, the theory of $\left(C_{0}\right)$-semigroup can be applied to this equation, by introducing an appropriate function space (see Yosida [27]). Here, we consider the problem over the space $L_{2}=L_{2}(R) \times L_{2}(R)$. Putting $u=v_{1}$ and $u_{t}=v_{2}$, we can write the equation as

$$
\begin{aligned}
& (\partial / \partial t) v_{1}=v_{2}, \quad(\partial / \partial t) v_{2}=(\partial / \partial s)^{2} v_{1}, \\
& v_{1}(0, s)=u_{0}(s), \quad v_{2}(0, s)=u_{1}(s), \quad s \in \mathbf{R} .
\end{aligned}
$$

Applying the Fourier transform, the problem is reduced to the following:

$$
(d / d t) \hat{v}(t, \xi)=P(\xi) \hat{v}(t, \xi), \quad \hat{v}(0, \xi)=\left(\begin{array}{l}
\hat{u}_{0}(\xi) \\
\hat{u}_{1}(\xi)
\end{array}\right)
$$

where

$$
P(\xi)=\left(\begin{array}{cc}
0 & 1 \\
-\xi^{2} & 0
\end{array}\right)
$$

and $\hat{v}$ denotes the Fourier transform of $v$. For each $\lambda>0$ we have that $(\lambda E-P(\xi))^{-1}=\left(\lambda^{2}+\xi^{2}\right)^{-1}[\lambda E+P(\xi)]$ for $\xi \in \mathbf{R}$, and so, the operator $P$ has the resolvent set containing $\{\lambda>0\}$. The solution of (4.21) is obtained by

$$
e^{t P(\xi)} \hat{v}(\xi)=\left(\begin{array}{cc}
\cos \xi t & \xi^{-1} \sin \xi t \\
-\xi \sin \xi t & \cos \xi t
\end{array}\right) \hat{v}(\xi)=\cos \xi t E \hat{v}(\xi)+t \frac{\sin \xi t}{\xi t} P(\xi) \hat{v}(\xi) .
$$

By the Fourier-Plancherel theorem we have that $\left\|U_{t} v\right\|=\left\|e^{t P} \hat{v}\right\| \leqq\{\|\hat{v}\|$ $+t\|P \hat{v}\|\}=\|v\|+t\|A v\| \leqq(1+t)\|v\|_{1}$ for $v \in D(A)$. Also, by using the relation (4.18), we see that $\left\|\lambda^{n}(\lambda-A)^{-n} v\right\| \leqq(1+n / \lambda)\|v\|_{1}$ for $\lambda>0$ and n. This implies that $A \in \mathbb{S}_{2}(1,1)$. This means that for each $v \in D\left(A^{2}\right)$ $(A=P(D))$, the inverse Fourier transform of $e^{t P(\xi)} v(\xi)$ gives a solution $U_{t} v$ of the $A C P_{1}$ for $A$ associated with the initial value $v$. Finally, we note that the equation (4.21) is correctly posed in the sense of Petrowsky, since the eigenvalues of $P(\xi)$ are $\pm i \xi$.

\section{Distribution Semigroups}

In this section we discuss some relationships among the notion of 
distribution semigroup and the results obtained in the preceding sections.

In Section 4 we treated classes $\mathcal{S S}_{1}(\omega, k, T), i=1,2$, and 3 . We first consider other classes $\mathscr{S}_{1}(\omega, \infty)$ and $\mathscr{S}_{2}(\omega, \infty)$ and discuss the construction of the semigroup solution of $A C P$ in a Fréchet space.

Let $A \in \mathfrak{C}(X)$ and $\omega \in \mathbf{R}$ and then let us consider condition $(I ; \omega)$ and the following condition:

$(I I ; \infty)$ let $Y=\bigcap_{n \geqq 1} D\left(A^{n}\right)$; then for every $T>0$ there exist a $k=$ $k(T) \in \mathbf{Z}_{+}$and $\left.M=M \stackrel{n \geq 1}{T}\right)>0$ such that

$$
\left\|\xi^{n} R(\xi ; A)^{n} y\right\| \leqq M\|y\|_{k} \quad \text { for } y \in Y, \xi>\omega \text { and } n / \xi \in[0, T] \text {. }
$$

We denote by $\mathbb{S}_{1}(\omega, \infty)$ the class of all closed operators in $X$ satisfying $(I ; \omega)$ and $(I I ; \infty)$. We also consider another class of operators $A$ in $X$ satisfying $(I ; \omega)$ and

$\left(I I_{\exp } ; \infty\right)$ let $Y=\bigcap_{n \geqq 1} D\left(A^{n}\right)$; then there exist a $k \in \mathbf{Z}_{+}$and numbers $M>0$ and $\omega_{1} \geqq \omega$ such that

$$
\left\|R(\xi ; A)^{n} y\right\| \leqq\left(\xi-\omega_{1}\right)^{-1} M\|y\|_{k} \quad \text { for } y \in Y, n \in \mathbf{Z}_{+} \text {and } \xi>\omega_{1}
$$

instead of $(I I ; \infty)$. We denote such a class by $\dot{B S}_{2}(\omega, \infty)$.

Remark 5.1. Condition $(I I ; \infty)$ states that $A \in \mathcal{S}_{1}(\omega, \infty, T)$ for every $T>0$. Also, we observed in Section 3 that $Y$ become a Fréchet space. Hence, in view of the principle of uniform boundedness, we see that $\left(I I_{\exp } ; \infty\right)$ is equivalent to

$\left(I I_{\text {exp }} ; \infty\right)^{\prime}$ there is an $\omega_{1} \geqq \omega$ such that $\sup \left\{\left\|\xi^{n} R\left(\xi ; A-\omega_{1}\right)^{n} y\right\|\right.$; $\left.\xi>\omega_{1}, n \in \mathbf{Z}_{+}\right\}<+\infty$.

Therefore, $\mathscr{S}_{1}(\omega, \infty) \supset \mathscr{S}_{1}(\omega, k)$ and $\mathscr{S}_{2}(\omega, \infty) \supset \mathscr{S}_{2}(\omega, k)$ for all $k \in \mathbb{Z}_{+}$.

First, as a direct consequence of Theorem 3.3, we obtain the following:

Theorem 5.2. Let $A \in \mathscr{B}_{1}(\omega, \infty)$, then $A \mid Y$ is the infinitesimal generator of a locally equicontinuous semigroup $\left\{T_{t} ; t \geqq 0\right\}$ on $Y$ such that $T_{t} y$ is infinitely differentiable in $\overline{\mathbf{R}}_{+}$for $y \in Y$.

Also, by employing the theory of equicontinuous semigroups in a sequentially complete, locally convex space, we obtain

Theorem 5.3. Let $A \in \mathfrak{}(X)$ satisfy condition $(I ; \omega)$ and let $Y$ 
$=\bigcap_{n \geqq 1} D\left(A^{n}\right)$. Then $A \mid Y-\omega_{1}$ is the infinitesimal generator of an equicontinuous semigroup $\left\{e^{-\omega_{1} t} T_{t} ; t \geqq 0\right\}$ on $Y$ for some $\omega_{1} \geqq \omega$, if and only if $A$ satisfies $\left(I I_{\mathrm{exp}} ; \infty\right)$.

Proof. Assume that $A \in \mathbb{B S}_{2}(\omega, \infty)$, then Theorem 5.2 yields that $A \mid Y$ is the infinitesimal generator of a semigroup $\left\{T_{t} ; t \geqq 0\right\}$ in the Fréchet space $Y$. Also, by Theorem 3.3 (a), there exist a $k \in \mathbb{Z}_{+}$and an $M>0$ such that $\left\|T_{t} y\right\| \leqq M e^{\omega_{1} t}\|y\|_{k}$ for $y \in Y$. This means that $\left\{e^{-\omega_{1} t} T_{t} ; t \geqq 0\right\}$ is equicontinuous on $Y$. Hence, $A \mid Y-\omega_{1}$ is the infinitesimal generator of the equicontinuous semigroup $\left\{e^{-\omega_{1} t} T_{t} ; t \geqq 0\right\}$ on $Y$. Conversely, if $A \mid Y-\omega_{1}$ is the infinitesimal generator of an equicontinuous semigroup for some $\omega_{1}$, then $\left\{\xi^{n} R\left(\xi ; A-\omega_{1}\right)^{n} \mid Y ; \xi>0, n \in \mathbf{Z}_{+}\right\}$is equicontinuous on $Y$. Hence, there exist a $k \in \mathbf{Z}_{+}$and an $M>0$ such that $\left\|\xi^{n} R\left(\xi+\omega_{1} ; A\right)^{n} y\right\| \leqq M\left\|_{1} y\right\|_{k}$ for $\xi>0$ and $y \in Y$, that is, $\left\|\left(\xi-\omega_{1}\right)^{n} R(\xi ; A)^{n} y\right\| \leqq M\|y\|_{k}$ for $\xi>\omega_{1}$ and $y \in Y$. Therefore, $A \in \mathbb{S}_{2}(\omega, \infty)$.

Q.E.D.

Next, we consider the case in which $\bar{Y}=X$. In such a case we can apply important results on the characterizations of regular and exponential distribution semigroups which were given by Chazarain [1], Fujiwara [7], Lions [12] and Ushijima [24] and we can consider some relationships among the notion of distribution semigroup and the classes of operators, SS $_{i}(\omega, k), i=1,2,3 ; k=0,1, \ldots, \infty$.

Theorem 5.4. Let $\omega \in \mathbf{R}, A$ a densely defined closed operator in $X$, and $Y=\bigcap_{n \geqq 1} D\left(A^{n}\right)$. Then $A$ is the infinitesimal generator of an R.D.S.G. $T$ on $X$ if it satisfies any one of the following:

(i) $A \in \mathcal{S S}_{1}(\omega, \infty)$ and $\bar{Y}=X$,

(ii) for every $T>0$ there exists a $k(T) \in \mathbf{Z}_{+}$such that $A \in \mathbb{S}_{1}(\omega, k$ $(T), T)$. Furthermore, in this case, $T(\phi) y=\int_{0}^{\infty} \phi(t) T_{t} y d t$ for $y \in Y$ and $\phi \in D\left(\mathbf{R}_{+}\right)$, where $\left\{T_{t} ; t \geqq 0\right\}$ is the locally equicontinuous semigroup constructed in $Y$ by Theorem 5.2 .

Proof. Assume condition (i), then by Theorem 5.2, $A \mid Y$ is the in finitesimal generator of a locally equicontinuous semigroup $\left\{T_{t} ; t \geqq 0\right\}$ on the Fréchet space $Y$. Since $\bar{Y}=X$, Lemma 3.6 yields that $\overline{A \mid Y}=A$. Therefore, by using Ushijima [24; Theorem 2] we see that $A$ is the infinitesimal generator of an R,D.S.G. $T$ such that 


$$
T(\phi) y=\int_{0}^{\infty} \phi(t) T_{t} y d t
$$

for $y \in Y$ and $\phi \in D\left(\mathbf{R}_{+}\right)$. Next, suppose that (ii) holds. Then, $A \in \mathbb{S}_{1}(\omega, \infty)$ by definition and Theorem 3.9 yields that $\bar{Y}=X$. Hence, (i) holds.

Q.E.D.

Theorem 5.5. An operator $A$ in $X$ is the infinitesimal generator of an E.D.S.G. T, if and only if $A$ is a densely defined, closed operator satisfying either of the following conditions:

(i) $A \in \mathcal{B S}_{2}(\omega, k)$ for some $\omega \in \mathbf{R}$ and $k \in \mathbf{R}_{+}$,

(ii) $A \in \mathbb{S}_{2}(\omega, \infty)$ for some $\omega \in \mathbf{R}$ and also $\bar{Y}=X$,

(iii) $\rho(A) \neq \emptyset, \bar{Y}=X$, and for some $\omega_{1} \in \mathbb{R}, A \mid Y-\omega_{1}$ is the infinitesimal generator of an equicontinuous semigroup $\left\{T_{t} ; t \geqq 0\right\}$ on $Y$ which is a Fréchet space in the sense of Lemma 3.1.

(iv) $A \in \mathbb{B}_{3}(\gamma, l)$ for some $\gamma \in \mathbf{R}$ and $l \in \mathbf{Z}_{+}$.

Proof. Let $A$ be a densely defined, closed operator satisfying (i). Then $A \in \mathbb{S}_{2}(\omega, \infty)$ by definition and Theorem 3.9 states that $\bar{Y}=X$; and hence (ii) is satisfied. Next, suppose (ii). Then by Theorem 5.3, $A \mid Y-\omega_{1}$ is the infinitesimal generator of an equicontinuous semigroup $\left\{T_{t} ; t \geqq 0\right\}$ on the Fréchet space $Y$. Since $\bar{Y}=X$, Lemma 3.6 yields that $A=\overline{A \mid Y}$. Hence, (iii) holds. Now, let us assume that (iii) holds. Then by Fujiwara [7; Theorem 3], $A$ is the infinitesimal generator of an E.D.S.G. Let $A$ be the infinitesimal generator of an E.D.S.G. $T$. Then by Lions $[12$; Theorem 6.1], we see that $A \in \mathbb{S}_{3}(\gamma, l)$ for some $\gamma \in \mathbf{R}$ and $l \in \mathbf{Z}_{+}$. Thus, (iv) is satisfied. Finally, let (iv) be satisfied. Then from Theorem 4.7 (a) it follows that $A \in \mathbb{S}_{2}(\omega, l+2)$ for an $\omega>\max \{0, r\}$, which means that (i) holds.

Q.E.D.

Remark 5.6. Theorem 5.5 is a combination of Lions' result and Fujiwara's one. Combining the results obtained in Section 4, we see that Theorem 5.5 gives some informations on the continuity of E.D.S.G. at the origin $t=0$ and the regularity in the sense of the norm topology of $X$.

Now, in the remainder of this section, we discuss the semigroup of bounded operators which can be extended to an R.D.S.G. By treating such semigroups, the Feller-type condition is naturally introduced. 
Definition 5.7. A semigroup $\left\{T_{t} ; t>0\right\}$ of bounded operators is said to be extended to an R.D.S.G. if there exists an R.D.S.G. $T$ such that for any $x \in X$ and $\phi \in D\left(\mathbf{R}_{+}\right)$,

$$
T(\phi) x=\int_{0}^{\infty} \phi(t) T_{t} x d t .
$$

Recently, Ushijima [25; Theorem 1] characterized such a semigroup $\left\{T_{t}\right\}$ in terms of strong infinitesimal generator $A_{s}$ which is defined through the relations:

$D\left(A_{s}\right)=\left\{x \in X ; \exists \lim _{\eta \rightarrow+0} \eta^{-1}\left[T_{\eta}-I\right] x=y \in \Sigma\right\}$ and $A_{s} x=y$ for $x \in D\left(A_{s}\right)$.

Theorem 5.8. (Ushijima) Let $\left\{T_{t} ; t>0\right\}$ be a semigroup of bounded operators and $A_{s}$ be its strong infinitesimal generator. Then $\left\{T_{t}\right\}$ can be extended to an R.D.S.G. $T$ if and only if $\overline{D\left(A_{s}\right)}=X, A_{s}$ is closable, $\rho(A)$ $\neq \varnothing$, and there exists a $k \in \mathbf{Z}_{+}$such that $D\left(A^{k}\right) \subset \Sigma$, where $\Sigma$ is the continuity set and $A=\bar{A}_{s}$. Furthermore, in this case, the extension $T$ is unique, $A$ is its infinitesimal generator and $A$ satisfies $(I ; \omega)$.

We then study some basic properties of the semigroup which can be extended to an R.D.S.G. Throughout the remainder of this section, let $\left\{T_{t}\right\}$ be a semigroup of bounded operators with the type $\omega_{0}, A_{0}$ (resp. $A_{s}$ ) be the (resp. strong) infinitesimal generator, and $\Sigma$ be the continuity set.

Lemma 5.9. Assume that there is an $A \in \mathfrak{S}(X)$ with non-empty resolvent set and that $D\left(A^{k}\right) \subset \Sigma$ for some $k \in \mathbf{Z}_{+}$. Let $\omega_{1}>\omega_{0}$, then there is a number $M>0$ such that

$$
\left\|T_{t}\right\|_{k} \leqq M e^{\omega_{1} t}, \quad t>0
$$

Proof. We observe that $T_{t} \in \mathfrak{B}\left(D\left(A^{k}\right), X\right)$ for $t>0$. Let $\omega_{1}>\omega_{0}$. Then the resonance theorem yields that $\sup _{t>0}\left\|e^{-\omega_{1} t} T_{t}\right\|_{k}=M<+\infty$, from which (5.2) follows.

Q.E.D.

For $\lambda$ with $\operatorname{Re}(\lambda)>\omega_{1}>\omega_{0}$, we define an operator $R_{0}(\lambda)$ by the relation

$$
R_{0}(\lambda) x=\int_{0}^{\infty} e^{-\lambda t} T_{t} x d t
$$

whenever the right integral makes sense. We note that $D\left(R_{0}(\lambda)\right) \supset \Sigma$ for 
$\operatorname{Re}(\lambda)>\omega_{1}$.

The following is important for our arguments in the sequel; the central part of the proof is based on that of the characterization of $(A)$ semigroups, refer to Hille-Phillips [8; Theorem 12.5.1].

Theorem 5.10. Let $A \in \mathbb{E}(X)$ satisfy $(I ; \omega)$ and $D\left(A^{k}\right) \subset \Sigma$ for some $\omega \in \mathbf{R}$ and $k \in \mathbf{Z}_{+}$. If $R(\xi ; A)\left|D\left(A^{k}\right)=R_{0}(\xi)\right| D\left(A^{k}\right)$ for $\xi>\omega_{1}>\max \left\{\omega, \omega_{0}\right\}$, then $A \in \mathbb{F}_{2}(\omega, k)$ and the following condition of Feller type is satisfied: $(F ; k)$ for every $\varepsilon>0$, there is a positive number $M_{\varepsilon}$ and for every $x \in D$ $\left(A^{k}\right)$, there is a positive number $\xi_{0} \equiv \xi_{0}(\varepsilon, x)\left(>\omega_{1}\right)$ such that

$$
\left\|\xi^{n} R(\xi ; A)^{n} x\right\| \leqq M_{\varepsilon}\|x\| \quad \text { for } \xi>\xi_{0} \text { and } \varepsilon \leqq n / \xi \leqq 1 / \varepsilon \text {. }
$$

Proof. First, we show that $A$ satisfies $(F ; k)$. Let $\hat{T}_{t} \equiv e^{-\omega_{1} t} T_{t}, t>0$, and $\hat{A}=A-\omega_{1}$, then $R(\xi ; A)=R\left(\xi-\omega_{1} ; \hat{A}\right)$ for $\xi>\omega_{1}$ and $e^{-\xi t} T_{t}=$ $e^{-\left(\xi-\omega_{1}\right) t} \hat{T}_{t}$ for $t>0$. Hence, we have

$$
R(\xi ; \hat{A}) x=\int_{0}^{\infty} e^{-\xi t} \hat{T}_{t} x d t \quad \text { for } \xi>0 \text { and } x \in D\left(A^{k}\right) .
$$

Using the resolvent equation we have

$$
R(\xi ; \hat{A})^{n} x=(n-1) !^{-1} \int_{0}^{\infty} e^{-\xi t} t^{n-1} \hat{T}_{t} x d t,
$$

for $\xi>0, x \in D\left(A^{k}\right)$ and $n \in \mathbf{Z}_{+}$. Also, since $\left\{\hat{T}_{t}\right\}$ is of negative type, $\phi(t)$ $\equiv \sup _{\sigma>t}\left\|e^{-\omega_{1} \sigma} T_{\sigma}\right\|$ is finite at each $t>0$, nonnegative, nonincreasing and of negative type. Let $0<\delta^{\prime}<\delta$ and $q=\delta^{\prime} / \delta(<1)$ and let us decompose the right side of (5.4) into two parts;

$$
J_{1}=(n-1) !^{-1} \int_{0}^{\delta^{\prime}}, \quad J_{2}=(n-1) !^{-1} \int_{\delta^{\prime}}^{\infty},
$$

then for $x \in D\left(A^{k}\right),\left\|J_{1}\right\| \leqq(n-1) !^{-1} M\|x\|_{k} \xi^{-n} \int_{0}^{\delta^{\prime} \xi} e^{-s} s^{n-1} d s$ and $\left\|J_{2}\right\| \leqq$ $\xi^{-n} \psi\left(\delta^{\prime}\right)\|x\|$. If $\xi>0$ and $n \geqq \xi \delta$, then by the same argument as in [8; (12.5.6), p. 374] we have that

$$
(n-1) !^{-1} \int_{0}^{\delta^{\prime} \xi} e^{-s} s^{n-1} d s \leqq q / n(1-q)^{2},
$$


Consequently, we have that $\left\|\xi^{n} R(\xi ; \hat{A})^{n} x\right\| \leqq \psi\left(\delta^{\prime}\right)\|x\|_{\|}+M\left\|_{i} x\right\|_{k} q / n(1-q)^{2}$ for $\xi>0$ and $n \geqq \xi \delta$. This means that for $x \in D\left(A^{k}\right), \delta^{\prime}$ and $\delta$ with $0<$ $\delta^{\prime}<\delta$, and $\eta>0$, there exists a $\mu_{0} \equiv \mu_{0}\left(x, \eta, \delta^{\prime}, \delta\right)$ such that

$$
\left\|\xi^{n} R(\xi ; \hat{A})^{n} x\right\| \leqq\left\{\psi\left(\delta^{\prime}\right)+\eta\right\}\|x\|
$$

for $\xi>\mu_{0}$ and $n \geqq \xi \delta$. In fact, to obtain the estimate $M\|x\|_{k} q / n(1-q)^{2} \leqq$ $\eta\|x\|$, we must take $n \geqq M\|x\|_{k} q / \eta \cdot\|x\|(1-q)^{2}$. But, it is possible if $\xi \geqq M\|x\|_{k} q / \eta \delta\|x\|(1-q)^{2}$; this guarantees the existence of such a number $\mu_{0}$. Now, for $x \in D\left(A^{k}\right)$ and $\varepsilon>0$, we take $\delta^{\prime}, \delta$ with $0<\delta^{\prime}<\delta<\varepsilon$ and $\lambda_{0}$ $\equiv \mu_{0}\left(x, \eta, \delta^{\prime} \delta\right)+\omega_{1}$, then we can find a positive number $M_{\varepsilon}$ such that $\sup \left\{\left(1+\left(\xi-\omega_{1}\right)^{-1} \omega_{1}\right)^{n}\left\{\psi\left(\delta^{\prime}\right)+\eta\right\} ; \xi>\lambda_{0}, \varepsilon \leqq n / \xi \leqq 1 / \varepsilon\right\} \leqq M_{\varepsilon}$.

Therefore, $\left\|\xi^{n} R(\xi ; A)^{n} x\right\| \leqq M_{\varepsilon}\|x\|$ for $\xi>\lambda_{0}$ and $\varepsilon \leqq n / \xi \leqq 1 / \varepsilon$; this is condition $(F ; k)$. Next, we show that $\left(I I_{\exp } ; k\right)$ holds for $\omega_{1}$ and the number $M$ implied by Lemma 5.9. By (5.2) and (5.4), we have

$$
\left\|R(\xi ; \hat{A})^{n} x\right\| \leqq(n-1) !^{-1} M\|x\|_{k} \xi^{-n} \int_{0}^{\infty} e^{-s} s^{n-1} d s \leqq \xi^{-n} M\|x\|_{k} .
$$

Hence, by replacing $\xi$ by $\xi-\omega_{1}$ in the above estimate, we obtain

$$
\left\|R(\xi ; A)^{n} x\right\| \leqq\left(\xi-\omega_{1}\right)^{-n} M\|x\|_{k} . \quad \text { Q.E.D. }
$$

Lemma 5.11. Let $\omega_{1}>\omega_{0}$ and $R_{0}(\lambda)$ be defined for $\operatorname{Re}(\lambda)>\omega_{1}$ by (5.3). Then for every $x \in \Sigma$ and $\operatorname{Re}(\lambda)>\omega_{1}, R_{0}(\lambda) x \in D\left(A_{s}\right)$ and $\left(\lambda-A_{s}\right)$ $R_{0}(\lambda) x=x$.

Proof. Let $A_{\eta}=\eta^{-1}\left[T_{\eta}-I\right]$ for $\eta>0$, then $A_{\eta} \in \mathfrak{B}(X)$. Let $x \in \Sigma$ and $\operatorname{Re}(\lambda)>\omega_{1}$, then we have

$$
\left\{\begin{aligned}
A_{\eta} R_{0}(\lambda) x & =\eta^{-1} \int_{0}^{\infty} e^{-\lambda t}\left[T_{t+\eta} x-T_{t} x\right] d t \\
& =\eta^{-1}\left(e^{\lambda \eta}-1\right) \int_{0}^{\infty} e^{-\lambda t} T_{t} x d t-\eta^{-1} \int_{0}^{\eta} e^{\lambda(\eta-t)} T_{t} x d t \\
& \rightarrow \lambda R_{0}(\lambda) x-x, \quad \text { as } \eta \rightarrow+0 .
\end{aligned}\right.
$$

This means that $R_{0}(\lambda) x \in D\left(A_{0}\right) \subset \Sigma$ and $\left(\lambda-A_{0}\right) R_{0}(\lambda) x=x$, where $A_{0}$ is the infinitesimal generator of $\left\{T_{t}\right\}$. Since $\lim _{\delta \rightarrow+0} T_{\delta} R_{0}(\lambda) x=R_{0}(\lambda) x$, we have that $T_{\delta} A_{0} R_{0}(\lambda) x=T_{\delta}\left(\lambda R_{0}(\lambda) x-x\right) \stackrel{\delta \rightarrow+0}{\rightarrow \lambda R_{0}}(\lambda) x-x=A_{0} R_{0}(\lambda) x$. 
Therefore, $\quad R_{0}(\lambda) x \in D\left(A_{s}\right)$ and $A_{0} R_{0}(\lambda) x=A_{s} R_{0}(\lambda) x$. Consequently, $\left(\lambda-A_{s}\right) R_{0}(\lambda) x=x$.

In view of the results mentioned above, we see that a semigroup which can be extended to an R.D.S.G. has the following property.

Theorem 5.12. Assume that $\left\{T_{t} ; t>0\right\}$ can be extended to an R.D.S.G. T. Let $A=\bar{A}_{s}$. Then $A \in \mathcal{B S}_{2}(\omega, k)$ and condition $(F ; k)$ holds for some $\omega \in \mathbb{R}$ and $k \in \mathbb{Z}_{+}$.

Proof. Since $A$ is the infinitesimal generator of an R.D.S.G. $T$ by Theorem 5.8, $A$ satisfies $(I ; \omega)$ for some $\omega \in \mathbb{R}$. Let $\omega_{1}>\max \left\{\omega, \omega_{0}\right\}$, then by Lemma 5.9 there is an $M>0$ such that (5.2) holds. Also, Lemma 5.11 states that $x=\left(\lambda-A_{s}\right) R_{0}(\lambda) x=(\lambda-A) R_{0}(\lambda) x$ for $x \in \Sigma$ and $\operatorname{Re}(\lambda)$ $>\omega_{1}$. Hence, by applying the resolvent of $A$ at $\xi>\omega_{1}$ on both sides of this equality for $\lambda=\xi$, we see that $R_{0}(\xi) x=R(\xi ; A) x$ for $\xi>\omega_{1}$ and $x \in \Sigma$. On the other hand, it follows from Theorem 5.8 that $D\left(A^{k}\right) \subset \Sigma$ for some $k \in \mathbb{Z}_{+}$. Therefore, Theorem 5.10 implies that $A$ satisfies $\left(I I_{\text {exp }} ; k\right)$ and $(F ; k)$.

Q.E.D.

Remark 5.13. By virtue of Theorem 5.5, we see that the semigroup $\left\{T_{t}\right\}$ of bounded operators which can be extended to an R.D.S.G. is necessarily extended to an E.D.S.G.

Remark 5.14. In the next section we shall introduce certain classes $\left(C_{(k)}\right), k=0,1,2, \ldots, \infty$, of semigroups of bounded operators. The semigroup of class $\left(C_{(k)}\right)$ has the property that $D\left(\bar{A}_{0}^{k}\right) \subset \Sigma$, where $A_{0}$ denotes the infinitesimal generator and $\Sigma$ denotes the continuity set. It will then be shown that a densely defined, closed operator $A$ in $X$ satisfying $(I ; \omega)$, $\left(I I_{\exp } ; k\right)$ and $(F ; k)$ for some $\omega \in \mathbb{R}$ and $k \in \mathbb{Z}_{+}$is the closure of the infinitesimal generator $A_{0}$ of a unique semigroup $\left\{T_{t} ; t>0\right\}$ of class $\left(C_{(k)}\right)$. Therefore, if a semigroup $\left\{\widetilde{T}_{t} ; t>0\right\}$ can be extended to an R.D.S.G. $T$, then $A=\bar{A}_{s}$ is the closure of the infinitesimal generator $A_{0}$ of a semigroup $\left\{T_{t}\right\}$ (of some class $\left(C_{(k)}\right)$ ). But, $\widetilde{T}_{t} \equiv T_{t}$ by the unicity of the semigroup; and hence it follows that $A=\bar{A}_{s}=\bar{A}_{0}$. Conversely, as will be shown in the next section, the infinitesimal generator $A_{0}$ of a semigroup $\left\{T_{t}\right\}$ of class $\left(C_{(k)}\right)$ is closable and $A=\bar{A}_{0}$ satisfies $(I ; \omega),(I I ; k),(F ; k)$ and $\bar{Y}=X$. Since $A \mid Y \subset A_{s} \subset A_{0} \subset A$ by Theorem 4.6 and since $Y$ is a core of 
$A$ by Lemma 3.6 , we see that $\overline{A \mid Y}=\bar{A}_{s}=\bar{A}_{0}=A$. Hence, $A_{s}$ in Theorem 5.8 can be replaced by $A_{0}$ as follows:

Theorem 5.8'. A semigroup $\left\{T_{t}\right\}$ of bounded operators can be extended to an R.D.S.G. $T$ if and only if the infinitesimal generator $A_{0}$ is densely defined, closable and $A=\bar{A}_{0}$ satisfies that $\rho(A) \neq \varnothing$ and $D\left(A^{k}\right) \subset \Sigma$ for some $k \in \mathbf{Z}_{+}$.

According to Ushijima [25], a semigroup of bounded operators $\left\{T_{t}\right\}$ is called a strongly continuous semigroup (C.D.S.G.) if it admits a regular distributional extension $T$. In view of Remark 5.14 mentioned above, we obtain the following type of characterization of C.D.S.G.:

Theorem 5.15. An operator $A$ is the closure of the infinitesimal generator of a C.D.S.G. if and only if $A \in \mathbb{S}_{2}(\omega, k)$ and $(F ; k)$ is satisfied for some $\omega \in \mathbf{R}$ and $k \in \mathbb{Z}_{+}$.

The above theorem states that every C.D.S.G. belongs to some class $\left(C_{(k)}\right)$ and conversely. Hence, the basic structure and the regularity of a C.D.S.G. will be seen by considering the characterization of $\left(C_{(k)}\right)$-semigroups.

Finally, we consider condition of Feller type $(F ; k)$. Let $A$ be a densely defined, closed operator in $X$ belonging to $\mathcal{S}_{1}(\omega, k)$ and $\left\{U_{t} ; t \geqq 0\right\}$ $\mathcal{C B}\left(D\left(A^{k}\right), X\right)$ be the one-parameter family obtained by Theorem 4.2. Then for $k^{\prime} \in \mathbf{Z}_{+}$with $k^{\prime} \geqq k$ we can consider the following condition:

$\left(C ; k^{\prime}\right)$ for every $t>0$ there exists a number $M_{t}>0$ such that

$$
\left\|U_{t} x\right\| \leqq M_{t}\|x\| \quad \text { for } x \in D\left(A^{k^{\prime}}\right) .
$$

This condition is called the condition of correct posedness of $A C P$ for $A \in \mathbb{S}_{1}(\omega, k)$. We discuss some relations between conditions $(F ; k)$ and $(C ; k)$.

Lemma 5.16. Let $A \in \mathfrak{S}(X)$ and $\overline{D(A)}=X$. Let $A \in \mathbb{S}_{1}(\omega, k)$ and $\left\{U_{t} ; t \geqq 0\right\} \subset \mathfrak{B}\left(D\left(A^{k}\right), X\right)$ be the corresponding one-parameter family obtained by Theorem 4.2. If condition $\left(C ; k^{\prime}\right)$ is satisfied for some $k^{\prime} \geqq k$, then there exists a semigroup of bounded operators $\left\{T_{t} ; t>0\right\}$ such that $T_{t} \mid D$ $\left(A^{k}\right)=U_{t}$ for $t>0$. Furthermore, $D\left(A^{k}\right) \subset \Sigma$.

Proof. Since $\overline{D\left(A^{k^{\prime}}\right)}=X$ by Lemma 2.7 , each of $U_{t} \mid D\left(A^{k^{\prime}}\right), t>0$, 
admits a unique continuous extension $T_{t} \in \mathfrak{B}(X)$. Theorem 4.2 (d) and Lemma 2.7 implies that $\left\{T_{t}\right\}$ has the semigroup property. Since $T_{t} x$ is strongly measurable with respect to $t$ in $\overline{\mathbb{R}}_{+}$for $x \in X,\left\|T_{t}\right\|$ is measurable. Also, $\left\|T_{t}\right\|$ is a submultiplicative function of $t$. Therefore, for every $\varepsilon>0$ there is a number $M_{\varepsilon}>0$ such that $\sup \left\{\left\|T_{t}\right\| ; \varepsilon \leqq t \leqq 1 / \varepsilon\right\} \leqq M_{\varepsilon}$, see Hille-Phillips $\left[8 ;\right.$ p. 242]. Hence, $\left\{T_{t}\right\}$ forms a semigroup of bounded operators. By the way, $D\left(A^{k^{\prime}}\right)$ is dense in $\left[D\left(A^{k}\right)\right]$ by Lemma 2.7; and hence it follows that $T_{t} \mid D\left(A^{k}\right)=U_{t}$ for $t>0$. Since $U_{t} x$ was strongly continuous in $t \geqq 0$ for $x \in D\left(A^{k}\right)$, we see that $D\left(A^{k}\right) \subset \Sigma$. $\quad$ Q.E.D.

Theorem 5.17. Let $A \in \mathbb{S}(X)$ and $\overline{D(A)}=X$. Then the following conditions are equivalent:

(i) $A \in \mathbb{S}_{1}(\omega, k)$ and $\left(F ; k^{\prime}\right)$ holds for some $k^{\prime} \geqq k$,

(ii) $A \in \mathcal{B S}_{2}(\omega, k)$ and $(F ; k)$ holds,

(iii) $A \in \mathbb{B S}_{2}(\omega, k)$ and $(C ; k)$ holds,

(iv) $A \in \mathbb{S}_{1}(\omega, k)$ and $\left(C ; k^{\prime}\right)$ holds for some $k^{\prime} \geqq k$.

Proof. Assume that (i) is satisfied for $k^{\prime}>k$. Then (a) of Theorem 4.2 implies that $\sup \left\{\left\|U_{t} x\right\| ; t \in[\varepsilon, 1 / \varepsilon]\right\} \leqq M_{\varepsilon}\|x\|$ for $\varepsilon>0$ and $x \in D\left(A^{k^{\prime}}\right)$. Hence, by Lemma 5.16 we obtain a semigroup of bounded operators $\left\{T_{t}\right.$; $t>0\}$ such that $T_{t} \mid D\left(A^{k}\right)=U_{t}$ for $t>0$. Let $\omega_{0}$ be the type and $\omega_{1}>$ $\max \left\{\omega, \omega_{0}\right\}$. Then, by Lemma 5.9 , a sufficiently large $M>0$ can be found in such a way that $\left\|U_{t} x\right\| \leqq M e^{\omega_{1} t}\|x\|_{k}$ for $t \geqq 0$ and $x \in D\left(A^{k}\right)$. Hence, by the same way as in the proof of Theorem 4.3 (f), we see that $R(\xi ; A) x$ $=R_{0}(\xi) x=\int_{0}^{\infty} e^{-\xi t} T_{t} x d t$ for $\xi>\omega_{1}$ and $x \in D\left(A^{k}\right)$. Therefore, Theorem 5.10 yields that (ii) holds. Assume that (ii) is satisfied. Then Theorem 4.2 (a) implies that $\sup \left\{\left\|U_{t} x\right\| ; \varepsilon \leqq t \leqq 1 / \varepsilon\right\} \leqq M_{\varepsilon}\|x\|$ for $\varepsilon>0$ and $x \in D$ $\left(A^{k}\right)$. This is nothing but condition $(C ; k)$. Hence, (iii) follows. (iii) $\Rightarrow$ (iv) is evident. Finally, suppose (iv). Then Lemma 5.16 states that there is a semigroup $\left\{T_{t}\right\}$ such that $T_{t} \mid D\left(A^{k}\right)=U_{t}$ for $t>0$. Hence, by the same way as in the proof of the implication (i) $\Rightarrow$ (ii) mentioned above, we see that (ii) holds.

Q.E.D.

\section{Class $\left(\boldsymbol{C}_{(\boldsymbol{k})}\right)$}

In this section we introduce some classes of semigroups of bounded 
operators and discuss their characterizations in terms of resolvents of the infinitesimal generators.

Let $\left\{T_{t} ; t>0\right\}$ be a semigroup of bounded operators, $X_{0} \equiv \bigcup_{t>0} T_{t}[X]$, $\omega_{0}$ be the type, and $\Sigma=\left\{x \in X ; \lim _{t \rightarrow+0} T_{t} x=x\right\}$ be the continuity set. We define $R_{0}(\lambda)$, the Laplace transform of $T_{t}$, by (5.3) for $\lambda \in \mathbb{C}$ and $x \in X$. We noted that $D\left(R_{0}(\lambda)\right) \supset \Sigma$ provided that $\operatorname{Re}(\lambda)>\omega_{0}$. Also, let us denote by $A_{0}$ the infinitesimal generator and let $Y_{0} \equiv \bigcap_{n \geqq 1} D\left(A_{0}^{n}\right.$.)

Now, we consider a semigroup $\left\{T_{t} ; t>0\right\}$ with the properties

(C.1) $\bar{X}_{0}=X$,

(C.2) there is an $\omega_{1}>\omega_{0}$ such that for $\lambda$ with $\operatorname{Re}(\lambda)>\omega_{1}$ there is an $R(\lambda) \in \mathfrak{B}(X)$ and $R(\lambda)\left|X_{0}=R_{0}(\lambda)\right| X_{0}$,

(C.3) if $R(\lambda) x=0$ for $\lambda>\omega_{1}$, then $x=0$.

Remark 6.1. Since $\lim _{\eta \rightarrow+0} T_{\eta} R_{0}(\lambda) x=\lim _{\eta \rightarrow+0} R_{0}(\lambda) T_{\eta} x=R_{0}(\lambda) x$ for $x \in \Sigma$ and $\operatorname{Re}(\lambda)>\omega_{1}$, it follows that $R(\lambda)\left|\Sigma=R_{0}(\lambda)\right| \Sigma$ for $\operatorname{Re}(\lambda)>\omega_{1}$.

In the sequel we discuss some properties of this kind of semigroup.

Lemma 6.2. Let $A_{0}$ be the infinitesimal generator of a semigroup $\left\{T_{t}\right\}$ satisfying (C.1)-(C.3), then $\bar{Y}_{0}=X$ and $A_{0}$ is closable. Let $A=\bar{A}_{0}$, then $R(\lambda)=R(\lambda ; A)$ for $\operatorname{Re}(\lambda)>\omega_{1}$. Furthermore, $Y=\bigcap_{n \geqq 1} D\left(A^{n}\right)$ is a core of $A^{k}$.

Proof. It is proved in Hille-Phillips [8; Theorem 10.3.4] that $Y_{0}$ is dense in $X_{0}$. Hence, $\overline{Y_{0} \cap \bar{X}_{0}}=X$ by (C.1). Let $A_{\eta}=\eta^{-1}\left[T_{\eta}-I\right]$ for $\eta>0$, $\operatorname{Re}(\lambda)>\omega_{1}$ and $x \in \Sigma$, then by using the relation (5.6) we see that $R(\lambda) x$ $=R_{0}(\lambda) x \in D\left(A_{0}\right)$ and $\left(\lambda-A_{0}\right) R_{0}(\lambda) x=x$. Since $A_{\eta} x \in \Sigma$, we have $R(\lambda) A_{\eta} x$ $=R_{0}(\lambda) A_{\eta} x=A_{\eta} R_{0}(\lambda) x=A_{\eta} R(\lambda) x$, and hence $A_{0} R(\lambda) x=R(\lambda) A_{0} x$ provided that $x \in D\left(A_{0}\right)$. Consequently, we obtain

$$
\left(\lambda-A_{0}\right) R(\lambda) x=R(\lambda)\left(\lambda-A_{0}\right) x=x,
$$

for $x \in D\left(A_{0}\right)$ and $\operatorname{Re}(\lambda)>\omega_{1}$. Now, assume that $x_{n} \in D\left(A_{0}\right), x_{n} \rightarrow 0$ and $A_{0} x_{n} \rightarrow y_{0}$, then by $(6.1)$ we see that $\lambda R(\lambda) x_{n}-R(\lambda) A_{0} x_{n}=x_{n}$ for $\lambda>\omega_{1}$ and $n$. Hence, letting $n \rightarrow \infty$ we have that $R(\lambda) y_{0}=0$ for $\lambda>\omega_{1}$. It then follows from (C.3) that $y_{0}=0$. This means that $A_{0}$ is closable. Let $A$ $=\bar{A}_{0}$, then for $x \in D(A)$ there is a sequence $\left\{x_{n}\right\} \subset D\left(A_{0}\right)$ with $x_{n} \rightarrow x$ and $A_{0} x_{n} \rightarrow A x$. Thus, if $\operatorname{Re}(\lambda)>\omega_{1}$, then (6.1) yields that $R(\lambda)(\lambda-A) x$ 
$=x$. Also, since $\overline{D\left(A_{0}\right)}=X$, for each $x \in X$ we can find a sequence $\left\{x_{n}\right\}$ $C D\left(A_{0}\right)$ with $x_{n} \rightarrow x$. Since $R(\lambda) x_{n} \in D\left(A_{0}\right)$ and $R(\lambda) x_{n} \rightarrow R(\lambda) x$, again (6.1) implies that $A_{0} R(\lambda) x_{n}=\lambda R(\lambda) x_{n}-x_{n} \rightarrow \lambda R(\lambda) x-x$. So that $A R(\lambda) x$ $=\lambda R(\lambda) x-x$. Therefore, it follows that $R(\lambda)=R(\lambda ; A)$. Since $\rho(A)>$ $\left\{\lambda \in \mathbf{R} ; \lambda>\omega_{1}\right\}$, the last assertion follows from Lemma 3.6. Q.E.D.

Definition 6.3. $A=\bar{A}_{0}$ is called the complete infinitesimal generator.

Definition 6.4. Let $\left\{T_{t}\right\}$ be a semigroup of bounded operators satisfying (C.1)-(C.3) and $A$ be its complete infinitesimal generator. Then $\left\{T_{t}\right\}$ is said to be of class $\left(C_{(k)}\right)$, if there is a $k \in \mathbb{Z}_{+}$such that $D\left(A^{k}\right) \subset \Sigma$.

Remark 6.5. The above definition is equivalent to the following: A semigroup $\left\{T_{t}\right\}$ is said to be of class $\left(C_{(k)}\right)$, if it has the properties (C.1) $-(\mathrm{C} .3)$ and

(C.4) $R\left(R\left(\lambda_{0}\right)^{k}\right) \subset \Sigma \quad$ for some $\lambda_{0}>\omega_{1}$.

Lemma 6.6. Let $\left\{T_{t}\right\}$ be a semigroup of class $\left(C_{(k)}\right)$ with the type $\omega_{0}$ and $A$ be the complete infinitesimal generator. Then $A \in \mathbb{S}_{2}\left(\omega_{1}, k\right)$ for some $\omega_{1}>\omega_{0}$ and condition $(F ; k)$ holds.

Proof. Since $R_{0}(\xi) x=R(\xi ; A) x$ for $\xi>\omega_{1}$ and $x \in \Sigma$ by Lemma 6.2, the assertion follows from Theorem 5.10.

Q.E.D.

Lemma 6.7. Let $k \in \mathbb{Z}_{+}$. Then an operator $A \in \mathfrak{S}(X)$ is the complete infinitesimal generator of at most one semigroup $\left\{T_{t} ; t>0\right\}$ of class $\left(C_{(k)}\right)$.

Proof. Suppose that $\left\{T_{t}\right\}$ and $\left\{\widetilde{T}_{t}\right\}$ satisfy (C.1)-(C.4) for $k$ and that $A$ be the complete infinitesimal generator of both semigroups. If $x \in D\left(A^{k}\right)$, then by Lemma $6.2, R(\xi ; A) x=\int_{0}^{\infty} e^{-\xi t} T_{t} x d t=\int_{0}^{\infty} e^{-\xi t} \widehat{T}_{t} x d t$ for $\xi$ sufficiently large. But, both $T_{t} x$ and $\widetilde{T}_{t} x$ are strongly continuous in $t>0$, and hence it follows from the unicity of Laplace transform that $T_{t} x=\widetilde{T}_{t} x$ for $t>0$. On the other hand, Lemma 6.2 yields that $\overline{D\left(A^{k}\right)}=X$, and so, $T_{t}=\widetilde{T}_{t}$ for $t>0$.

Q.E.D.

The next result gives a generation theorem of semigroups of class $\left(C_{(k)}\right)$.

Theorem 6.8. Let $A$ be a densely defined, closed operator belonging to $\mathbb{S}_{1}(\omega, k)$ for some $\omega \in \mathbb{R}$ and $k \in \mathbb{Z}_{+}$and satisfying $\left(F ; k^{\prime}\right)$ for some $k^{\prime} \in \mathbb{Z}_{+}$. Then $A$ is the complete infinitesimal generator of a unique semigroup $\left\{T_{t}\right\}$ of class $\left(C_{(k)}\right)$ such that 
(a) for $x \in D\left(A^{k}\right)$ and $t \geqq 0, T_{t} x=\lim _{h \rightarrow+0}(I-h A)^{-[t / h]} x$, and the convergence is uniform with respect to $t$ in every finite interval;

(b) for every $\varepsilon>0$ there is an $M_{\varepsilon}>0$ such that $\sup \left\{\left\|T_{t}\right\| ; \varepsilon \leqq t \leqq 1 / \varepsilon\right\}$ $\leqq M_{\varepsilon}$;

(c) for every $l \in \mathbf{Z}_{+}, A^{l} T_{t} x=T_{t} A^{l} x$ for $x \in D\left(A^{l}\right)$;

(d) for every $x \in X, T_{t} x$ is strongly continuous in $t>0 ; D\left(A^{k}\right) \subset \Sigma$; for $x \in D\left(A^{k+1}\right), T_{t} x-x=\int_{0}^{t} T_{s} A x d s=\int_{0}^{t} A T_{s} x d s$ for $t \geqq 0 ;$ and hence $D\left(A_{0}\right)$ $\supset D\left(A^{k+1}\right)$,

(e) if in particular, $k \geqq 1$, then for every $x \in D(A)$ and $t>0,(d / d t)$ $T_{t} x=A T_{t} x=T_{t} A x$.

Proof. Let $\left\{U_{t} ; t \geqq 0\right\} \subset \mathfrak{B}\left(D\left(A^{k}\right), X\right)$ be the one-parameter family obtained by Theorem 4.2. Then by Theorem 5.17 and Lemma 5.16, we obtain a semigroup of bounded operators $\left\{T_{t}\right\}$ having the properties (a) and (b). In order to show (c), we first prove that each $R(\mu ; A)$ commutes with $T_{t}, t>0$. For $x \in D\left(A^{k}\right)$ and $\lambda>\omega_{1}, R(\mu ; A) J_{\lambda}^{[t / \lambda]} x=J_{\lambda}^{[t / \lambda]} R(\mu ; A) x$. Hence, by (a) we have that $R(\mu ; A) U_{t} x=U_{t} R(\mu ; A) x$. But, since $\bar{U}_{t}=$ $T_{t} \in \mathfrak{B}(X)$ and $\left.\overline{D\left(A^{k}\right.}\right)=X$, we see that $R(\mu ; A) T_{t}=T_{t} R(\mu ; A)$ for $t>0$. Assume that $x \in D\left(A^{l}\right)$, then $x=R(\mu ; A)^{l} y$ for some $y \in X$. Hence, $T_{t} x$ $=T_{t} R(\mu ; A)^{l} y=R(\mu ; A)^{l} T_{t} y \in D\left(A^{l}\right) \quad$ and $\quad T_{t} A^{l} x=T_{t}[A R(\mu ; A)]^{l} y=$ $A^{l} T_{t} x$. Property (d) is the restated form of Theorem 4.2 (e). Next, we show (e). Let $\varepsilon>0$ and $\varepsilon<s<t<1 / \varepsilon$. Then $\left\|T_{\sigma}\right\| \leqq M_{\varepsilon}$ for $\varepsilon \leqq \sigma \leqq 1 / \varepsilon$. Since $D\left(A^{k+1}\right)$ is a core of $A$, it follows that for each $x \in D(A)$ there is a sequence $\left\{x_{n}\right\} \subset D\left(A^{k+1}\right)$ with $x_{n} \rightarrow x$ and $A x_{n} \rightarrow A x$. Hence, $U_{\sigma} A x_{n} \rightarrow$ $T_{\sigma} A x$ boundedly for $\varepsilon \leqq \sigma \leqq 1 / \varepsilon$. Since $T_{t} x_{n}-T_{s} x_{n}=\int_{s}^{t} T_{\sigma} A x_{n} d \sigma$, we obtain $T_{t} x-T_{s} x=\int_{s}^{t} T_{\sigma} A x d \sigma$.

Now, we demonstrate that the semigroup $\left\{T_{t}\right\}$ constructed belongs to class $\left(C_{(k)}\right)$. First, $D\left(A^{k}\right) \subset \Sigma$ by (d); and so $\bar{X}_{0}=X$. Let $\omega_{1}>\max \left\{\omega, \omega_{0}\right\}$ and $R_{0}(\lambda)$ be the operator defined by the formula (5.3) for this semigroup. Then $D\left(R_{0}(\lambda)\right) \supset \Sigma$ for $\operatorname{Re}(\lambda)>\omega_{1}$. Also, from Theorem 4.6 it follows that $R(\lambda ; A) x=R_{0}(\lambda) x$ for $x \in D\left(A^{k}\right)$ and $\operatorname{Re}(\lambda)>\omega_{1}$. Now, for every $x \in X_{0}$ there are $t_{0}>0$ and $y \in X$ such that $x=T_{t_{0}} y$. Since $\overline{D\left(A^{k}\right)}=X$, there exists a sequence $\left\{y_{n}\right\} \subset D\left(A^{k}\right)$ with $y=\lim y_{n}$ and $T_{t_{0}} y_{n} \in D\left(A^{k}\right)$. Therefore, we have 


$$
\begin{aligned}
& R(\lambda ; A) x=R(\lambda ; A) T_{t_{0}} y=\lim R(\lambda ; A) T_{t_{0}} y_{n}=\lim R_{0}(\lambda) T_{t_{0}} y_{n} \\
& \quad=R_{0}(\lambda) T_{t_{0}} y=R_{0}(\lambda) x
\end{aligned}
$$

note that $R_{0}(\lambda) T_{t_{0}} \in \mathfrak{B}(X)$ for $\operatorname{Re}(\lambda)>\omega_{1}$ and $t_{0}>0$. This means that $\left\{T_{t}\right\}$ satisfies (C.2). (C.3) is clear, since $R(\lambda ; A)$ is one-to-one. Finally, the uniqueness of the semigroup follows from Lemma 6.7. Q.E.D.

In view of this theorem we obtain a criterion for the existence of the solution of $A C P_{2}$.

Corollary 6.9. Let $A \in \mathbb{S}_{1}(\omega, k)$ for some $\omega \in \mathbf{R}$ and $k \in \mathbf{Z}_{+} \backslash\{0\}$. If $A$ satisfies either of $\left(F ; k^{\prime}\right)$ and $\left(C ; k^{\prime}\right)$ for some $k^{\prime} \geqq k$, then for every $x \in D\left(A^{k}\right)$, there is a unique solution $u(t ; x)$ of $A C P_{2}$ for $A$ with $\lim _{t \rightarrow+0}$ $u(t ; x)=x$.

Proof. In view of the properties (d) and (e) of Theorem 6.8, for each $x \in D\left(A^{k}\right), u(t ; x)=T_{t} x$ becomes a solution of $A C P_{2}$ for $A$ with $\lim _{t \rightarrow+0}$ $u(t ; x)=x$. To prove the uniqueness, we let $u(t)$ be another solution of $A C P_{2}$ satisfying $\lim _{t \rightarrow+0} u(t)=x$ and then put $v(t)=T_{t} x-u(t), t>0$, note that $\lim _{t \rightarrow+0} v(t)=0$. Let $\lambda_{0} \in \rho(A)$, then $R\left(\lambda_{0} ; A\right)^{k} v(s) \in D\left(A^{k+1}\right)$; and hence $\stackrel{t \rightarrow+0}{\text { by }}$ a similar way to the proof of Lemma 2.4 we have that $(\partial / \partial s)$ $T_{t-s} R\left(\lambda_{0} ; A\right)^{k} v(s)=0$ for $0<s<t$. Therefore, $0=\int_{0}^{t}(\partial / \partial s) T_{t-s} R\left(\lambda_{0} ; A\right)^{k} v$ $(s) d s=\lim _{\varepsilon \rightarrow+0}\left[T_{t-s} R\left(\lambda_{0} ; A\right)^{k} v(s)\right]_{\varepsilon}^{t-\varepsilon}=R\left(\lambda_{0} ; A\right)^{k} v(t)$ for $t>0$. This means that $v(t) \equiv 0$ for $t>0$.

Q.E.D.

Remark 6.10. Let $\left\{T_{t}\right\}$ be the semigroup constructed in Theorem 6.8. Then $\left\{T_{t} \mid D\left(A^{k}\right)\left(=U_{t}\right) ; t>0\right\}$ forms a semigroup of bounded operators on the linear manifold $D\left(A^{k}\right)$. This semigroup is strongly continuous at $t>0$. Also, Corollary 6.9 states that $T_{t} \mid D\left(A^{k}\right)$ is a solution operator of $A C P_{2}$ for $A$.

Remark 6.11. Let $\left\{T_{t}\right\}$ be the semigroup obtained by Theorem 6.8 for an $A \in \mathscr{B S}_{1}(\omega, k), k \geqq 1$. Take an $x \in D\left(A^{k}\right)$, then by Theorem $6.8(\mathrm{e})$, $(d / d t) e^{-\lambda t} T_{t} x=-e^{-\lambda t} T_{t}(\lambda-A) x$ for $t>0$ and $\lambda \in \mathbb{C}$. Let $\omega_{0}$ be the type of $\left\{T_{t}\right\}$ and $\omega_{1}>\max \left\{\omega, \omega_{0}\right\}$. Then for every $\lambda \in \mathbf{C}$ with $\operatorname{Re}(\lambda)>\omega_{1}$,

$$
e^{-\lambda \delta} T_{\delta} x=\int_{\delta}^{\infty} e^{-\lambda t} T_{t}(\lambda-A) x d t
$$

Since $e^{-\lambda \delta} T_{\delta} x \rightarrow x$ as $\delta \rightarrow+0$ by Theorem 6.8 (d), the improper integral 
$\int_{0}^{\infty} e^{-\lambda t} T_{t}(\lambda-A) x d t=\lim _{\delta \rightarrow+0} \int_{\delta}^{\infty} e^{-\lambda t} T_{t}(\lambda-A) x d t$ converges and is equal to $x$. Consequently, we obtain the following relation:

$$
R(\lambda ; A) z=\int_{0}^{\infty} e^{-\lambda t} T_{t} z d t, \quad z \in D\left(A^{k-1}\right) .
$$

Now, we state a characterization of semigroups of class $\left(C_{(k)}\right)$.

Theorem 6.12. An operator $A$ in $X$ is the complete infinitesimal generator of a semigroup $\left\{T_{t}\right\}$ of class $\left(C_{(k)}\right)$ if and only if $\overline{D(A)}=X$, $A \in \mathrm{SS}_{1}(\omega, k)$ and $(F ; k)$ is satisfied.

Proof. The necessity is verified in Lemma 6.6 and the sufficiency follows from Theorem 6.8.

Q.E.D.

Next, we consider an example which shows that class $\left(C_{(k)}\right)$ is properly contained in class $\left(C_{(k+1)}\right)$.

Example 6.13. In a similar way to Example 4.10, let us consider an unbounded operator $P$ of multiplication, defined in $L_{2}=\overbrace{L_{2}(\mathbf{R}) \times \cdots \times L_{2}(\mathbf{R})}^{k+1}$ by (4.8), such that $P(\xi)$ is a $(k+1) \times(k+1)$-matrix of the form (4.10) with

$$
p=p(\xi)=-\xi^{2 \nu}+i \xi^{d} \text { and } q=q(\xi)=\xi^{r}, \quad \xi \in \mathbf{R},
$$

where $\nu, d$ and $r$ are positive integers. H. Sunouchi treated in [22] this example for the case in which $k=1, \nu=1, d=4$ and $0 \leqq r \leqq 4$ and he proved that if $r \leqq 2$ then the resultant semigroup is of class $\left(C_{0}\right)$ and that if $r=3$ or 4 then the resultant semigroup is of class $(0, A)$.

Since the eigen-values of each matrix $P(\xi)$ are equal to $-\xi^{2 \nu}+i \xi^{d}$, operator $P$ has the (thin) spectrum $\sigma(P)=\left\{-\xi^{2 \nu}+i \xi^{d} ; \xi \in \mathbf{R}\right\}$. In view of (4.11), (4.12) and (4.13), if $d(k+1) \geqq r k$ and $\operatorname{Re}(\lambda)>0,(\lambda-P(\xi))^{-1}, \xi \in \mathbf{R}$, define a bounded operator $(\lambda-P)^{-1}$ on $L_{2}$ which is the resolvent of $P$ at $\lambda$. Thus, in this case, we assume that

$$
2 \nu<d<r \leqq k^{-1}(k+1) d .
$$

First, we show that for each $t>0, e^{t P(\xi)}, \xi \in \mathbf{R}$, define a bounded operator $e^{t P}$ on $L_{2}$. Let $\phi=\left(\phi_{j}\right) \in L_{2}$. Then, as stated in Example 4.10, the $j$-th element of $e^{t P} \phi$ was $\left[e^{t P} \phi\right]_{j}=e^{p t} \sum_{l=0}^{k+1-j}(l !)^{-1} t^{l} q^{l} \phi_{j+l}$. 
On the other hand, for every $\varepsilon>0$ there is a positive constant $N_{\varepsilon}$ such that $\sup _{\xi} e^{t \operatorname{Re}(p(\xi))}|q(\xi)|^{l} \leqq \sup \exp _{\xi}\left(-\varepsilon \xi^{2 \nu}\right)|\xi|^{r l} \leqq N_{\varepsilon}$ for $0 \leqq l \leqq k$ and $t \geqq \varepsilon$. Hence,

$$
\left\|\left[e^{t P} \phi\right]_{j}\right\| \leqq \sum_{l=1}^{k+1-j}(l !)^{-1} t^{l}\left\|e^{p t} q^{l} \phi_{j+l}\right\| \leqq N_{\varepsilon} e^{t} \max _{l}\left\|\phi_{j+l}\right\| \leqq N_{\varepsilon} e^{t}\|\phi\| .
$$

Since $\left\|e^{t P} \phi\right\|^{2}=\sum_{j=0}^{k+1}\left\|\left[e^{t P} \phi\right]_{j}\right\|^{2} \leqq(k+1) N_{\varepsilon}^{2} e^{2 t}\|\phi\|^{2}$, it follows that for every $\varepsilon>0$ there exists a positive number $M_{\varepsilon}$ such that

$$
\left\|e^{t P} \phi\right\| \leqq M_{\varepsilon}\|\phi\| \quad \text { for } t \in[\varepsilon, 1 / \varepsilon] \text { and } \phi \in L_{2}
$$

Thus $e^{t P}$ defines a bounded operator on $L_{2}$ for each $t>0$. It is easy to see that $e^{t P} e^{s P}=e^{(t+s) P}$ for $t, s>0$ and also that $e^{t P} \phi$ is weakly measurable with respect to $t>0$ for $\phi \in L_{2}$. Since $L_{2}$ is a separable Hilbert space, $\left\{e^{t P} ; t>0\right\}$ is strongly measurable and hence it forms a semigroup of bounded operators on $L_{2}$.

We can show that $e^{t P} \phi$ is strongly continuous in $t \geqq 0$ for every $\phi \in$ $D\left(P^{k+1}\right)$. In fact, we observed in Example 4.10 that if $\phi \in D\left(P^{k+1}\right)$ then $q^{l} \phi_{j+l} \in L_{2}(R)$ for $1 \leqq j \leqq k+1$ and $0 \leqq l \leqq k+1-j$, and so, $\left\|e^{p t} q^{l} \phi_{j+l}\right\| \leqq$ $\left\|q^{l} \phi_{j+l}\right\|$. Also, it is easy to see that $\lim _{t \rightarrow+0}\left\|e^{p t} \phi_{j}-\phi_{j}\right\|=0$. Hence, $\|\left[e^{t P} \phi\right]_{j}$ $-\phi_{j}\|\leqq\| e^{p t} \phi_{j}-\phi_{j}\left\|+\sum_{l=1}^{k+1-j}(l !)^{-1} t^{l}\right\| q^{l} \phi_{j+l} \| \rightarrow 0$ as $t \rightarrow+0$ for $1 \leqq j \leqq k+1$. This means that $e^{t P} \phi$ is strongly continuous in $t \geqq 0$ for every $\phi \in D\left(P^{k+1}\right)$. Therefore, if $\Sigma$ denotes the continuity set of $\left\{e^{t P}\right\}$, then $D\left(P^{k+1}\right) \subset \Sigma$. This also implies that $X_{0}=\bigcup_{t>0} e^{t P}\left[L_{2}\right]$ is dense in $X$. Now, let $\omega_{0}$ be the type of the semigroup $\left\{e^{t P}\right\}$ and let $\omega_{1}>\max \left\{0, \omega_{0}\right\}$. If $\phi \in \Sigma$ and $\operatorname{Re}(\lambda)$ $>\omega_{1}$, then $\int_{0}^{\infty} e^{-\lambda t} e^{t P} \phi d t$ makes sense. Hence, we see applying Fubini's theorem that

$$
\left\langle\int_{0}^{\infty} e^{-\lambda t} e^{t P} \phi d t, \phi\right\rangle=\int_{0}^{\infty} e^{-\lambda t}\left\langle e^{t P} \phi, \phi>d t=\left\langle(\lambda-P)^{-1} \phi, \phi\right\rangle .\right.
$$

This means that $(\lambda-P)^{-1} \phi=\int_{0}^{\infty} e^{-\lambda t} e^{t P} \phi d t$ for $\phi \in \Sigma$ and $\operatorname{Re}(\lambda)>\omega_{1}$. Consequently, we can say that $\left\{e^{t P}\right\}$ is a semigroup of class $\left(C_{(k+1)}\right)$. Let $\left\{T_{t} ; t>0\right\}$ be the one-parameter family of bounded operators on $L_{2}$ which are defined by the inverse Fourier transforms of $e^{t P} \phi, t>0$. The above-mentioned states that $\left\{T_{t}\right\}$ forms a semigroup of class $\left(C_{(k+1)}\right)$.

Next, we demonstrate that the semigroup $\left\{T_{t}\right\}$ defined above is not 
of class $\left(C_{(k)}\right)$; this fact proves that $\left(C_{(k)}\right) \varsubsetneqq\left(C_{(k+1)}\right)$. Since it is well-known that $\left(C_{(0)}\right)=\left(C_{0}\right) \varsubsetneqq(0, A) \subset\left(C_{(1)}\right)$, let us assume that $k \geqq 1$.

Let $\phi$ be the element of $L_{2}$ of the form (4.19). Then, under the restriction (6.4), we can proceed with the same argument as in Example 4.10 and can prove that $\phi \in D\left(P^{k}\right) \backslash D\left(P^{k+1}\right)$ provided that

$$
r-d=a+b, d-2 \nu=b+c, \text { and } a, b, c \geqq 1 .
$$

Indeed, what is needed is the degree of $p(\xi)(=d$ under (6.4)) and $\nu$ does not exert any influence upon the proof as long as (6.4) and the restrictions on $r-d$ and $d-2 \nu$ are assumed. (Note that, in this case,

$$
\begin{aligned}
{\left[P^{k} \phi\right]_{1} } & =k p q^{k-1} \phi_{k}+q^{k} \phi_{k+1}=k q^{k-1}(1+|\xi|)^{b-r k}\left(p\left(i \xi^{r-d}+1\right)+q\right) \\
& =k q^{k-1}(1+|\xi|)^{b-r k}\left(p+i \xi^{2 \nu+r-d}\right)
\end{aligned}
$$

and that $\operatorname{deg}\left(q^{k-1} i \xi^{2 \nu+r-d}\right)=r k-b-c$. $)$ We then show that if furthermore $b \geqq 2 \nu k$ then $e^{t P} \phi$ is discontinuous at $t=0$. (Hence, $d \geqq 2 \nu(k+1)+c$.)

By virtue of the relation (4.20), we obtain

$$
\left[e^{t P} \phi\right]_{1}=k !^{-1} t^{k} e^{p t}(q+i)^{k} \phi_{k+1}+(k+1) !^{-1} t^{k-1} e^{p t}\left\{q^{k-1} \phi_{k}+k^{-1} t Q \phi_{k+1}\right\}
$$

where $Q=q^{k}-(q+i)^{k}$. Since $\operatorname{deg}(Q)=\operatorname{deg}\left(q^{k-1}\right), Q \phi_{k+1} \in L_{2}(R)$. Hence,

$$
\begin{aligned}
& \left\|e^{t P} \phi\right\|^{2} \geqq\left\|\left[e^{t P} \phi\right]_{1}\right\|_{2} \\
\geqq & 2^{-1}\left(t^{k} / k !\right)^{2} \int_{-\infty}^{\infty}(1+|\xi|)^{2 b-2 r k}\left|\xi^{r}+i\right|^{2 k} \exp \left(-2 \xi^{2 \nu} t\right) d \xi \\
& -\left(t^{k-1} /(k-1) !\right)^{2}\left\|q^{k-1} \phi_{k}+k^{-1} t Q \phi_{k+1}\right\|^{2} .
\end{aligned}
$$

Since a positive number $C$ can be found such that $(1+|\xi|)^{-2 r k}\left|\xi^{r}+i\right|^{2 k}$ $\geqq C$, the first term of the right hand side is greater than

$$
C\left(t^{k} / k !\right)^{2} \int_{-\infty}^{\infty} \xi^{2 b} \exp \left(-2 \xi^{2 \nu} t\right) d \xi
$$

Now, setting $2 \xi^{2 \nu} t=\sigma^{2 \nu}$, we have that $\xi^{2}=(2 t)^{-1 / \nu} \sigma^{2}$ and $d \xi=(2 t)^{-1 / 2 \nu} d \sigma$, and hence the integral is equal to 


$$
2 C k^{-2} t^{2 k-b / \nu-1 / 2 \nu} \int_{0}^{\infty} \sigma^{2 b} e^{-\sigma^{2}} d \sigma .
$$

This means that $e^{t P} \phi$ blows up at $t=0$, if $b \geqq 2 k \nu$. Therefore, the $\left\{T_{t}\right\}$ is not of class $\left(C_{(k)}\right)$.

In (6.4) we assumed that $2 \nu<d$. If $d(k+1) \geqq r k$ and $2 \nu \geqq d$, then the spectrum $\sigma(P)$ is contained in a sector and the semigroup $\left\{e^{t P}\right\}$ can be extended to a holomorphic semigroup. In this case, $D\left(P^{k}\right)$ is contained in the continuity set $\Sigma$. In fact, in this case,

$$
\begin{aligned}
e^{t P} & =e^{p t} e^{t(P-p I)}=e^{p t} \sum_{l=0}^{k}(l !)^{-1} t^{l}(P-p)^{l} \\
& =e^{p t} \sum_{l=0}^{k} \sum_{j=0}^{l}(l-j) !^{-1} t^{l-j}(-p)^{l-j}(j !)^{-1} t^{j} P^{j} \\
& =e^{p t} \sum_{j=0}^{k}\left[\sum_{l=0}^{k-j}(l !)^{-1} t^{l}(-p)^{l}\right](j !)^{-1} t^{j} P^{j}
\end{aligned}
$$

Since $2 \nu \geqq d$, we see that $\max _{j} \sup _{\xi, t}\left|e^{p t} \sum_{l=0}^{k-j}(l !)^{-1}(-p t)^{l}\right|=N<+\infty$; hence, for each $\phi \in D\left(P^{k}\right)$, we obtain

$$
\left\|e^{t P} \phi\right\| \leqq \sum_{j=0}^{k} N(j !)^{-1} t^{j}\left\|P^{j} \phi\right\| \leqq N e^{t}\|\phi\|_{k}
$$

Hence, it follows that $\lim _{t \rightarrow+0}\left\|e^{t P} \phi-\phi\right\|=0$ for $\phi \in D\left(P^{k}\right)$.

Finally, we discuss some generation theorems (of Feller type) for $(A)$-, $(0, A)-,(1, A)$ - and $\left(C_{0}\right)$-semigroups.

First, the following type of characterization of $(A)$-semigroups is stated in Hille-Phillips [8; Theorem 12.5.1].

Theorem 6.14. An operator $A$ in $X$ is the complete infinitesimal generator of an $(A)$-semigroup $\left\{T_{t}\right\}$ if and only if $A \in \mathbb{S}_{3}(\omega, 0)$ for some $\omega \in R, s-\lim _{\xi \rightarrow+\infty} \xi R(\xi ; A)=I$, and condition $(F ; k)$ is satisfied for some $k \in \mathbf{Z}_{+}$. Furthermore, in this case, $\left\{T_{t}\right\}$ is of class $\left(C_{(2)}\right)$.

Proof. Assume that $A \in \mathcal{S S}_{3}(\omega, 0), s-\lim \xi R(\xi ; A)=I$, and that $(F ; k)$ holds. Then by Theorems 4.7 and 5.17 and then by Theorem 6.8, $A$ is the complete infinitesimal generator of a semigroup $\left\{T_{t}\right\}$ of class $\left(C_{(2)}\right)$. Since $R(\lambda ; A) x=\int_{0}^{\infty} e^{-\lambda t} T_{t} x d t$ for $\operatorname{Re}(\lambda)>\omega_{1}$ and $x \in X_{0}$ and for some $\omega_{1}>$ $\max \left\{\omega, \omega_{0}\right\}$ by Lemma 6.2 and since sup $\left\{\|R(\lambda ; A)\| ; \operatorname{Re}(\lambda)>\omega_{1}\right\}<+\infty$ and $s-\lim \xi R(\xi ; A)=I$, it follows that $\left\{T_{t}\right\}$ is an $(A)$-semigroup. Conversely, 
assume that $A$ is the complete infinitesimal generator of an $(A)$-semigroup $\left\{T_{t}\right\}$ with the type $\omega_{0}$. Then $A \in \mathscr{S}_{3}\left(\omega_{1}, 0\right)$ for some $\omega_{1}>\omega_{0}$ by Remark 4.8. Hence, it follows from Theorem 4.7 and 4.6 that $D\left(A^{2}\right) \subset \Sigma$. This means that $\left\{T_{t}\right\}$ is of class $\left(C_{(2)}\right)$; and hence $(F ; 2)$ is satisfied. Let $R(\lambda), \operatorname{Re}(\lambda)>\omega_{1}$, be the operators given in condition (A.1) which is stated in Section 1 , then $R(\lambda)=R(\lambda ; A)$ for $\operatorname{Re}(\lambda)>\omega_{1}$ by Lemma 6.2 , and so $s-\lim _{\xi \rightarrow+\infty} \xi R(\xi ; A)=I$.

Q.E.D.

Theorem 6.15. An operator $A$ in $X$ is the complete infinitesimal generator of a $(0, A)$-semigroup if and only if $A \in \mathbb{S}_{1}(\omega, 1), s-\lim \xi R(\xi ; A)$ $=I$, and

(F) $)_{0}$ for every $x \in X$, there is a measurable and locally summable function $f(t, x)$ on $(0, \infty)$ such that

(a) $\varlimsup_{t_{n} \uparrow t} f\left(t_{n}, x\right) \leqq f(t, x)$ and $\varlimsup_{t_{n} \downarrow t} f\left(t_{n}, x\right) \leqq f(t, x)$,

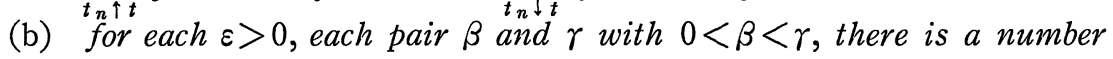
$\xi_{0} \equiv \xi_{0}(x ; \beta, \gamma, \varepsilon)$ with

$$
\left\|\xi^{n} R(\xi ; A)^{n} x\right\| \leqq f(n / \xi, x)+\varepsilon, \text { for } \beta \leqq n / \xi \leqq \gamma \text { and } \xi>\xi_{0} .
$$

Furthermore, in this case, $\left\|T_{t} x\right\| \leqq f(t, x)$ for $t>0$ and $\left\{T_{t}\right\}$ is of class $\left(C_{(1)}\right)$.

Proof. Assume that $A \in \mathbb{S}_{1}(\omega, 1)$ and that $(\mathrm{F})_{0}$ is satisfied. Let $x \in$ $X, \varepsilon>0$, and $0<\beta<\gamma$. Since every function satisfying $(\mathrm{F})_{0}-(\mathrm{a})$ is upper bounded on every compact interval, $N(x ; \beta, \gamma) \equiv \sup \{f(s, x) ; \beta \leqq s \leqq \gamma\}<$ $+\infty$. Hence, by $(\mathrm{F})_{0}-(\mathrm{b})$ there is a $\xi_{0} \equiv \xi_{0}(x ; \beta, \gamma, \varepsilon)$ such that $\| \xi^{n} R$ $(\xi ; A)^{n} x \| \leqq N(x ; \beta, \gamma)+\varepsilon$ for $\beta \leqq n / \xi \leqq \gamma$ and $\xi>\xi_{0}$. On the other hand, let $\omega_{1}>\max \{0, \omega\}$, then by the assumption there exists a positive number $K$ such that $\|\xi R(\xi ; A)\| \leqq K$ for $\xi>\omega_{1}$. Hence, $\left\|\xi^{n} R(\xi ; A)^{n} x\right\| \leqq K^{\left[\xi_{0} \gamma\right]+1}$ $\|x\|$ for $\beta \leqq n / \xi \leqq \gamma$ and $\omega_{1}<\xi<\xi_{0}$. Consequently, it follows that $\left\{\xi^{n} R(\xi\right.$; $\left.A)^{n} x ; \beta \leqq n / \xi \leqq \gamma, \xi>\omega_{1}\right\}$ is bounded for each $x \in X$. Thus, by the resonance theorem a positive number $M_{\beta . \gamma}$ can be found such that

$$
|| \xi^{n} R(\xi ; A)^{n} \| \leqq M_{\beta . \gamma} \quad \text { for } \beta \leqq n / \xi \leqq \gamma \text { and } \xi>\omega_{1} .
$$

Since (6.5) is nothing but condition $(F ; 0)$, it follows from Theorem 6.8 
(a) that

$$
T_{t}=s-\lim _{h \rightarrow+0}(I-h A)^{-[t / h]}, \quad t>0,
$$

exist uniformly with respect to $t$ in each compact interval of $(0, \infty)$; the family $\left\{T_{t}\right\}$ forms a semigroup of class $\left(C_{(1)}\right)$. Now, fix an $x \in X$ and a $t>0$. Then, for any $\varepsilon>0$ and any pair $\beta$ and $\gamma$ with $0<\beta<t<\gamma$, there is an $h_{0}$ sufficiently small such that $\beta \leqq[t / h] h \leqq t \leqq[t / h] h+h \leqq \gamma$ and $\left\|(I-h A)^{-[t / h]} x\right\| \leqq f([t / h] h ; x)+\varepsilon$ provided $h \in\left(0, h_{0}\right]$. Since $[t / h] h \hat{\uparrow} t$ or $[t / h] h+h \downarrow t,(\mathrm{~F})_{0}-(\mathrm{a})$ yields that $\left\|T_{t} x\right\| \leqq \varlimsup_{h \downarrow 0} f([t / h] h, x)+\varepsilon \leqq f(t, x)$ $+\varepsilon$ or $\left\|T_{t} x\right\| \leqq \varlimsup_{h \downarrow 0} f([t / h] h+h, x)+\varepsilon \leqq f(t, x)+\varepsilon$. Hence, by virtue of Remark 6.11 and $(F ; 0), R(\lambda ; A) x=\int_{0}^{\infty} e^{-\lambda t} T_{t} x d t$ for $x \in X$ and $\operatorname{Re}(\lambda)>$ $\omega_{1}>\max \left\{0, \omega, \omega_{0}\right\}$, where $\omega_{0}$ is the type of $\left\{T_{t}\right\}$. Therefore, $\xi \int_{0}^{\infty} e^{-\xi t} T_{t} x d t$ $\rightarrow x$ as $\xi \rightarrow+\infty$, and so $\left\{T_{t}\right\}$ is of class $(0, A)$ and $A$ is its complete infinitesimal generator. Conversely, let $A$ be the complete infinitesimal generator of a $(0, A)$-semigroup with the type $\omega_{0}$. Then $A \in \mathbb{S}_{2}\left(\omega_{0}, 1\right)$ by Remark 4.8. We then show that $(\mathrm{F})_{0}$ holds. By Hille-Phillips [8; Theorem 11.6.6, p. 352], we have the representation (6.6). Thus it is easy to see using the resonance theorem that $\sup \left\{\left\|\xi^{n} R(\xi ; A)^{n}\right\| ; \xi>\omega_{0}, \varepsilon \leqq n / \xi \leqq\right.$ $1 / \varepsilon\}<+\infty$ for each $\varepsilon>0$. So, it follows that for any convergent sequence $\left\{n_{j} / \xi_{j}\right\}$ such that $\xi_{j} \rightarrow+\infty$ and $n_{j} / \xi_{j} \rightarrow t>0$,

$$
T_{t}=s-\lim _{j \rightarrow+\infty}\left(I-\xi_{j}^{-1} A\right)^{-n} .
$$

We can now obtain $(\mathrm{F})_{0}$ by setting $f(t, x)=\left\|T_{t} x\right\|$ for $t>0$ and $x \in X$ and by employing the convergence (6.7).

Q.E.D.

Also, by a similar way to the proof of Theorem 6.15 , we obtain

Theorem 6.16. An operator $A$ in $X$ is the complete infinitesimal generator of $a(1, A)$-semigroup if and only if $A \in \mathbb{S}_{1}(\omega, 1), s-\lim _{\xi \rightarrow+\infty} \xi R(\xi ; A)$ $=I$, and

$(\mathrm{F})_{1}$ there is a measurable and locally summable function $f(t)$ on $(0, \infty)$ such that

(a) $\varlimsup_{t_{n} \uparrow t} f\left(t_{n}\right) \leqq f(t)$ and $\varlimsup_{t_{n} \downarrow t} f\left(t_{n}\right) \leqq f(t)$,

(b) for each $\varepsilon>0$, each pair $\beta$ and $\gamma$ with $0<\beta<\gamma$, there is a number 
$\xi_{0} \equiv \xi_{0}(x ; \beta, \gamma, \varepsilon)$ with

$$
\left\|\xi^{n} R(\xi ; A)^{n} x\right\|_{\leqq}(n / \xi)\|x\|+\varepsilon \quad \text { for } \beta \leqq n / \xi \leqq \gamma \text { and } \xi>\xi_{0} .
$$

Furthermore, in this case, $\left\|T_{t}\right\| \leqq f(t)$ for $t>0$ and $\left\{T_{t}\right\}$ is of class $\left(C_{(1)}\right)$. Finally, we state the Hille-Yosida-Miyadara-Phillips theorem in the following form:

Theorem 6.17. An operator $A$ in $X$ is the infinitesimal generator of a $\left(C_{0}\right)$-semigroup if and only if $A \in \mathbb{S}_{2}(\omega, 0)$ for some $\omega \in \mathbf{R}$.

\section{References}

[1] Chazarain, J., Problèmes de Cauchy au sens des distributions vectorielles et applications, C. R. Acad. Sci. Paris, 266 (1968), 10-13.

[2] Crandall M. and T. Liggett, Generation of semigroups of nonlinear transformations on general Banach spaces, Amer. J. Math. 93 (2) (1971), 265-298.

[3] Da Prato, G., Semigruppi regolarizzabili, Ricerche Mat. 15 (1966), 223-248.

[4] — Problèmes au bord de type mixte pour des équations paraboliques ou hyperboliques, preprint.

[5] Dunford N. and J. Schwartz, Linear Operators, part I: General Theory, Interscience, New York, 1958.

[6] Feller, W., On the generation of unbounded semigroups of bounded linear operators, Ann. of Math. (2) 58 (1953), 166-174.

[7] Fujiwara, D., A characterization of exponential distribution semigroups, J. Math. Soc. Japan, 18 (1966), 267-274.

[8] Hille E. and R.S. Phillips, Functional analysis and semi-groups, Amer. Math. Soc. Colloq. Publ. 31 (1957).

[9] Kato, T., Perturbation Theory for Linear Operators, Springer, 1966.

[10] Kōmura, T., Semigroups of operators in a locally convex space, J. Func. Anal. 2 (1968), 258-296.

[11] Krein, S.G., Linear Differential Equations in Banach Spaces (in Russian), Nauka, 1967.

[12] Lions, J., Les semi-groups distributions, Portugal. Math. 19 (1960), 141-164.

[13] Lax, P.D., Difference approximation to solutions of linear differential equations - An operator theoretic approach, Proc. Symp. on Partial Diff. Eqs., Berkeley, 1955.

[14] Miyadera, I., On the generation of strongly ergodic semi-groups of operators, II, Tôhoku Math. J. 6 (1954), 231-242.

[15] - Semigroups of operators in Frechet space and applications to partial differential equations, Tôhoku Math. J. 11 (1959), 162-183.

[16] Oharu, S., On the convergence of semigroups of operators, Proc. Japan Acad. 42 (8) (1966), 880-884.

[17] - On the generation of semigroups of nonlinear contractions, J. Math. Soc. Japan, 22 (1970), 526-550.

[18] Oharu S. and H. Sunouchi, On the convergence of semigroups of linear opera- 
tors, J. Func. Anal. 6 (1970), 292-304.

[19] Peetre, J., Sur la théorie des semi-groupes distributions, Séminaire sur les Équations au Derivées Partielles, Collège de France (1963-1964).

[20] Phillips, R. S., A note on the abstract Cauchy problem, Proc. Nat. Acad. Sci. U.S.A. 40 (1954), 244-248.

[21] Richtmyer, R. and K. Morton, Difference Methods for Initial-Value Problems, 2nd ed., Interscience, New York, 1967.

[22] Sunouchi, H., Convergence of semi-discrete difference schemes of Cauchy problems, Tôhoku Math. J. 22 (1970), 394-408.

[23] Thomée, V., Stability theory for partial differential operators, SIAM Review, 11 (1969), 152-195.

[24] Ushijima, T., Some properties of regular distribution semigroups, Proc. Japan Acad. 45 (1969), 224-227.

[25] — On the strong continuity of distribution semi-groups, J. Fac. Sci. Univ. Tokyo, Sect. I, 17 (1670), 363-372.

[26] Yosida, K., Functional Analysis, Springer, Berlin, 1965.

[27] - An operator-theoretical integration of the wave equation, J. Math. Soc. Japan, 8 (1956), 79-92.

Notes added in proof: 1. Mr. Konishi has called the attention of the author to a paper by M. Sova, "Problème de Cauchy pour équations hyperboliques opérationnelles à coefficients constants non-bornes", Ann. Scuola Norm. Sup. Pisa, 22 (1968), 67-100, which contains, among others, similar results to Lemmas 2.4 and 2.7 and Theorem 4.3.

2. In Theorem 5.4 we gave two sufficient conditions for a linear operator in $X$ to be the infinitesimal generator of an R.D.S.G. But, it can be proved that those are also necessary conditions. Hence, two kinds of characterizations of R.D.S.G. are obtained and the result gives a straightforward generalization of Theorem 5.5. 